\title{
OVERVIEW ON THE CHRYSOMELOIDEA SUPERFAMILY (COLEOPTERA: CERAMBYCIDAE, ORSODACNIDAE, CHRYSOMELIDAE) IN DOBROGEA (ROMANIA)
}

\author{
SANDA MAICAN, RODICA SERAFIM
}

\begin{abstract}
The study represents a synthesis about the distribution of the species from superfamily Chrysomeloidea in Dobruja (Dobrogea) region (South-eastern Romania). 407 species from 132 genera and 15 subfamilies are recorded, based on the published data and on the study of material preserved in the collections of the "Grigore Antipa" National Museum of Natural History (Bucharest) and the Institute of Biology Bucharest of the Romanian Academy. The species Lilioceris lilii (Scopoli), Oulema erichsonii (Suffrian), Euluperus cyaneus (Joannis) and Altica quercetorum quercetorum Foudras are mentioned for the first time in fauna of Dobrogea. Some endemic species are highlighted: Vadonia hirsuta (K. Daniel \& J. Daniel), Dorcadion equestre transsilvanicum Ganglbauer, D. gashtarovi Sama, Dascălu \& Pesarini, D. axillare Küster and Brachyta balcanica Hampe. Rosalia alpina alpina Linnaeus, Morimus asper funereus Mulsant and Cerambyx cerdo cerdo Linnaeus are protected species, included in the annexes of the Council Directive 92/43/EEC on the conservation of natural habitats and wild fauna and flora. Also, Brachyta balcanica Hampe, Pedostrangalia verticalis Germar and Neodorcadion exornatum (Frivaldsky von Frivald) are species of national interest requiring strict protection, listed in the Government Emergency Ordinance no. 57/2007 on the regime of natural protected areas, the conservation of natural habitats, wild fauna and flora. Among the rare species we mention: Coptosia albovittigera Heyden, Deroplia genei genei (Aragona), Phytoecia praetextata praetextata (Steven), Cerambyx miles Bonelli, C. welensii Kuster, Vadonia moesiaca Daniel \& Daniel, Cortodera differens Pic, Agapanthia kirbyi (Gyllenhal), Macroplea mutica (Fabricius), Clytra valeriana (Ménétries) and Cryptocephalus bohemius Drapiez.
\end{abstract}

Résumé. L'étude représente une synthèse des données sur la distribution des espèces de supra-famille Chrysomeloidea en Dobroudja (Dobrogea) région (Sud-Est de la Roumanie). 407 espèces de 132 genres et 15 sous-familles sont enregistrées, la base en étant des sources bibliographiques ainsi que l'étude du matériel conservé dans les collections de la «Grigore Antipa» Musée National d'Histoire Naturelle de Bucarest et l'Institut de Biologie Bucarest de l'Académie Roumaine. Les espèces Lilioceris lilii (Scopoli), Oulema erichsonii (Suffrian), Euluperus cyaneus (Joannis) et Altica quercetorum quercetorum Foudras sont mentionnés pour la première fois dans la faune de la Dobroudja. Certaines espèces endémiques sont mis en évidence: Vadonia hirsuta (K. Daniel \& J. Daniel), Dorcadion equestre transsilvanicum Ganglbauer, D. gashtarovi Sama, Dascălu \& Pesarini, D. axillare Küster and Brachyta balcanica Hampe. Rosalia alpina alpina Linnaeus, Morimus asper funereus Mulsant et Cerambyx cerdo cerdo Linnaeus sont des espèces protégées, inclus dans les annexes de la Directive du Conseil 92/43/CEE concernant la conservation des habitats naturels ainsi que de la faune et de la flore sauvage. Aussi, Brachyta balcanica Hampe, Pedostrangalia verticalis Germar et Neodorcadion exornatum (Frivaldsky von Frivald) sont des espèces d'intérêt national nécessitant une protection stricte, énumérés dans l'Ordonnance d'Urgence du Gouvernement no. 57/2007 concernant le régime des zones naturelles protégées, la conservation des habitats naturels, ainsi que de la faune et de la flore sauvage. Parmi les espèces rares nous mentionnons: Coptosia albovittigera Heyden, Deroplia genei genei (Aragona), Phytoecia praetextata praetextata (Steven), Cerambyx miles Bonelli, C. welensii Kuster, Vadonia moesiaca Daniel \& Daniel, Cortodera differens Pic, Agapanthia kirbyi (Gyllenhal), Macroplea mutica (Fabricius), Clytra valeriana (Ménétries) et Cryptocephalus bohemius Drapiez.

Key words: Coleoptera, Chrysomeloidea, Cerambycidae, Orsodacnidae, Chrysomelidae, Dobruja (Dobrogea), Romania. 


\section{INTRODUCTION}

Faunistic research is essential in assessing biodiversity in a given area and also for understanding processes at present occurring in nature related to the increasing anthropogenic impact.

Dobrogea (Dobruja) is a historical region shared by Bulgaria and Romania, located between the lower Danube river and the Black Sea, including the Danube Delta, Romanian coast and the northernmost part of the Bulgarian coast. The territory of Dobrogea comprises Northern Dobrogea, which is part of Romania, and Southern Dobrogea, which belongs to Bulgaria. The territory of the Romanian Dobrogea is organised as the counties of Constanţa and Tulcea, with a combined area of 15,500 $\mathrm{sq} \mathrm{km}$. The greater part of the Danube Delta lies in Romania (Tulcea county), while its northern part, on the left bank of the Chilia arm, is situated in Ukraine (Odessa Oblast). This includes the lagoons of Razim-Sinoe, which are located South of the proper delta. The continental climate is moderated by the Black Sea, and average temperatures range between $-4^{\circ} \mathrm{C}$ in January and $23^{\circ} \mathrm{C}$ in July.

Dobrogea presents a remarkable diversity of entomofauna shown by the large number of endemic species, recently described or recorded species and subspecies (Dascălu, 2010; Sama et al., 2010), rare species, and also by the high percentage of Pontic and Mediterranean elements (Skolka et al., 2005). Cerambycidae and Chrysomelidae families are, probably, among the most abundant in the Dobrogea region.

In this paper, we present a brief history of faunal investigations in Dobrogea, the most important phases of research, highlighting the contribution of each author to coleopterofauna research. The Coleoptera fauna was studied since the late $19^{\text {th }}$ century.

Among the foreigners who have settled in Romania, and had important contributions to the knowledge of Romanian entomofauna, we mention Maurice Jaquet (from Belgium) and Arnold Lucien Montandon (from France), eminent entomologist, Grigore Antipa's collaborator, at the Museum of Natural History in Bucharest.

The first data regarding the distribution of the chrysomelids and cerambycids from Dobrogea have been published by Montandon, Jaquet and Fleck.

In 1887 Montandon published the paper called "Excursions en Dobroudja" with exclusively reference to Dobrogea. Data regarding the distribution of the chrysomelids and cerambycids from the littoral of the Black Sea, Southern and Northern region, were published by the same author, in 1906 and 1908. Other works, including more data on the longhorn and leaf beetles from this region, are those published by Jaquet (1898 a, b; 1899; 1900 a, b; 1901; 1903) and Fleck (1905 a, b).

Eduard Fleck, born in Austria, studied natural sciences in Graz and Vienna. In 1894, he moved to Romania where he became director of a brewery at Azuga. $\mathrm{He}$ collected, in the Bucegi Mountains and Dobrogea $(1899,1903)$, and published the catalogue "Die Coleopteren Rumäniens" (1905). Fleck summarizes data published by Montandon, Jaquet and members of the Romanian Society of Naturalists, but also adds new data. His entomological collection, similar to that of Montandon, is preserved in the patrimony of "Grigore Antipa" National Museum of Natural History (Bucharest). Many subsequent authors used the data of these early scientific papers in their publications.

After almost 50 years, the previous data were completed with new ones, such as, the fascicle of Romanian Fauna (Panin \& Savulescu, 1961 - family 
Cerambycidae), as well as the papers signed by Ieniştea \& Negru (1956), Negru (1957), Săvulescu (1959). Also, we mentioned the syntheses on some coleopteran subfamilies and genera: subfamily Alticinae (Ionescu-Konnerth, 1963), genus Cryptocephalus (Roşca, 1973), genus Chrysomela (Panin, 1944; Roşca, 1974).

Between 1962 and 1965, the "Grigore Antipa" National Museum of Natural History initiated the study of entomofauna from Southern Dobrogea. The results were published by Săvulescu \& Popescu-Gorj (1964), Negru (1965), Negru \& Roşca (1967). These researches were resumed during 1993-1994 by Balog (1998) and by researchers from the Department of Entomology from the "Grigore Antipa" Museum, in Canaraua Fetii, Dumbrăveni, Negureni and Hagieni forests.

In the same period, the team from "Grigore Antipa" Museum has started entomofaunistic research in the Danube Delta Biosphere Reserve. Until 1968, very few data from the Danube Delta were published, due to adverse conditions in the first part of the $20^{\text {th }}$ century for field research. Data regarding the Coleoptera species from Letea sand bank were published by Negru and Ieniştea (1968), Săvulescu (1985), and those from Caraorman sand bank are found in Ieniştea's paper (1974). The studies on the longhorn and leaf beetles fauna of the Danube Delta have led to several papers (Serafim, 1993; Crişan 1993, 1994, 1995 a, b), and also contributed to the Catalogues of the collections from "Grigore Antipa" Museum (Serafim, 2005, 2006, 2007, 2009, 2010; Maican, 2006 a; Serafim \& Maican, 2011). Other contributions were given by Nagy (1972) whose data were taken over by Hoinic (1994) and Gomoiu \& Skolka (1998).

Information on the distribution of beetles from the Romanian littoral of the Black Sea are found in the papers published by Negru (1957), Nagy (1972), Gomoiu \& Skolka (1998) and Serafim \& Maican (2004).

Papers summarizing the beetles fauna in the entire region were published by Gruev (2001, 2002, 2005) and Skolka et al. (2005; 2006-2007).

\section{MATERIAL AND METHODS}

The paper presents data about the distribution of the species of Cerambycidae, Orsodacnidae and Chrysomelidae in Dobrogea region, based on bibliographical sources and on the study of material preserved in the collections of the "Grigore Antipa" National Museum of Natural History and the Institute of Biology Bucharest of the Romanian Academy.

Nomenclature and systematical order within the Cerambycidae family are in accordance with those used by Sama (2005) in Fauna Europaea, Hoskovec \& Rejzek (2009) in Longhorn beetles (Cerambycidae) of the West Palaearctic regio and Löbl \& Smetana (2010) in Catalogue of Palaearctic Coleoptera.

The arrangement of the taxa within the Orsodacnidae and Chrysomelidae families and the nomenclature follows the Catalogue of Palaearctic Coleoptera (op. cit.), namely: Silfverberg (2010) - family Orsodacnidae; Schmitt (2010) - subfamily Criocerinae; Borowiec \& Sekerka (2010) - subfamily Cassidinae; Kippenberg (2010) - subfamily Chrysomelinae; Beenen (2010) - subfamily Galerucinae; Döberl (2010) subfamily Alticinae; Regalin \& Medvedev (2010) - tribe Clytrini; Lopatin, Smetana, Schöller \& Löbl (2010) - tribe Cryptocephalini (including genus Cryptocephalus and remaining Cryptocephalini); Moseyko \& Sprecher-Uebersax (2010) - subfamily Eumolpinae. For each mentioned species, information about the collecting sites, geographical distribution and bibliographical sources are given. 
The general distribution of each species is given following Warchałowski (2003) and Löbl \& Smetana (op. cit.). For the species mentioned in old publications under another name, the taxonomy was updated. The authors of the previously published papers are presented in chronological order.

Collecting sites from the Danube Delta:

Buhaz sand bank; Caraorman; C.A. Rosetti; Chilia; Crişan; Doloşman Cape (Razim Lake); Dunavăț; Enisala; Fortuna Lake; Frasin sand bank; Şontea channel; Gorgova; Grindul Lupilor (sand bank, Sinoe Lake); Gura Portiței; Haşmacul lui Omer; Histria; Iancina Cape (Razim Lake); Jurilovca; Letea Forest (Haşmacul Mare, Haşmacurile Mici); Maliuc (Mila 26); Merheiul Mic Lake; Periprava; Popina Island (Razim Lake); Sacalin; Sărături sand bank; Sfântu Gheorghe; Slava Rusă; Sulina, Tulcea.

Abbreviations:

coll. - collection/s; E - East, Eastern; GANMNHB - "Grigore Antipa" National Museum of Natural History, Bucharest; NHMW - Natural History Museum, Wien; N - North, Northern; S - South, Southern; W - West, Western.

\section{RESULTS}

A total of 407 species from 132 genera and 15 subfamilies recorded from the Dobrogea region are listed.

The Chrysomelidae belong to 9 subfamilies as follows: Donaciinae - 12 species, Criocerinae - 12 species, Cassidinae - 17 species, Lamprosomatinae - one species, Chrysomelinae - 40 species, Galerucinae - 16 species, Alticinae - 96 species, Cryptocephalinae - 66 species, Eumolpinae - 6 species. 37 leaf beetles species, collected during the expeditions made by "Grigore Antipa" National Museum's team between 1993 and 1994 in the Dobrogea were identified by our late colleague, Cristina Hoinic.

The Cerambycidae belong to 6 subfamilies as follows: Prioninae - 2 species, Lepturinae - 32 species, Spondylidinae - 4 species, Necydalinae - 2 species, Cerambycinae - 44 species, Lamiinae - 55 species.

\section{Superfamily Chrysomeloidea Latreille, 1802}

Family Cerambycidae Latreille, 1802

Subfamily Prioninae Latreille, 1802

Tribe Aegosomatini J. Thomson, 1861

Aegosoma scabricorne Scopoli, 1763

Recorded: Măcin (Fleck, 1905 a); Letea Forest near Sulina (Montandon, 1906; Negru, 1968 a; Serafim, 2005), Turcoaia (Petri, 1912); Periprava (Negru, 1968 a; Serafim, 2005); Crişan (Ieniştea, 1974; Serafim, 2005), C.A. Rosetti (Negru, 1968 a; Serafim, 1993, 2005), Sfântu Gheorghe, Maliuc - Mila 26 (Serafim, 1993, 2005), Constanţa (Serafim \& Maican, 2004); Măcin, South of Dobrogea, Negureni Forest (Serafim, 2005); Măcin Mountains National Park (Măcin) (Skolka et al., 2006-2007). Distribution: Europe (except North); Asia (Near East).

Tribe Prionini Latreille, 1802

Prionus coriarius (Linnaeus, 1758)

Recorded: Greci (Montandon, 1908); Canaraua Fetii (Băneasa) (Panin \& Săvulescu, 1961; Serafim, 2005); South of Dobrogea, Negureni Forest (Serafim, 2005); Măcin Mountains National Park (Cetăţuia, Valea Fagilor Forest, Greci - Cautici) (Skolka et al., 2006-2007).

Distribution: Europe; North Africa; Asia (Near East, West Siberia). 
Subfamily Lepturinae Latreille, 1802

Tribe Lepturini Latreille, 1802

Anoplodera rufipes Schaller, 1783

Recorded: Esechioi, Fântâna Mare (Başpunar) (Serafim, 2006).

Distribution: Europe; Asia (the South Caucasus republics, East Siberia, Iran, Kazakhstan).

Anoplodera sexguttata Fabricius, 1775

Recorded: Esechioi, Fântâna Mare (Başpunar) (Serafim, 2006).

Distribution: Europe; North Africa (Algeria); Asia (Turkey, Georgia).

Grammoptera ruficornis ruficornis Fabricius, 1781

Recorded: Ciucurova Forest (Serafim, 2006).

Distribution: Europe; North Africa (Algeria, Tunisia); Asia (Turkey).

Judolia sexmaculata (Linnaeus, 1758)

Recorded: Babadag (Panin \& Săvulescu, 1961).

Distribution: Europe; Asia (Kazakhstan).

\section{Leptura quadrifasciata quadrifasciata Linnaeus, 1758}

Recorded: Dumbrăveni Forest, Valu lui Traian, Sfântu Gheorghe (Serafim, 2006).

Distribution: Europe; Asia.

Pachytodes erraticus erraticus Dalman, 1817

Recorded: Cocoş Monastery (Montandon, 1887; Fleck, 1905 b); Măcin, Ciucurova (Fleck, 1905 b; Panin \& Săvulescu, 1961); Babadag Forest (Fleck, 1905 b; Serafim, 2006); Oltina, Luncaviţa, Southern Dobrogea, Nifon, Hamcearca, Greci, Niculiţel, Valu lui Traian, Cocoș Monastery, Agighiol, Slava Rusă, Furnica, Dumbrăveni Forest (Serafim, 2006); Măcin Mountains National Park (Măcin) (Skolka et al., 2006-2007).

Distribution: Europe; Asia (Azerbaijan, Armenia, Georgia, Iran, Syria, Turkey).

Pedostrangalia (Neosphenalia) verticalis Germar, 1822

Recorded: Măcin (Fleck, 1905 b); Turcoaia (Igliţa) Lake (Montandon, 1906); Babadag Forest (Fleck, 1905 b; Panin \& Săvulescu, 1961; Serafim, 2006; Serafim \& Maican, 2011); Celic Dere (Serafim, 2006), Măcin Mountains National Park (Măcin, Greci, Pricopan) (Skolka et al., 2006-2007).

Distribution: Europe (Balkan Peninsula, Italy, Slovenia); Asian Turkey. Pseudovadonia livida livida Fabricius, 1776

Recorded: Cocoş Monastery (Montandon, 1887; Fleck, 1905 b; Serafim, 2006); Mangalia (Jaquet, 1900 a); Ciucurova (Fleck, 1905 b); Comarova Forest (Mangalia) (Negru \& Roşca, 1967; Serafim \& Maican, 2004; Serafim, 2006); Caraorman Forest (Ieniştea, 1974; Serafim, 2006); C.A. Rosetti, Sulina (Serafim, 1993, 2006); Agigea (Serafim \& Maican, 2004); Valu lui Traian, Eforie Sud (Carmen Sylva), Nifon, Hamcearca, Oltina, Esechioi, Hagieni Forest, Techirghiol, Constanța (Palas), Greci, Canaraua Fetii (Băneasa), Periprava, Măcin, Niculițel, Sulina, Furnica, Dumbrăveni Forest, Negureni Forest (Serafim, 2006); Măcin Mountains National Park (Măcin, Nifon) (Skolka et al., 2006-2007).

Distribution: Europe; Asia.

Rutpela maculata maculata Poda von Neuhaus, 1761

Recorded: Cocoş Monastery (Montandon, 1906); Măcin Mountains National Park (Măcin, Greci, Pricopan) (Skolka et al., 2006-2007).

Distribution: Europe; Asia (Turkey, Azerbaijan, Armenia, Georgia, Iran, Kazakstan). Stenurella bifasciata bifasciata O.F. Müller, 1776

Recorded: Greci, Cerna (Montandon, 1887); Pricopan crest near Măcin (Jaquet, 1900 a; Fleck, 1905 b); Cocoş Monastery (Montandon, 1887, 1906); Comarova Forest 
(Mangalia), Agigea (Serafim \& Maican, 2004; Serafim, 2006); Măcin, Oltina, Valu lui Traian, Nifon, Sfântu Gheorghe, Southern Dobrogea, Hagieni Forest, Canaraua Fetii (Băneasa), Niculiţel, Agighiol, Beştepe, Slava Rusă (Serafim, 2006); Babadag Forest (Serafim, 2006; Serafim \& Maican, 2011); Măcin Mountains National Park (Greci, Nifon) (Skolka et al., 2006-2007).

Distribution: Europe, Asia (East Siberia, Iran, Iraq, Kazakhstan).

Stenurella melanura Linnaeus, 1758

Recorded: Cocoş Monastery (Montandon, 1887); Olimp resort (North Mangalia) (Serafim \& Maican, 2004); Canaraua Fetii (Băneasa), Valu lui Traian, Oltina, Luncavița, Greci, Southern Dobrogea, Nifon, Hamcearca, Hagieni Forest, Niculițel, Dumbrăveni Forest (Serafim, 2006); Babadag Forest (Serafim, 2006; Serafim \& Maican, 2011); Măcin Mountains National Park (Greci) (Skolka et al., 2006-2007). Distribution: Europe; Asia (Turkey, the South Caucasus republics, East and West Siberia, Russia - Far East, Iran, Kazakhstan, Mongolia, China).

Stenurella nigra Linnaeus, 1758

Recorded: Babadag (Fleck, 1905 b); Canaraua Fetii (Băneasa) (Balog, 1998; Serafim, 2006); Măcin, Esechioi, Cocoş Monastery, Ciucurova Forest, Celic Dere (Serafim, 2006).

Distribution: Europe; Asia (the South Caucasus republics, Iran, Turkey).

Stenurella septempunctata septempunctata (Fabricius, 1792)

Recorded: Cocoş Monastery (Fleck, 1905 b; Serafim, 2006); Agigea (Serafim \& Maican, 2004); Luncavița, Oltina, Babadag Forest, Greci, Southern Dobrogea, Hagieni Forest, Canaraua Fetii (Băneasa), Sfântu Gheorghe, Niculiţel, Slava Rusă, Dumbrăveni Forest, Negureni Forest (Serafim, 2006).

Distribution: Europe (except North); Near East.

Stictoleptura cordigera cordigera (Fuessly, 1775)

Recorded: Măcin (Fleck, 1905 b; Montandon, 1906); Băneasa (Togănel, 2004); Iortmac Lake, Southern Dobrogea, Oltina, Canaraua Fetii (Băneasa), Niculițel, Cocoş Monastery (Serafim, 2006); Măcin Mountains National Park (Măcin) (Skolka et al., 2006-2007).

Distribution: Europe (Central, South and South-East); North Africa (Libya); Asia (Turkey, the Caucasus republics, Iran, Syria, Lebanon, Iraq, Israel, Libya, Cyprus).

Stictoleptura fulva (De Geer, 1775)

Recorded: Southern Dobrogea (Serafim, 2006).

Distribution: Europe; Asia (Turkey).

Stictoleptura rubra rubra (Linnaeus, 1758)

Recorded: Babadag, Letea Forest, Periprava (Serafim, 2006).

Distribution: Europe; Asia (East and West Siberia, Kazakhstan, Korea).

Stictoleptura scutellata scutellata (Fabricius, 1781)

Recorded: Babadag Forest (Serafim, 2006).

Distribution: Europe; Asia (Turkey).

Strangalia attenuata (Linnaeus, 1758)

Recorded: Cocoş Monastery (Montandon, 1887; Serafim, 2006); Periprava (Negru, 1968 a; Serafim, 1993, 2006); C.A. Rosetti, Sulina (Serafim, 1993, 2006); Hagieni Forest (Serafim \& Apetrei, 1996; Serafim et al., 2004); Danube Delta, Canaraua Fetii (Băneasa), Southern Dobrogea, Babadag Forest, Greci, Caraorman, Niculiţel, Sfântu Gheorghe (Serafim, 2006).

Distribution: Europe, Asia. 
Vadonia bipunctata globicollis (Desbrochers des Loges, 1870)

Recorded: Măcin, Turcoaia (Igliţa) Lake (Montandon, 1906); Eforie (Carmen Sylva) (Knechtel \& Panin, 1944); Târguşor (Ester) (Montandon, 1906; Panin \& Săvulescu, 1961; Serafim \& Maican, 2004); Mangalia, Agigea (Panin \& Săvulescu, 1961; Serafim \& Maican, 2004); Tulcea, Ilgani (Panin \& Săvulescu, 1961); Periprava (Ieniştea, 1968; Negru, 1968 a; Serafim, 1993, 2006); C.A. Rosetti (Serafim, 1993, 2006; Togănel, 2004); Letea Forest (Haşmacul Mare) (Ieniştea, 1968; Serafim, 1993, 2006); Caraorman, Sulina (Serafim, 1993); Hagieni Forest (Serafim \& Apetrei, 1996); Mangalia, Valu lui Traian, Murfatlar, Agigea, Canaraua Fetii (Băneasa), Comarova Forest (Mangalia), Sălcioara (6 Martie) Lake (Ianola Cape) (Serafim, 2006); Măcin Mountains National Park (Măcin) (Skolka et al., 2006-2007).

Distribution: Europe (Republic of Moldova, Romania, Russia - South European Territory, Ukraine).

Remarks. In the papers published before 2007, the species is cited as Vadonia steveni. Vadonia hirsuta (K. Daniel \& J. Daniel, 1891)

Recorded: Hârşova (Montandon, 1906); Dobrogea (Panin \& Săvulescu, 1961; Balaci, 2000); Murfatlar (Serafim, 2006); Măcin Mountains National Park (Măcin, Greci, Pricopan) (Skolka et al., 2006-2007).

Distribution: Europe (Romania).

Vadonia moesiaca (K. Daniel \& J. Daniel, 1891)

Recorded: Hagieni, Babadag (Dascălu, 2010).

Distribution: Albania, Bulgaria, Greece, Macedonia, Serbia, European and Asian Turkey.

\section{Vadonia unipunctata unipunctata (Fabricius, 1787)}

Recorded: Constanța (Jaquet, 1900 a; Fleck, 1905 b; Knechtel \& Panin, 1944); Mangalia, Techirghiol (Jaquet, 1900 a; Fleck, 1905 b; Knechtel \& Panin, 1944; Panin \& Săvulescu, 1961); Orliga (Jaquet, 1900 a; Fleck, 1905 b); Turcoaia (Igliţa) Lake (Montandon, 1906); Târguşor (Ester) (Montandon, 1906; Serafim, 2006); Măcin, Valu lui Traian, Murfatlar, Hagieni Forest, Caraorman Forest (Serafim, 2006); Măcin Mountains National Park (Măcin, Greci, Pricopan) (Skolka et al., 2006-2007).

Distribution: Europe (Austria, Balkan Peninsula, Russia, Czech Republic, France, Hungary, Italy, Republic of Moldova, Poland, Turkey, Slovakia, Spain, Ukraine); Asia (Armenia, Georgia, Kazakhstan, Turkey).

Tribe Oxymirini Danilevsky, 1997

Oxymirus cursor (Linnaeus, 1758)

Recorded: Sulina (Serafim, 2005).

Distribution: Europe; Asia (West Siberia, China).

\section{Tribe Rhagiini Kirby, 1837}

Anisorus quercus quercus (Götz, 1783)

Recorded: Greci, South of Dobrogea, Cocoş Monastery (Serafim, 2005); Babadag Forest (Serafim \& Maican, 2011).

Distribution: Europe; Asia (Turkey, Georgia, East and West Siberia, Mongolia).

Brachyta balcanica Hampe, 1870

Recorded: Murfatlar (Fleck, 1905 a; Serafim, 2005); Agigea (Panin \& Săvulescu, 1961); Dobrogea (Balaci, 2000; Serafim \& Maican, 2004; Serafim, 2005); Hagieni Forest (Serafim, 2005).

Distribution: Europe (Albania, Bulgaria, Greece, Romania, Turkey, Serbia and Montenegro); Asia (Turkey).

Cortodera differens Pic, 1898

Recorded: Forest border to the S from Babadag village (Dascălu, 2010). 
Distribution: Greece, Turkey, Romania.

Cortodera humeralis Schaller, 1783

Recorded: Popina Island (Razim Lake) (Serafim, 1993).

Distribution: Central and South-eastern Europe, Turkey.

Dinoptera collaris (Linnaeus, 1758)

Recorded: Pricopan, Ciucurova (Fleck, 1905 a; Panin \& Săvulescu, 1961); Măcin (Montandon, 1906); Comarova Forest (Mangalia) (Negru \& Roşca, 1967; Serafim \& Maican, 2004; Serafim, 2005); Sălcioara (6 Martie) Forest (Jurilovca) (Serafim, 1993); Canaraua Fetii (Băneasa) (Balog, 1998; Serafim, 2005); Agigea (Serafim \& Maican, 2004); Cernavodă, Măcin, Greci, Hagieni Forest (Serafim, 2005); Dumbrăveni Forest, Niculițel, Babadag Forest, Cocoş Monastery, Negureni Forest, Dumbrăveni Forest, Celic Dere Monastery, Valea Fagilor Forest near Luncaviţa (Serafim, 2005); Măcin Mountains National Park (Skolka et al., 2006-2007).

Distribution: Europe; Asia (Azerbaijan, Armenia, Georgia, East and West Siberia, Iran, Kazakhstan, Turkey, China). Rhagium (Megarhagium) mordax (De Geer, 1775)

Recorded: Cernavodă (Serafim, 2005).

Distribution: Europe; Asia (Russia - East and West Siberia, Kazakhstan).

Rhagium (Rhagium) inquisitor inquisitor (Linnaeus, 1758)

Recorded: Techirghiol (Jaquet, 1900 a; Fleck, 1905 a).

Distribution: Europe; Asia (Russia - East and West Siberia, Kazakhstan, Mongolia); Nearctic Region.

Stenocorus meridianus (Linnaeus, 1758)

Recorded: Babadag (Jaquet, 1901; Fleck, 1905 a; Greci (Montandon, 1908); Agigea (Serafim \& Maican, 2004; Serafim, 2005); South of Dobrogea (Serafim, 2005); Măcin Mountains National Park (Măcin, Greci, Pricopan) (Skolka et al., 2006-2007) Distribution: Europe; Asia (East and West Siberia, Kazakhstan, Korea).

Tribe Rhamnusiini Sama, 2009

Rhamnusium bicolor bicolor (Schrank, 1781)

Recorded: Dobrogea (without other data) (Fleck, 1905 a).

Distribution: Europe; Kazakhstan.

Subfamily Spondylidinae Audinet-Serville, 1832

Tribe Anisarthrini Mamaev \& Danilevsky, 1973

Anisarthron barbipes (Schrank, 1781)

Recorded: Constanța (Panin \& Săvulescu, 1961); Tuzla (Serafim \& Maican, 2004; Serafim, 2007); Dobrogea (without other data) (Skolka et al., 2005).

Distribution: Europe (Balkan Peninsula, Czech Republic, France, Germany, Hungary, Italy, Poland, Slovakia, Switzerland, Ukraine).

Tribe Asemini J. Thomson, 1861

Arhopalus rusticus (Linnaeus, 1758)

Recorded: Hagieni Forest, Dumbrăveni Forest, Furnica (Serafim, 2007).

Distribution: Europe; Asia; North Africa; Nearctic; Oriental Regions. Asemum striatum (Linnaeus, 1758)

Recorded: Dumbrăveni Forest, Furnica (Serafim, 2007).

Distribution: Europe; Asia (Near East, Central Asia, East Palaearctic); Nearctic Region; Neotropical Region.

Tribe Spondylidini Audinet-Serville, 1832

Spondylis buprestoides (Linnaeus, 1758)

Recorded: Canaraua Fetii (Băneasa) (Balog, 1998). 
Distribution: Europe; Asia; North Africa; Oriental Region.

Subfamily Necydalinae Latreille, 1825

Necydalis major major Linnaeus, 1758

Recorded: Mangalia (Serafim \& Maican, 2004).

Distribution: Europe; Asia (East and West Siberia, Russia Far East, Kazakhstan, Mongolia, Korea, China).

Necydalis ulmi Chevrolat, 1838

Recorded: Mangalia (Panin \& Săvulescu, 1961; Serafim \& Maican, 2004).

Distribution: Europe; Asian Turkey.

Subfamily Cerambycinae Latreille, 1802

Tribe Callichromatini Swainson \& Shuckard, 1840

Aromia moschata moschata Linnaeus, 1758

Recorded: Sfântu Gheorghe (Negru, 1968 a; Serafim, 1993, 2009); Periprava (Serafim, 1993, 2009); Caraorman (Serafim, 1993, 2009); Dobrogea (without other data) (Skolka et al., 2005); Southern Dobrogea, Oltina, Letea Forest (Serafim, 2009). Distribution: Europe, Near East.

Tribe Callidiini Kirby, 1837

Callidium aeneum aeneum (De Geer, 1775)

Recorded: Hagieni Forest (Serafim, 2009).

Distribution: Europe; Asia (Near East, East and West Siberia).

Callidium violaceum (Linaeus, 1758)

Recorded: Sfântu Gheorghe (Serafim, 2009).

Distribution: Europe; Asia.

Leioderes kollari Redtenbacher, 1849

Recorded: Eforie Sud (Panin \& Săvulescu, 1961).

Distribution: West, Central and South-East Europe, North Europe (only Norwey, Sweden); Near East (Turkey).

Phymatodes testaceus (Linnaeus, 1758)

Recorded: Turcoaia (Igliţa) Lake (Fleck, 1905 b); Caraorman (Ieniştea, 1974);

Sulina (Serafim, 1993, 2009); Valu lui Traian, Eforie Sud, Esechioi, Hagieni Forest, Ciucurova (Serafim, 2009); Măcin Mountains National Park (Măcin, Greci, Pricopan) (Skolka et al., 2006-2007).

Distribution: Europe; Asia (Near East, Russia Far East, East and West Siberia, Kazakhstan); North Africa, Nearctic Region, Oriental Region.

Poecilium alni alni (Linnaeus, 1767)

Recorded: Măcin (Montandon, 1906); Periprava, Letea Forest (Negru, 1968 a, b);

Măcin Mountains National Park (Skolka et al., 2006-2007)

Distribution: Europe; Asia (Turkey, Kazakhstan).

Poecilium fasciatum (Villers, 1789)

Recorded: Periprava, Letea Forest (Haşmacurile Mici) (Negru, 1968 a).

Distribution: Europe (Austria, Balkan Peninsula, Czech Republic, France, Germany, Hungary, Italy, Slovakia, Spain, Switzerland, Ukraine); Asia (Near East).

Poecilium pusillum pusillum (Fabricius, 1787)

Recorded: Periprava, Letea Forest (Haşmacurile Mici) (Negru, 1968 a).

Distribution: Europe (except North).

Pyrrhidium sanguineum (Linnaeus, 1758)

Recorded: Murfatlar (Hurmuzachi, 1904); Ostrov (Panin \& Săvulescu, 1961); Sfântu Gheorghe (Serafim, 2009). 
Distribution: Europe; Asia (Near East); North Africa.

Ropalopus clavipes (Fabricius, 1775)

Recorded: Pricopan Crest near Măcin (Jaquet, 1900 a; Fleck, 1905 b; Panin \& Săvulescu, 1961; Skolka et al., 2006-2007); Canaraua Fetii (Băneasa) (Panin \& Săvulescu, 1961); Periprava (Panin \& Săvulescu, 1961; Negru, 1968 a); Sulina (Ieniştea, 1974); Caraorman Forest (Ieniştea, 1974; Serafim, 2009); Hagieni Forest, Iortmac Lake, Esechioi, Sfântu Gheorghe, Negureni Forest (Serafim, 2009); Măcin Mountains National Park (Măcin, Greci) (Skolka et al., 2006-2007).

Distribution: Europe (except Great Britain, Croatia, Iceland, Scandinavian countries), Asia (Near East, Kazakhstan).

Ropalopus insubricus insubricus (Germar, 1824)

Recorded: Southern Dobrogea (Serafim, 2009).

Distribution: Europe (Austria, Balkan Peninsula, Czech Republic, France, Hungary, Italy, Slovenia, Spain, Serbia and Montenegro).

Ropalopus macropus (Germar, 1824)

Recorded: C.A. Rosetti (Serafim, 1993, 2009); Esechioi, Canaraua Fetii (Băneasa), Cocoş Monastery (Serafim, 2009).

Distribution: Central and South Eastern Europe; Asia (Near East).

Tribe Cerambycini Latreille, 1802

Cerambyx cerdo cerdo Linnaeus, 1758

Recorded: Turcoaia (Igliţa) Lake (Fleck, 1905 b); Comarova Forest (Mangalia) (Negru \& Roșca, 1967); Dobrogea (without other data) (Skolka et al., 2005); Canaraua Fetii (Băneasa) (Serafim, 2009; Tatole et al., 2009); Măcin Mountains National Park (Cetăţuia, Valea Fagilor Forest, Ghiunaltu Peak) (Skolka et al., 2006-2007); Măcin Mountains, North Dobrogea Plateau (Tatole et al., 2009); Măcin Mountains, Greci (Tăuşan \& Bucşa, 2010).

Distribution: Europe (except Denmark, Estonia, Iceland, Lithuania, Norway); Asia (the Caucasian Republics, Near East); North Africa (Morocco).

Cerambyx miles Bonelli, 1812

Recorded: Canaraua Fetii (Băneasa) (Săvulescu, 1969; Serafim, 2009); Cocoş Monastery, Caugagia (Serafim, 2009).

Distribution: Europe (Austria, Balkan Peninsula, Turkey, France, Hungary, Italy, Slovakia, Slovenia, Spain, Switzerland, Ukraine), Asia (Azerbaijan, Armenia, Georgia, Turkey).

Cerambyx scopolii Fusslins, 1775

Recorded: Babadag (Fleck, 1905 b); Dobrogea (without other data) (Skolka et al., 2005); Valea Fagilor Forest near Luncavița (Serafim, 2009); Măcin Mountains National Park (Skolka et al., 2006-2007).

Distribution: Europe (except Finland, Iceland, Ireland); Asia (The Caucasian Republics, Syria, Turkey).

\section{Cerambyx welensii Kuster, 1846}

Recorded: Canaraua Fetii (Băneasa) (Săvulescu, 1969; Serafim, 2009).

Distribution: Europe (Balkan Peninsula, France, Hungary, Italy, Malta, Portugal, Sicily, Slovakia, Slovenia, Spain, Ukraine); Asia (Near East).

Tribe Clytini Mulsant, 1839

Chlorophorus figuratus (Scopoli, 1763)

Recorded: Cocoş Monastery (Montandon, 1987, 1906; Serafim, 2009); Babadag Forest (Fleck, 1905 b; Panin \& Săvulescu, 1961; Serafim, 2009); Greci (Montandon, 1908); Comarova Forest (Mangalia) (Serafim \& Maican, 2004); Canaraua Fetii (Băneasa) (Balog, 1998; Serafim, 2009); Dobrogea (without other data) (Skolka 
et al., 2005); Măcin Mountains National Park (Nifon) (Skolka et al., 2006-2007); Southern Dobrogea, Esechioi, Beştepe, Dumbrăveni Forest (Serafim, 2009).

Distribution: Europe (except North, Portugal, Mediterranean islands), Asia (Iran, East Siberia, Kazakhstan, China).

Chlorophorus herbstii (Brahm, 1790)

Recorded: Măcin (Montandon, 1906).

Distribution: Europe; Asia (Turkey, Kazakhstan, East and West Siberia).

Chlorophorus sartor (Müller, 1766)

Recorded: Cocoş Monastery (Montandon, 1887, 1906); Comarova Forest (Mangalia) (Panin \& Săvulescu, 1961; Negru \& Roşca, 1967; Serafim \& Maican, 2004; Serafim, 1993, 2009); Periprava (Negru, 1968 a; Serafim, 2009); Constanţa, Agigea, Olimp resort (North Mangalia) (Serafim \& Maican, 2004; Serafim, 2009); Dobrogea (without other data) (Skolka et al., 2005); Valu lui Traian, Canaraua Fetii (Băneasa), Oltina, Techirghiol, Greci, Cogeacu, Tulcea, Caraorman, Cocoş Monastery, Esechioi Forest, Somova, Isaccea, Hagieni Forest, Dumbrăveni (Serafim, 2009).

Distribution: Europe (except North, East and Northwest Russia), Asia (East and West Siberia, Kazakhstan, Turkmenistan, China; Near East).

\section{Chlorophorus varius varius (Müller, 1766)}

Recorded: Măcin (Montandon, 1887, 1908; Serafim, 2009); Cocoş Monastery (Montandon, 1887, 1908); Comarova Forest (Mangalia) (Negru, 1957; Negru \& Roşca, 1967; Serafim, 2009); Chilia Veche (Negru, 1968 a); C.A. Rosetti (Negru, 1968 a); Caraorman (Ieniştea, 1974; Serafim, 2009); Periprava (Serafim, 1993, 2009); Tulcea (Serafim, 1993); Sfântu Gheorghe (Serafim, 1993); Agigea, Olimp resort (North Mangalia) (Serafim \& Maican, 2004; Serafim, 2009); Fântâniţa Nature Reserve (Vasiliu-Oromulu et al., 2004); Dobrogea (without other data) (Skolka et al., 2005); Măcin Mountains National Park (Nifon) (Skolka et al., 2006-2007); Valu lui Traian, Canaraua Fetii (Băneasa), Oltina, Techirghiol, Southern Dobrogea, Murfatlar, Palas Constanţa, Tulcea, Letea Forest, Chilia, Sulina, C.A. Rosetti, Hagieni Forest, Niculiţel, Crişan, Sfântu Gheorghe, Cocoş Monastery, Somova, Saon marsh, Dunărea Veche, Gorgova sand bank, Beștepe, Canaraua Fetii (Băneasa), Negureni Forest, Sărături sand bank, Dumbrăveni Forest, Babadag Forest (Serafim, 2009).

Distribution: Europe (except North, Portugal, Northwest Russia); Asia (The Caucasian Republics, Russia Far West, West Siberia, Kazakhstan, China, Turkey).

\section{Clytus arietis arietis (Linnaeus, 1758)}

Recorded: C.A. Rosetti - Letea Forest (Serafim, 1993, 2009); Hagieni Forest (Serafim \& Apetrei, 1996); Agigea (Serafim \& Maican, 2004); Dobrogea (without other data) (Skolka et al., 2005); Cocoş Monastery (Serafim, 2009).

Distribution: Europe; North Africa (Madeira Archipelago).

Clytus rhamni Germar, 1817

Recorded: Dobrogea (without other data) (Montandon, 1908; Skolka et al., 2005); Techirghiol (Panin \& Săvulescu, 1961); C.A. Rosetti (Serafim, 1993, 2009); Comarova Forest (Mangalia), Agigea (Serafim \& Maican, 2004; Serafim, 2009); Măcin Mountains National Park (Greci) (Skolka et al., 2006-2007); Babadag Forest, Canaraua Fetii (Băneasa), Oltina, Valu lui Traian, Murfatlar, Hagieni Forest, Dumbrăveni (Serafim, 2009).

Distribution: Europe (except Great Britain, Scandinavian Republics, Estonia, Iceland); Asia (the Caucasian Republics, Near East, Kazakhstan). 
Isotomus speciosus (Schneider, 1787)

Recorded: Cocoş Monastery (Montandon, 1987, 1906); Greci, (Montandon, 1908); Iortmac Lake (Săvulescu, Popescu-Gorj, 1964); Tulcea (Serafim, 1993), Fântâna Mare (Başpunar), Babadag Forest (Serafim, 2009).

Distribution: Europe (except North and West); Asia (The Caucasian Republics, Turkey)

Plagionotus arcuatus (Linnaeus, 1758)

Recorded: Esechioi, Iortmac Lake, Ciucurova (Serafim, 2009); Visterna, Babadag Forest (Serafim \& Maican, 2011).

Distribution: Europe; Asia (Near East, Kyrgyzstan, Kazakhstan); North Africa.

Plagionotus bobelayei (Brullé, 1832)

Recorded: Danube Delta: Frasin sand bank, Buhaz sand bank (Panin \& Săvulescu, 1961; Săvulescu, 1985); Chilia Veche, Letea Forest (Negru, 1968 a); Periprava (Negru, 1968 a; Serafim, 1993, 2009); Sarinasuf (Serafim, 1993); C.A. Rosetti (Negru, 1968 a; Serafim, 1993, 2009); Caraorman (Serafim, 1993, 2009); Sfântu Gheorghe, Sfiştofca, Dunavăț (Serafim, 2009).

Distribution: Europe (The Balkan Peninsula, Russia - South European Territory, Ukraine); Asia (Near East, Turkmenistan).

Plagionotus detritus (Linnaeus, 1758)

Recorded: Eforie Sud (Panin \& Săvulescu, 1961); Caraorman (Serafim, 1993); Sfântu Gheorghe (Serafim, 2009).

Distribution: Europe; Asia (the Caucasian Republics, Turkey, Syria, Kazakhstan). Plagionotus floralis (Pallas, 1773)

Recorded: Mangalia, Techirghiol (Jaquet 1899, 1901; Fleck, 1905 b; Negru \& Roşca, 1967; Serafim, 2009); Babadag Forest (Fleck, 1905 b); Măcin (Fleck, 1905 b; Serafim, 2009); Turcoaia (Igliţa) Lake (Montandon, 1906); Comarova Forest (Mangalia) (Negru \& Roşca, 1967); Merheiul Mic Lake, Enisala (Serafim, 1993); Periprava, C.A. Rosetti, Letea, Enisala (Serafim, 1993, 2009); Hagieni Forest (Serafim \& Apetrei, 1996; Serafim, 2009); Constanţa, Agigea, Olimp resort (North Mangalia) (Serafim \& Maican, 2004); Valu lui Traian, Canaraua Fetii (Băneasa), Murfatlar, Periprava, Fântâna Mare (Başpunar), Negureni Forest, Greci, Niculițel, Agighiol, Gura Dobrogei, Capul Iancila, Sălcioara (6 Martie) Lake, Beştepe, Dumbrăveni Forest (Serafim, 2009); Măcin Mountains National Park (Greci, Nifon) (Skolka et al., 2006-2007).

Distribution: Europe (Austria, Balkan Peninsula, Czech Republic, France, Hungary, Italy, Latvia, Lithuania, Republic of Moldova, Poland, Russia, Slovakia, Ukraine); Asia (The Caucasian Republics, East Siberia, Near East, Central and East Asia).

Rusticoclytus pantherinus (Savenius, 1825)

Recorded: Danube Delta, Sfântul Gheorghe arm - Buhaz sand bank (Panin \& Săvulescu, 1961; Serafim, 1993, 2009).

Distribution: Europe (Austria, Belarus, Czech Republic, Finland, France, Germany, Hungary, Italy, Norway, Poland, Russia, Slovakia, Sweden, Switzerland); Asia (East Siberia, Russia Far East, Kazakhstan, Mongolia, China).

Rusticoclytus rusticus (Linnaeus, 1758)

Recorded: Danube Delta (Săvulescu, 1985); the Danube Delta, Sfântul Gheorghe arm - Buhaz sand bank, Sfântu Gheorghe (Serafim, 1993).

Distribution: Europe, Asia, North Africa. 
Xylotrechus antilope antilope (Schönherr, 1817)

Recorded: Babadag (Fleck, 1905 b; Panin \& Săvulescu, 1961); Maliuc - Mila 26 (Serafim, 1993); Canaraua Fetii (Băneasa), Southern Dobrogea, Dobromir, Hagieni Forest (Serafim, 2009).

Distribution: Europe (except Denmark, Estonia, Finland, Iceland, Ireland, Macedonia, East, North and Northwest Russia); Asia (Azerbaijan, Armenia, Georgia, Iran, Turkey); North Africa.

Xylotrechus arvicola (Olivier, 1795)

Recorded: Greci (Montandon, 1908); Fântâna Mare (Başpunar), Iortmac Lake, Canaraua Fetii (Băneasa) (Panin \& Săvulescu, 1961); Esechioi (Serafim, 2009); Măcin Mountains National Park (Măcin, Greci, Pricopan) (Skolka et al., 2006-2007). Distribution: Europe (except Scandinavian countries, Iceland, Ireland); Asia (Azerbaijan, Armenia, Georgia, Syria, Turkey, Kazakhstan); North Africa (Alger, Morocco).

Tribe Graciliini Mulsant, 1839

Axinopalpis gracilis gracilis (Krynicki, 1832)

Recorded: Eforie Sud (Panin \& Săvulescu, 1961); Babadag Forest (Serafim, 2009).

Distribution: Europe; Asia (Near East).

Tribe Hesperophanini Mulsant, 1839

Trichoferus campestris Faldermann, 1835

Material: 1 spec., Agigea Nature Reserve, 16.VII.2003, leg. Rodica Serafim (GANMNHB coll.) (Dascălu \& Serafim, unpublished data).

Distribution: The natural range of Trichoferus campestris extends from Japan, China, Korea and Central Asia to the Caucasus. This Asian invasive species was detected in the last twenty years in several European countries.

Remarks. Trichoferus campestris was published firstly as Trichoferus griseus by Serafim \& Maican (2004). The first correct record of T. campestris in Romania appears in the Catalogue of Palaearctic Coleoptera (Sama \& Löbl, 2010).

Trichoferus fasciculatus (Faldermann, 1837)

Recorded: Constanţa (Ieniştea \& Negru, 1956; Panin \& Săvulescu, 1961).

Distribution: Europe (West, East, Central); Asia (Near East), North Africa.

Remarks. In the mentioned papers the species was cited as the Trichoferus griseus var. fasciculatus (Faldermann).

Tribe Hylotrupini Zagajkevich, 1991

Hylotrupes bajulus (Linnaeus, 1758)

Recorded: Tulcea (Montandon, 1887, 1906); Comarova Forest (Mangalia) (Negru \& Roșca, 1967); Sulina (Negru, 1968 a); Caraorman (Ieniștea, 1974; Serafim, 2009); Periprava, Sfântu Gheorghe (Serafim, 1993, 2009); Constanţa (Serafim \& Maican, 2004); Bugeac Lake, Canaraua Fetii (Băneasa), Constanţa, Dumbrăveni Forest (Serafim, 2009); Măcin Mountains National Park (Măcin, Greci, Pricopan) (Skolka et al., 2006-2007).

Distribution: Palaearctic, Afrotropical, Nearctic, Neotropical, Oriental, Australian Regions.

Tribe Molorchini Gistel, 1848

Glaphyra kiesenwetteri kiesenwetteri (Mulsant \& Rey, 1861)

Recorded: Comarova Forest (Mangalia) (Negru, 1965; Negru \& Roşca, 1967; Serafim \& Maican, 2004); Canaraua Fetii (Băneasa) (Serafim, 2009).

Distribution: Europe (Austria, the Balkan Peninsula, Czech Republic, France, Germany, Hungary, Italy, Slovakia, Russia - South European Territory, Switzerland, Ukraine). 
Glaphyra umbellatarum (Schreber, 1759)

Recorded: Hagieni Forest, Ciucurova Forest (Serafim, 2009).

Distribution: Europe (except Albania, Portugal, Norway, Ireland. Iceland); Asia (the Caucasian Republics, Turkey, Turkmenistan).

Tribe Nathriini Arnett, 1962

Obrium cantharinum cantharinum (Linnaeus, 1767)

Recorded: Eforie Sud (Panin \& Săvulescu, 1961); Periprava (Negru, 1968 a).

Distribution: Europe; the Caucasus; Asia (Russia - East Siberia and far East, Kazakhstan, Mongolia, China).

Tribe Purpuricerini J. Thomson, 1861

Calchaenesthes oblongomaculata (Guérin-Méneville, 1844)

Recorded: Esechioi (Panin \& Săvulescu, 1961; Serafim, 2009).

Distribution: Europe (Bulgaria, Greece); Near East (Cyprus, Jordan, Turkey).

Purpuricenus kaehleri kaehleri (Linnaeus, 1758)

Recorded: Măcin (Fleck, 1905 b; Skolka et al., 2006-2007); Fântâna Mare (Başpunar), Canaraua Fetii (Băneasa), Southern Dobrogea, Negureni Forest (Serafim, 2009); Măcin Mountains National Park (Cetăţuia) (Skolka et al., 2006-2007).

Distribution: Europe; Asia (the Caucasian Republics, Turkey).

Tribe Rosaliini Fairmaire, 1864

Rosalia alpina alpina (Linnaeus, 1758)

Recorded: Pricopan (Fleck, 1905 b; Skolka et al., 2006-2007); Greci (Montandon, 1908); Sulina (Ieniştea, 1974); Măcin Mountains National Park (Măcin, Ghiunaltu Peak, Chediu plateau) (Skolka et al., 2006-2007).

Distribution: Europe (except North); Asia (The Caucasian Republics, Turkey).

Tribe Stenopterini Gistel, 1848

Lampropterus femoratus (Germar, 1824)

Recorded: Greci (Montandon, 1908); Fântâna Mare (Başpunar), Canaraua Fetii (Băneasa), Hagieni Forest (Serafim, 2009).

Distribution: Europe (Albania, Balkan Peninsula, Russia - South European Territory, Ukraine); Asia (Cyprus, Azerbaijan, Armenia, Georgia, Iran, Israel, Lebanon, Syria, Turkey).

Stenopterus ater (Linnaeus, 1767)

Recorded: Pricopan crest near Măcin (Jaquet, 1900 a; Fleck, 1905 b; Skolka et al., 2006-2007); Măcin Mountains National Park (Măcin, Greci) (Skolka et al., 20062007).

Distribution: Europe (Balkan Peninsula, France, Italy, Portugal, Sardinia, Sicily, Slovakia, Spain, Switzerland, Ukraine); Asia (Georgia); North Africa.

Stenopterus rufus Linnaeus, 1767

Recorded: Cocoş Monastery (Montandon, 1887; Serafim, 2009); Greci (Montandon, 1908; Serafim, 2009); Comarova Forest (Mangalia) (Negru \& Roşca, 1967); Agigea (Serafim \& Maican, 2004); Nifon, Murfatlar, Esechioi, Hagieni Forest, Dumbrăveni Forest, Olimp resort (North Mangalia) (Serafim, 2009); Măcin Mountains National Park (Greci) (Skolka et al., 2006-2007).

Distribution: Europe (except North and Northeast); Asia (Azerbaijan, Armenia, Georgia, Turkmenistan). 
Leiopus nebulosus nebulosus (Linnaeus, 1758)

Recorded: Sulina (Negru, 1968 a; Serafim, 2010); Măcin Mountains National Park (Valea Fagilor Forest) (Skolka et al., 2006-2007).

Distribution: Europe; Asia (Kazakhstan).

Tribe Acanthoderini J. Thomson, 1860

Aegomorphus clavipes (Schrank, 1781)

Recorded: C.A. Rosetti (Serafim, 1993); Canaraua Fetii (Băneasa), Sfântu Gheorghe (Serafim, 2010)

Distribution: Europe; Asia; North Africa.

Tribe Agapanthiini Mulsant, 1839

Agapanthia (Agapanthia) violacea (Fabricius, 1775)

Recorded: Cernavodă (Fleck, 1905 b); Babadag Forest, Techirghiol, Esechioi (Panin \& Săvulescu, 1961); Sălcioara (6 Martie) Forest (Jurilovca) (Serafim, 1993); Letea Forest (Serafim, 1993, 2010); Comarova Forest (Mangalia) (Serafim \& Maican, 2004); Dobrogea (without other data) (Skolka et al., 2005); Oltina, Southern Dobrogea, Esechioi, Canaraua Fetii (Băneasa), Hagieni, Dumbrăveni Forest, Adamclisi, Iortmac Lake, Cocoș Monastery, Negureni Forest, Ciucurova, Valea Teilor, Telița, Revărsarea (Serafim, 2010).

Distribution: Europe (except Iceland, Ireland, Sweden); Asia (Turkey, Azerbaijan, Armenia, Georgia, Russia - East Siberia, Kazakhstan).

Agapanthia (Epoptes) dahli (Richter, 1821)

Recorded: Mangalia (Fleck, 1905 b; Negru \& Roşca, 1967); Caraorman (Ieniştea, 1974; Serafim et al., 2004); Canaraua Fetii (Băneasa) (Balog, 1998; Serafim, 2005); Comarova Forest (Mangalia), Agigea, Olimp resort (North Mangalia), Tuzla (Serafim \& Maican, 2004; Serafim, 2010); Dobrogea (without other data) (Skolka et al., 2005); Valu lui Traian, Babadag Forest, Techirghiol, Hagieni Forest, Chilia Veche, Periprava, Dumbrăveni Forest, Sfântu Gheorghe, Ciucurova, Esechioi, Albeşti, Cheia, Popina Island (Razim Lake) (Serafim, 2010); C.A. Rosetti (Serafim \& Maican, 2011).

Distribution: Europe (except North); Asia.

Agapanthia (Epoptes) kirbyi (Gyllenhal, 1817)

Recorded: Măcin (Montandon, 1908); Canaraua Fetii (Băneasa) (Panin \& Săvulescu, 1961; Săvulescu \& Popescu-Gorj, 1964; Serafim, 2010); Măcin Mountains National Park (Măcin, Greci, Pricopan) (Skolka et al., 2006-2007).

Distribution: Europe (Balkan Peninsula, France, Hungary, Italy, Republic of Moldova, Portugal, South Russia, Slovakia, Spain, Ukraine); Asia (Iran, Israel, Syria, Azerbaijan, Armenia, Georgia, Turkmenistan).

$$
\text { Agapanthia (Epoptes) villosoviridescens (De Geer, 1775) }
$$

Recorded: Mangalia (Montandon, 1906; Panin \& Săvulescu, 1961); Babadag Forest (Panin \& Săvulescu, 1961; Serafim, 2010); Comarova Forest (Mangalia) (Negru \& Roşca, 1967; Serafim \& Maican, 2004; Serafim, 2010); Caraorman (Ieniştea, 1974; Serafim, 2010); Chilia Veche (Serafim, 1993); Periprava (Serafim, 1993, 2010); Canaraua Fetii (Băneasa) (Balog, 1998; Serafim, 2010); Constanța (Palas), Agigea, Neptun resort (North Mangalia) (Serafim \& Maican, 2004; Serafim, 2010); Dobrogea (without other data) (Skolka et al., 2005); Esechioi, Oltina, Dumbrăveni Forest, Hagieni Forest, Southern Dobrogea, Negureni Forest, Dumbrăveni Forest, Celic Dere, Valea Teilor (Serafim, 2010); Măcin Mountains National Park (Chediu canyon) (Skolka et al., 2006-2007).

Distribution: Europe; Asia (East and West Siberia, Georgia, Kazakhstan, Mongolia, Korea). 
Agapanthiola leucaspis (Steven, 1817)

Recorded: Babadag (Fleck, 1905 b); Măcin (Montandon, 1906); Eforie Sud, Valu lui Traian (Panin \& Săvulescu, 1961); Comarova Forest (Mangalia) (Negru \& Roşca, 1967); Letea Forest (Ieniştea, 1968); Periprava (Serafim, 1993, 2010); Canaraua Fetii (Băneasa) (Balog, 1998; Serafim, 2010); Agigea, Comarova Forest (Mangalia), Neptun resort (North Mangalia) (Serafim \& Maican, 2004); Dobrogea (without other data) (Skolka et al., 2005); Măcin Mountains National Park (Măcin, Greci, Pricopan) (Skolka et al., 2006-2007); Dobrogea, Murfatlar, Esechioi, Hagieni Forest, C.A. Rosetti, Dumbrăveni Forest, Niculiţel, Caraorman, Letea Forest (Serafim, 2010).

Distribution: Europe (Austria, Balkan Peninsula, Hungary, Republic of Moldova, Central and South Russia, Slovakia, Ukraine); Asia (Georgia, Russia - East and West Siberia, Central Asia).

\section{Calamobius filum (Rossi, 1790)}

Recorded: Eforie Sud (Panin \& Săvulescu, 1961); Letea Forest (Haşmacul Mare) (Ieniştea, 1968); the littoral of the Black Sea, without collecting data (Gomoiu \& Skolka, 1998); Comarova Forest (Mangalia) (Serafim \& Maican, 2004); Agigea (Serafim \& Maican, 2004; Serafim, 2010; Serafim \& Maican, 2011); Dobrogea (without other data) (Skolka et al., 2005).

Distribution: Europe (except North and North-East); Asia (Near East); North Africa.

Tribe Apodasyini Lacordaire, 1872

Anaesthetis testacea (Fabricius, 1781)

Recorded: Letea Forest (Haşmacurile Mici) (Negru, 1968 a); Dobrogea (without other data) (Skolka et al., 2005); Măcin, C.A. Rosetti (Serafim, 2010).

Material: Dunavăţ, 9.VI.1994.

Distribution: Europe (except North); Asia (Turkey, Kazakhstan).

Deroplia genei genei (Aragona, 1830)

Recorded: Greci (Montandon, 1908); Iortmac Lake (Panin \& Săvulescu, 1961); Canaraua Fetii (Săvulescu \& Popescu-Gorj, 1964); Oltina (Negru \& Roşca, 1967); South Dobrogea, Fântâna Mare (Başpunar) (Serafim, 2010); Măcin Mountains National Park (Măcin, Greci, Pricopan) (Skolka et al., 2006-2007).

Material: Oltina, 15.V.1958.

Distribution: South and Central Europe; Near East.

Tribe Dorcadionini Swainson \& Shuckard, 1840

Dorcadion (Carinatodorcadion) aethiops (Scopoli, 1763)

Recorded: Esechioi (Panin \& Săvulescu, 1961; Balaci, 2000; Serafim, 2010); Dobrogea (without other data) (Skolka et al., 2005); Bugeac, Canaraua Fetii (Băneasa), Hagieni Forest (Serafim, 2010).

Distribution: Europe (Albania, Austria, Bulgaria, Croatia, Czech Republic, Greece, Hungary, Macedonia, Republic of Moldova, Slovakia, Ukraine, Serbia and Montenegro).

Dorcadion (Carinatodorcadion) fulvum erythropteron Fischer von Waldheim, 1823 Recorded: Mangalia (Jaquet, 1898 a; Fleck, 1905 b; Montandon, 1908); Dobrogea (without other data) (Skolka et al., 2005); Canaraua Fetii (Băneasa), Dumbrăveni Forest (Serafim, 2010).

Distribution: Europe (Albania, Bosnia Herzegovina, Bulgaria, Croatia, Macedonia, Republic of Moldova, Poland, Ukraine, Serbia and Montenegro).

Dorcadion (Cribridorcadion) axillare Küster, 1847

Recorded: Mangalia (GANMNHB coll.) (Montandon, 1908; Dascălu \& Fusu, 2012); Esechioi, Hagieni, Oltina, Bugeac, Babadag, Constanţa, Histria, (GANMNHB 
coll.); Dobrudja, (NHMW coll.); Mangalia (Mikhail L. Danilevsky coll.) (Dascălu \& Fusu, 2012).

Remarks. This species was mentioned in 1908 from several localities in southeastern Romania by Arnold Lucien Montandon, but this record has been overlooked or misinterpreted by the authors who have published later. The species was cited as Dorcadion arenarium var. axillare. Based on all published data, the specimens in museum collections and some recently collected Dascălu and Fusu (2012) have shown that the species $D$. axillare is in fact widely distributed in Romania. So, the presence of this species is confirmed in Romania more than one hundred years after the last published record. The species was commonly considered endemic for Bulgaria. The specimens from the northern part of Romania differ from those in Bulgaria and are considered to belong to a different subspecies, $D$. axillare moldavicum ssp. nov. The cytogenetic study has revealed a karyotype with $2 \mathrm{n}=24$ and this is the first comprehensive information on the chromosomes of the genus Dorcadion (Dascălu \& Fusu, 2012). According to Dascălu \& Fusu (2012), the specimens of D. axillare from Babadag (Dobruja) were wrongly mentioned as D. pusillum (Panin \& Săvulescu, 1961) and the records from Mangalia (Montandon, 1908; Serafim, 2010) are based on a specimen of $D$. axillare (1 spec., Mangalia, without other data, Montandon coll.)

Distribution: the nominal subspecies is distributed all over Bulgaria and in $\mathrm{S}$ Romania (Banat and Dobrogea).

Dorcadion (Cribridorcadion) equestre transsilvanicum Ganglbauer, 1884

Recorded: Constanţa, Cernavodă, Oltina, Techirghiol (Jaquet, 1898 a; Fleck, 1905 b; Panin \& Săvulescu, 1961); Mangalia (Montandon, 1908; Negru \& Roşca, 1967); Dobrogea (without other data) (Skolka et al., 2005); Oltina (Serafim, 2010).

Distribution: Europe (Republic of Moldova, Romania).

Dorcadion gashtarovi Sama, Dascălu \& Pesarini, 2010

Recorded: Mangalia Lake, Hagieni Forest Nature Reserve, Hagieni village, Târguşor, Babadag Forest, Albeşti (Sama et al., 2010); Esechioi, Popina Island (Razim Lake) (Serafim, 2010).

Distribution: Europe (Romania, Bulgaria).

Remarks. According to Sama et al. (2010) in Romania this species was firstly cited as Dorcadion divisum Germar var. subinterruptum Pic, 1900 by Montandon (1908). The specimens from Hagieni preserved in the Banat Museum collections were published as Dorcadion septemlineatum (Waltl, 1838) by Balaci (2000).

Dorcadion (Cribridorcadion) litigiosum litigiosum (Ganglbauer, 1884)

Recorded: Cernavodă (Fleck, 1905 b; Montandon, 1906); Turcoaia (Igliţa) Lake, Târguşor (Ester) (Montandon, 1906, 1908); Măcin (Montandon, 1908).

Remarks. The record from Babadag, based on a single male, leg. Cârdei (Panin \& Săvulescu, 1961) is more likely connected with $D$. gashtarovi as a specimen collected by the same collector from the same area is in the type series of D. gashtarovi (Dascălu personal communication).

Distribution: Europe (Bulgaria, Republic of Moldova, Romania).

Dorcadion (Cribridorcadion) murrayi (Küster, 1847)

Recorded: Dobrogea (without other data) (Skolka et al., 2005); Periprava, Izvoarele (Serafim, 2010).

Distribution: Europe (Romania, Serbia and Montenegro).

Dorcadion (Cribridorcadion) pedestre pedestre (Poda, 1761)

Recorded: Babadag Forest (Jaquet, 1901; Fleck, 1905 b; Serafim, 2010); Constanţa, Ciucurova, Murfatlar (Fleck, 1905 b); Mangalia (Montandon, 1908); Periprava, Esechioi, Popina Island (Razim Lake) (Serafim, 1993, 2010); Canaraua Fetii 
(Băneasa) (Balog, 1998; Serafim, 2010); Oltina, Esechioi (Balaci, 2000); Dobrogea (without other data) (Skolka et al., 2005); Măcin Mountains National Park (Măcin) (Skolka et al., 2006-2007); Măcin, Greci, Southern Dobrogea, Hagieni Forest, Cocoş Monastery, Enisala, Sălcioara (6 Martie) Forest (Jurilovca), Dumbrăveni Forest, Celic Dere, Revărsarea (Serafim, 2010); Visterna (Serafim \& Maican, 2011).

Distribution: Europe (Albania, Austria, Bulgaria, Croatia, Czech Republic, Hungary, Macedonia, Republic of Moldova, Poland, Romania, Slovakia, Ukraine, Serbia and Montenegro).

Dorcadion (Cribridorcadion) scopolii (Herbst, 1784)

Recorded: Hagieni Forest (Negru \& Roşca, 1967); Sfântu Gheorghe (Serafim, 2010). Distribution: Europe (Bulgaria, Croatia, Czech Republic, Hungary, Republic of Moldova, Poland, Romania, Slovakia, Ukraine, Serbia and Montenegro).

Dorcadion (Cribridorcadion) tauricum (Waltl, 1838)

Recorded: Constanţa (Jaquet, 1898 a); Mangalia (Jaquet, 1898 a; Fleck, 1905 b; Montandon, 1908; Serafim, 2010); Comarova Forest (Mangalia) (Negru, 1957); Valu lui Traian, Babadag Forest (Panin \& Săvulescu, 1961; Serafim, 2010); Canaraua Fetii (Băneasa) (Balog, 1998; Serafim, 2010); Dobrogea (without other data) (Skolka et al., 2005); Esechioi, Southern Dobrogea, Iortmac Lake, Hagieni Forest, Dumbrăveni Forest, Negureni Forest, Celic Dere, Valea Teilor (Serafim, 2010).

Distribution: Europe (Bulgaria, Greece, Romania, Turkey, Ukraine).

Neodorcadion bilineatum (Germar, 1824)

Recorded: Cocoş Monastery (Montandon, 1887, 1906; Serafim, 2010); Mangalia (Jaquet, 1898 a, 1901; Fleck, 1905 b; Montandon, 1908; Negru \& Roşca, 1967; Serafim, 2010); Tulcea, Turcoaia (Iglița) Lake (Jaquet, 1901; Fleck, 1905 b); Constanța (Jaquet, 1901, 1903; Fleck 1905 b; Serafim, 2010); Babadag Forest (Fleck, 1905 b; Serafim, 2010); Măcin (Montandon, 1906); Comarova Forest (Mangalia) (Negru \& Roşca, 1967); Periprava (Ieniştea, 1968); Popina Island (Razim Lake), Enisala, Sălcioara (6 Martie) Forest (Jurilovca) (Serafim, 1993); Canaraua Fetii (Băneasa) (Balog, 1998; Serafim, 2010); Mamaia resort (Serafim et al., 2004); Esechioi, Valu lui Traian, Eforie, Hagieni Forest, Techirghiol, Agigea, Iortmac Lake, Negureni Forest, Dumbrăveni Forest, Ciucurova, Celic Dere, Teliţa, Valea Fagilor Forest near Luncaviţa (Serafim, 2010).

Material: Sarinasuf, 24.VI.1963.

Distribution: Europe (Albania, Bosnia Herzegovina, Bulgaria, Croatia, Greece, Hungary, Macedonia, Republic of Moldova, Romania, Slovakia, Ukraine, Serbia and Montenegro).

\section{Neodorcadion exornatum (Frivaldsky von Frivald, 1835)}

Recorded: Cocoş Monastery (Montandon, 1887); Măcin (Fleck, 1905 b); Cernavodă (Montandon, 1906); Mangalia (Montandon, 1908); Canaraua Fetii (Băneasa), Esechioi, Iortmac Lake (Panin \& Săvulescu, 1961; Serafim, 2010), Hagieni Forest, Babadag Forest (Serafim, 2010).

Distribution: Europe (Bulgaria, Greece, Romania, Turkey); Asian Turkey.

Remarks. In the old papers the species is cited as Neodorcadion balcanicum Tournier, 1872.

Tribe Lamiini Latreille, 1825

Morimus asper funereus Mulsant, 1863

Recorded: Isaccea (Montandon, 1887, 1906); Turcoaia (Iglița) Lake, Pricopan (Fleck, 1905 b); Ciucurova (Fleck, 1905 b; Serafim, 2010); Greci (Montandon, 1908); Canaraua Fetii (Băneasa) (Panin \& Săvulescu, 1961; Serafim, 2010); Caraorman (Ieniştea, 1974); Southern Dobrogea, Cocoş Monastery, Sălcioara (6 Martie) Forest 
(Jurilovca), Slava Rusă, Celic Dere, Valea Fagilor Forest near Luncaviţa (Serafim, 2010); Babadag Forest (Serafim, 2010; Serafim \& Maican, 2011); Măcin Mountains National Park (Măcin, Greci) (Skolka et al., 2006-2007); Delta Dunării, North Dobrogea Plateau (Tatole et al., 2009).

Distribution: Europe (Austria, Balkan Peninsula, Czech Republic, Hungary, Italy, Republic of Moldova, Slovakia, Ukraine).

Tribe Mesosini Mulsant, 1839

Mesosa (Aplocnemia) nebulosa nebulosa (Fabricius, 1781)

Recorded: Pricopan (Fleck, 1905 b; Panin \& Săvulescu, 1961; Skolka et al., 20062007); Southern Dobrogea, Babadag Forest, Dumbrăveni Forest, Luncaviţa (Serafim, 2010); Măcin Mountains National Park (Măcin, Greci) (Skolka et al., 2006-2007).

Distribution: Europe; Asia (Azerbaijan, Armenia, Georgia, Asian Turkey).

Mesosa (Mesosa) curculionoides (Linnaeus, 1761)

Recorded: Greci (Montandon, 1908); Constanța (Panin \& Săvulescu, 1961); C.A. Rosetti (Negru, 1968 a; Serafim 2010), Babadag Forest, Letea Forest, Sulina, Valea Fagilor Forest near Luncaviţa (Serafim, 2010).

Distribution: Europe; Asia (Iran, Turkey, Kazakhstan, Northern and Southwestern China).

Tribe Phytoeciini Mulsant, 1839

Coptosia albovittigera Heyden, 1863

Recorded: Valu lui Traian (Panin \& Săvulescu, 1961 as Coptosia bithynensis Ganglbauer); Serafim (2010) gives this species as Coptosia sp. based on the same specimen collected by Nicolae Săvulescu.

Distribution: Bulgaria, Greece, Macedonia, European and Asian Turkey.

Oberea (Amaurostoma) erythrocephala erythrocephala (Schrank, 1776)

Recorded: Mangalia, Techirghiol (Fleck, 1905 b); Măcin (Fleck, 1905 b; Skolka et al., 2006-2007); Turcoaia (Igliţa) Lake (Montandon, 1906); Caraorman (Ieniştea, 1974); Danube Delta, Sfântul Gheorghe arm - Buhaz sand bank (Serafim, 1993); Agigea (Serafim \& Maican, 2004; Serafim, 2010); Valu lui Traian, Canaraua Fetii (Băneasa), Sfântu Gheorghe, Hagieni Forest, C.A. Rosetti, Southern Dobrogea, Popina Island (Razim Lake) (Serafim, 2010); Măcin Mountains National Park (Greci, Pricopan) (Skolka et al., 2006-2007).

Distribution: Europe (except North); Asia (Near East, Siberia, Kazakhstan, China); Oriental Region.

Oberea (Amaurostoma) euphorbiae (Germar, 1813)

Recorded: Mangalia (Fleck, 1905 b; Negru \& Roşca, 1967); Turcoaia (Igliţa) Lake (Montandon, 1906); Băneasa (Negru \& Roşca, 1967); C.A. Rosetti (Panin \& Săvulescu, 1961; Serafim \& Maican, 2011); Periprava (Negru, 1968 a; Serafim, 1993, 2010); Caraorman, Maliuc - Mila 26 (Serafim, 1993, 2010); Danube Delta, Sfântul Gheorghe arm - Buhaz sand bank, Agigea, Letea Forest, Sfântu Gheorghe, Dunavăţ (Serafim, 2010); Măcin Mountains National Park (Măcin, Greci, Pricopan) (Skolka et al., 2006-2007).

Distribution: Europe (Austria, Bulgaria, Croatia, Czech Republic, Germany, Greece, Hungary, Italy, Republic of Moldova, Central and South Russia, Slovakia, Switzerland, Ukraine, Serbia and Montenegro); Asia (Armenia, Kazakhstan, Russia - West Siberia).

Oberea (Oberea) linearis (Linnaeus, 1761)

Recorded: Caraorman (Serafim, 1993); C.A. Rosetti (Serafim \& Maican, 2011).

Distribution: Europe; Near East (Turkey). 
Oberea (Oberea) oculata (Linnaeus, 1758)

Recorded: Mangalia, Techirghiol (Fleck, 1905 b); Periprava (Serafim, 1993, 2010);

Comarova Forest (Mangalia), Sfântu Gheorghe (Serafim, 2010).

Distribution: Europe; Asia; North Africa; Oriental Region.

Oberea (Oberea) pedemontana Chevrolat, 1856

Recorded: Hagieni Forest (Negru \& Roşca, 1967; Balaci, 2000; Serafim, 2010); Murfatlar (Serafim, 2010).

Distribution: Europe (Austria, Hungary, Italy, Balkan Peninsula); Near East.

Opsilia coerulescens (Scopoli, 1763)

Recorded: Techirghiol (Fleck, 1905 b); Mangalia (Fleck, 1905 b; Negru \& Roşca, 1967); Canaraua Fetii (Băneasa) (Balog, 1998; Serafim, 2010); Comarova Forest (Mangalia), Agigea (Serafim \& Maican, 2004; Serafim, 2010); Greci, Southern Dobrogea, Hagieni Forest, Esechioi, Slava Rusă, Babadag Forest, Celic Dere Monastery (Serafim, 2010).

Distribution: Europe (except North-West); Asia (Near East, West Siberia, Kazakhstan, Uzbekistan, China); North Africa.

Opsilia uncinata (Redtenbacher, 1842)

Recorded: Dobrogea (Panin \& Săvulescu, 1961).

Distribution: Europe (except North); Asia (Turkey, Turkmenistan).

Phytoecia (Cardoria) scutellata (Fabricius, 1792)

Recorded: Dobrogea (without other data) (Skolka et al., 2005); Hagieni Forest (Serafim, 2010).

Distribution: Europe (Austria, Czech Republic, Germany, Greece, Hungary, Republic of Moldova, South Russia, Slovakia, Ukraine); Asia (Turkey, the Caucasian Republics, Iran).

\section{Phytoecia (Helladia) praetextata praetextata (Steven, 1817)}

Recorded: Esechioi (Panin \& Săvulescu, 1961; Săvulescu \& Popescu-Gorj, 1964; Balaci, 2000; Serafim, 2010).

Distribution: Europe (Bulgaria, Romania, Russia - South European Territory, Ukraine); Asia (Azerbaijan, Armenia, Georgia, Turkey, Syria).

Phytoecia (Phytoecia) caerulea caerulea (Scopoli, 1772)

Recorded: Mangalia, Babadag Forest, Măcin (Fleck, 1905 b; Negru \& Roşca, 1967; Serafim, 2010); Turcoaia (Igliţa) Lake (Montandon, 1906); Dobrogea (Panin \& Săvulescu, 1961); C.A. Rosetti, Doloşman Cape (Razim Lake), Maliuc - Mila 26 (Serafim, 1993, 2010); Comarova Forest (Mangalia), Agigea (Serafim \& Maican, 2004; Serafim, 2010); Oltina, Valu lui Traian, Esechioi, Canaraua Fetii (Băneasa), Hagieni Forest, Dumbrăveni Forest, Iortmac Lake, Celic Dere (Serafim, 2010); Visterna (Serafim \& Maican, 2011); Măcin Mountains National Park (Măcin) (Skolka et al., 2006-2007).

Distribution: Europe (Balkan Peninsula, Iberian Peninsula, Austria, Belarus, Czech Republic, Hungary, Italy, Republic of Moldova, Central and South Russia, Sicily, Slovakia, Ukraine); Asia (Azerbaijan, Armenia, Georgia, Turkey, Syria, Iran, Turkmenistan, Uzbekistan).

Remarks. Reported as Saperda rufimana Schrank, 1789.

$$
\text { Phytoecia (Phytoecia) cylindrica (Linnaeus, 1758) }
$$

Recorded: Oltina (Negru \& Roşca, 1967; Serafim, 2010), Canaraua Fetii (Băneasa), Comarova Forest (Mangalia), Agigea, Dumbrăveni Forest, Hagieni Forest, Negureni Forest, Valea Fagilor Forest, Luncaviţa (Serafim, 2010).

Distribution: Europe; Asia (Turkey, Iran, East and West Siberia, the Caucasian Republics, Kazakhstan). 
Phytoecia (Phytoecia) icterica (Schaller, 1783)

Recorded: Canaraua Fetii (Băneasa) (Balog, 1998; Serafim, 2010); Oltina, Esechioi, Dumbrăveni Forest, Southern Dobrogea, Negureni Forest, Negureni (Valea Cişmelelor) (Serafim, 2010).

Distribution: Europe (Austria, Balkan Peninsula, Czech Republic, France, Germany, Hungary, Italy, Republic of Moldova, Poland, Portugal, Central and South Russia, Slovakia, Slovenia, Spain, Switzerland); Asia (Kazakhstan, West Siberia).

Phytoecia (Phytoecia) nigricornis (Fabricius, 1781)

Recorded: Măcin (Montandon, 1906, 1908); Comarova Forest (Mangalia) (Negru \& Roşca, 1967; Serafim \& Maican, 2004); Canaraua Fetii (Băneasa), Esechioi, Celic Dere Monastery, Valea Teilor, Ciucurova, Teliţa, Dumbrăveni Forest - Ceair Valley (Serafim, 2010); Măcin Mountains National Park (Măcin, Greci, Pricopan) (Skolka et al., 2006-2007).

Distribution: Europe; Asia (the Caucasian Republics, East and West Siberia, Kazakhstan).

Phytoecia (Phytoecia) pustulata pustulata (Schrank, 1776)

Recorded: Pricopan (Fleck, 1905 b); Canaraua Fetii (Băneasa) (Balog, 1998); Măcin Mountains National Park (Măcin, Greci, Pricopan) (Skolka et al., 2006-2007); Valu lui Traian, Hagieni, Esechioi (Serafim, 2010).

Distribution: Europe (except North); Asia (Near East, Central Asia).

Phytoecia (Phytoecia) virgula (Charpentier, 1825)

Recorded: Strunga (Oltina) (Negru \& Roșca, 1967); Cogeacu Bandiţi (Negru \& Roşca, 1967; Serafim, 2010); Agigea (Serafim \& Maican, 2004; Serafim, 2010; Serafim \& Maican, 2011); Dobrogea, Măcin, Dobromir, Murfatlar, Bugeac Lake, Valu lui Traian, Oltina, Hagieni Forest, Canaraua Fetii (Băneasa), Esechioi, Dumbrăveni Forest, Adamclisi, Negureni Forest, Izvoarele, Ciucurova Forest, Valea Teilor, Valea Fagilor Forest near Luncaviţa, Cerna, Suluc Valley (Serafim, 2010); Măcin Mountains National Park (Măcin, Greci, Pricopan) (Skolka et al., 2006-2007; Serafim, 2010).

Distribution: Europe (except North), Asia. Pilemia hirsutula hirsutula (Frolich, 1793)

Recorded: Dobrogea, Hârşova, Valu lui Traian, Comarova Forest (Mangalia), Hagieni Forest, Dumbrăveni Forest (Serafim, 2010).

Distribution: Europe (Albania, Hungary, Balkan Peninsula, Slovakia, Ukraine); Asia (Near East, West Siberia).

\section{Pilemia tigrina Mulsant, 1851}

Recorded: Dumbrăveni Forest (Serafim, 2010).

Distribution: Europe (Bulgaria, Hungary, Romania, Russia - South European Territory, Ukraine, Serbia and Montenegro); Asia (Armenia).

Tribe Pogonocherini Mulsant, 1839

Exocentrus adspersus Mulsant, 1846

Recorded: Greci (Montandon, 1887; Skolka et al., 2006-2007; Serafim, 2010); Măcin (Montandon, 1906); Măcin Mountains National Park (Măcin) (Skolka et al., 20062007); Babadag Forest (Serafim, 2010).

Distribution: Europe (except North); Asia (The Caucasian Republics, Turkey, Syria). Exocentrus lusitanus (Linnaeus, 1767)

Recorded: Măcin Mountains National Park (Măcin, Greci) (Montandon, 1906, 1908; Skolka et al., 2006-2007); Canaraua Fetii (Băneasa) (Serafim, 2010).

Distribution: Europe; Asia (the Caucasian republics, East end West Siberia, Kazakhstan). 
Exocentrus punctipennis Mulsant \& Guillebeau, 1856

Recorded: Greci (Montandon, 1887, 1908); Măcin (Montandon, 1906); Periprava (Panin \& Săvulescu, 1961; Serafim, 2010); Canaraua Fetii (Băneasa), Letea Forest, Babadag Forest (Serafim, 2010); Măcin Mountains National Park (Măcin, Greci) (Skolka et al., 2006-2007).

Distribution: Europe (except North), the Caucasus.

Pogonocherus hispidus (Linnaeus, 1758)

Recorded: Greci (Montandon, 1908); Hagieni (Negru \& Roşca, 1967); Dumbrăveni Forest, Furnica (Serafim, 2010); Măcin Mountains National Park (Măcin, Greci, Pricopan) (Skolka et al., 2006-2007).

Distribution: Europe, Near East, North Africa.

Tribe Saperdini Mulsant, 1839

Saperda carcharias (Linnaeus, 1758)

Recorded: Maliuc - Mila 26 (Serafim, 1993).

Distribution: Europe, Near East, Central Asia, East Palaearctic, Oriental Region.

Saperda octopunctata (Scopoli, 1772)

Recorded: Babadag Forest, Ciucurova Forest (Serafim, 2010); Măcin Mountains National Park (Măcin, Cetăţuia, Valea Seacă) (Skolka et al., 2006-2007).

Distribution: Europe (except North), Near East.

Saperda perforata (Pallas, 1773)

Recorded: Letea Forest (Haşmacurile Mici) (Negru, 1968 a); Periprava (Balaci, 2000; Serafim, 2010); Letea Forest, Babadag Forest, C.A. Rosetti (Serafim, 1993, 2010); Babadag Forest (Serafim, 2010).

Distribution: Europe; Asia; North Africa (Algeria).

Saperda populnea (Linnaeus, 1758)

Recorded: Danube Delta (Panin \& Săvulescu, 1961; Săvulescu, 1985); Sfântu Gheorghe, Canaraua Fetii (Băneasa), Dumbrăveni Forest, Furnica (Serafim, 2010). Distribution: Europe; Asia; Nearctic Region.

Saperda punctata (Linnaeus, 1767)

Recorded: Babadag Forest, Canaraua Fetii (Băneasa), Hagieni Forest (Serafim, 2010).

Distribution: Europe; Asia (the Caucasian republics, Turkey, Cyprus); North Africa (Algeria).

Saperda scalaris scalaris (Linnaeus, 1758)

Recorded: Dobrogea, without other data (Serafim, 2010).

Distribution: Europe; Asia (the Caucasian republics, Turkey, Kazakhstan); North Africa (Algeria).

Stenostola ferrea ferrea (Schrank, 1776)

Recorded: Pricopan (Fleck, 1905 b); Eforie Sud (Carmen Sylva) (Serafim, 2010); Măcin Mountains National Park (Măcin, Greci, Pricopan) (Skolka et al., 2006-2007). Distribution: Europe (except North); Near East.

Tribe Tetropini Portevin, 1927

Tetrops praeustus praeustus (Linnaeus, 1758)

Recorded: Comarova Forest (Mangalia) (Negru, 1957, 1965; Negru \& Roşca, 1967); Tulcea (Serafim, 1993, 2010); Canaraua Fetii (Băneasa), Constanţa (Palas), Hagieni Forest, Tulcea (Serafim, 1993, 2010); Babadag Forest (Serafim \& Maican, 2011).

Distribution: Europe; Asia (Near East, Mongolia, East and West Siberia). 
Family Orsodacnidae C. H. Thomson, 1859

Orsodacne cerasi (Linnaeus, 1758)

Recorded: Pricopan (Fleck, 1905 b); Beştepe, Agighiol, Iancina Cape (Crişan, 1994); Dobrogea (without other data) (Skolka et al., 2005); Măcin, Greci, Pricopan (Skolka et al., 2006-2007).

Distribution: from Great Britain and S France to W Siberia, S Scandinavia, Asia Minor.

\section{Orsodacne humeralis Latreille, 1804}

(syn: lineola Panzer, 1795)

Recorded: Cernavodă (Hurmuzachi, 1904); Hagieni (Negru, 1957); Lupilor sand bank (Sinoe Lake) (Crişan, 1995 a); Dobrogea (without other data) (Skolka et al., 2005); Romanian Dobrogea (Gruev, 2005).

Distribution: France, S part of Central Europe, Italy, Balkan Peninsula, Asia Minor.

Family Chrysomelidae Latreille, 1802

Subfamily Donaciinae Kirby, 1837

Tribe Donaciini Kirby, 1837

Donacia cinerea Herbst, 1784

Recorded: Maliuc (Ieniştea, 1968); Caraorman (Ieniştea, 1974); Doloşman Cape (Razim Lake) (Crişan, 1993); Dunavăț, Popina Island (Razim Lake) (Crişan, 1995 a); Dobrogea (without other data) (Skolka et al., 2005).

Material: Gura Portiţei, 4.VI.1994.

Distribution: from S France and Ireland to Altai.

Donacia crassipes Fabricius, 1775

Recorded: Fortuna Lake, Crişan (Ieniştea, 1968); Caraorman sand bank (Ieniştea, 1974); Letea Forest (Crişan, 1994); Dobrogea (without other data) (Skolka et al., 2005).

Distribution: from Ireland to E Siberia; rare in S Europe.

Donacia dentata Hoppe, 1795

Recorded: Mangalia (Negru \& Roşca, 1967); Sulina (Negru, 1968 a); at the intersection of Sulina arm with Magearu Channel (Crişan, 1994); Dobrogea (without other data) (Skolka et al., 2005); Romanian Dobrogea (Gruev, 2005).

Distribution: from Ireland and France to Altai.

Donacia impressa Paykull, 1799

Recorded: Romanian Dobrogea (Gruev, 2005).

Distribution: from Iberian Peninsula and Ireland to Mongolia.

Donacia marginata Hoppe, 1795

Recorded: Periprava, Sulina (Negru, 1968 a); Caraorman sand bank (Ieniştea, 1974).

Distribution: North Africa, Europe, Central Asia.

Donacia simplex Fabricius, 1775

Recorded: Maliuc (Crişan, 1993); Lupilor sand bank (Sinoe Lake) (Crişan, 1995 a);

Dobrogea (without other data) (Skolka et al., 2005).

Distribution: from the British Isles and Spain to Mongolia and Siberia.

Donacia thalassina thalassina Germar, 1811

Recorded: Caraorman sand bank (Ieniştea, 1974).

Distribution: Europe, Siberia, NW China, Japan.

Donacia tomentosa Ahrens, 1810

Recorded: C.A. Rosetti (Negru, 1968 a). 
Distribution: from S France to Siberia.

Donacia vulgaris vulgaris Zschach, 1788

Recorded: C.A. Rosetti (Negru, 1968 a).

Distribution: transpalaearctic species, distributed from the British Isles and Spain to Japan.

Tribe Haemoniini S.-H. Chen, 1941

Macroplea mutica (Fabricius, 1792)

Recorded: Mamaia (Hoinic, 1994).

Distribution: the coast of the N Baltic, Mediterranean and Caspian Sea.

Tribe Plateumarini Böving, 1922

Plateumaris (Plateumaris) braccata (Scopoli, 1772)

Recorded: Comarova Forest (Mangalia), Mangalia, Agigea, Neptun resort (Serafim \& Maican, 2004).

Distribution: W Palaearctic species, distributed from Ireland and Spain to Turkistan.

Plateumaris (Plateumaris) rustica (Kunze, 1818)

Recorded: Agigea (Serafim \& Maican, 2004).

Distribution: W Palaearctic species, distributed from Algeria to W Siberia.

Subfamily Criocerinae Latreille, 1804

Crioceris asparagi (Linnaeus, 1758)

Recorded: Măcin (Montandon, 1908); Măcin Mountains, Greci, Pricopan (Skolka et al., 2006-2007).

Distribution: from Iberian Peninsula to Central Asia; introduced in North America, Argentina and Tanzania.

\section{Crioceris duodecimpunctata (Linnaeus, 1758)}

Recorded: Greci (Montandon, 1887); Măcin (Montandon, 1906); Hagieni (Negru \& Roşca, 1967); Periprava, C.A. Rosetti (Negru, 1968 a); Caraorman sand bank (Ieniştea, 1974; Crişan, 1993); Letea Forest (Crişan, 1994); Olimp resort (North Mangalia) (Serafim \& Maican, 2004); Dobrogea (without other data) (Skolka et al., 2005); Romanian Dobrogea (Gruev, 2005); Măcin, Greci, Pricopan (Skolka et al., 2006-2007); Visterna, Babadag (Serafim \& Maican, 2011).

Distribution: transpalaearctic species, distributed from Portugal and England to Korea, in N Africa and in Fennoscandia absent.

Crioceris quatuordecimpunctata (Scopoli, 1763)

Recorded: Greci (Montandon, 1887); Măcin (Montandon, 1906); Periprava, C.A. Rosetti (Negru, 1968 a); Caraorman sand bank (Ieniştea, 1974; Crişan, 1993); Letea Forest (Crişan, 1994); Dobrogea (without other data) (Skolka et al., 2005); Romanian Dobrogea (Gruev, 2005); Măcin, Greci, Pricopan (Skolka et al., 2006-2007).

Distribution: SE Europe, Central Europe, Central Asia.

$$
\text { Crioceris quinquepunctata (Scopoli, 1763) }
$$

Recorded: Letea Forest (Haşmacul Mare) (Ieniştea, 1968); Letea Forest (Crişan, 1994); Dobrogea (without other data) (Skolka et al., 2005).

Distribution: basins of the Danube, Dnieper and Volga rivers.

Lema (Lema) cyanella (Linnaeus, 1758)

Recorded: Periprava (Ieniştea, 1968); Maliuc, Gorgova (Crişan, 1993); Dobrogea (without other data) (Skolka et al., 2005).

Material: 3 specs, Periprava, 13.V.1964, leg. Xenia Scobiola Palade; 1 spec., C.A. Rosetti, 20.VII.1964, leg. Xenia Scobiola Palade; 1 spec., Canaraua Fetii, 18.IX.1964, leg. Ştefan Negru; 2 specs, Hagieni Forest, 29.VI.1973, leg. Aurelian Popescu-Gorj; 
8 specs, Cocoş Monastery, 1.VII.1975, leg. Aurelian Popescu-Gorj; Dumbrăveni Forest, 18.V.1994.

Distribution: transpalaearctic species, distributed from Spain and the British Isles to Korea.

\section{Lilioceris faldermanni (Guérin-Méneville, 1844)}

Recorded: Romanian Dobrogea (Gruev, 2005).

Distribution: Balkan Peninsula, Asia Minor, Syria, Caucasian countries, Central Asia.

\section{Lilioceris lilii (Scopoli, 1763)}

Material: Canaraua Fetii (Băneasa), Negureni Forest, 17-19.V.1993, leg. Mihaela Barcan.

Distribution: widely distributed from Morocco and Great Britain to E Asia.

\section{Lilioceris merdigera (Linnaeus, 1758)}

Recorded: Comarova Forest (Mangalia) (Negru \& Roşca, 1967); C.A. Rosetti (Negru, 1968 a); Cocoş Monastery (Roşca, 1976); Dobrogea (without other data) (Skolka et al., 2005); Romanian Dobrogea (Gruev, 2005).

Distribution: transpalaearctic species, distributed from Iberian Peninsula, France and S Norway to Japan.

Oulema erichsonii (Suffrian, 1841)

Material: 3 specs, Cocoş Monastery, 1.VII.1975, leg. Aurelian Popescu-Gorj.

Distribution: Central and N Europe, from Spain and Ireland to Finland, N Russia, basin of Volga.

Oulema gallaeciana (Heyden, 1870)

Recorded: Romanian Dobrogea (Gruev, 2005).

Distribution: N, W and Central part of Europe, basin of the Danube, European Russia, W Siberia, N part of Central Asia.

Oulema melanopus (Linnaeus, 1758)

Recorded: Mangalia (Montandon, 1887, 1906); Măcin (Fleck, 1905 b); Comarova Forest (Mangalia) (Montandon, 1906; Negru, 1957; Negru \& Roşca, 1967); Greci (Montandon, 1908); Babadag (Crişan, 1993); Beştepe (Crişan, 1994); Canaraua Fetii (Băneasa) (Balog, 1998); Agigea Natural Reserve (Serafim \& Maican, 2004); Romanian Dobrogea (Gruev, 2005); Măcin, Greci, Pricopan (Skolka et al., 20062007).

Material: 2 specs, Mangalia, leg. Eduard Fleck; 6 specs, Niculițel, 21-28.VI.1967, leg. Medeea Weinberg, Xenia Scobiola Palade.

Distribution: W Palaearctic species, distributed from Morocco, Ireland and S Norway to Near East, Central Siberia and Mongolia.

Oulema tristis (Herbst, 1786)

(syn: flavipes Suffrian, 1841)

Recorded: Greci (Montandon, 1908); Caraorman (Crişan, 1993); Dobrogea (without other data) (Skolka et al., 2005); Romanian Dobrogea (Gruev, 2005); Măcin, Greci, Pricopan (Skolka et al., 2006-2007).

Material: 1 spec., Periprava, 8.X.1963, leg. Xenia Scobiola Palade; 3 specs, Periprava, 13.V.1964, leg. Medeea Weinberg.

Distribution: from E France to Japan. 
Cassida atrata Fabricius, 1787

Recorded: Mangalia (Montandon, 1887, 1908; Fleck, 1905 b; Negru \& Roşca, 1967); Dobrogea (without other data) (Skolka et al., 2005); Romanian Dobrogea (Gruev, 2005).

Material: 3 specs, Murfatlar, 4.X.1951, 29.IX.-4.X.1951, leg. Nicolae Săvulescu.

Distribution: SE Europe and Asia Minor.

Cassida canaliculata Laicharting, 1781

Recorded: Cernavodă (Fleck, 1905 b).

Distribution: from E France to Kazakhstan, basin of Danube and Ukraine.

Cassida denticollis Suffrian, 1844

Recorded: Chilia Veche (Ieniştea, 1968); Maliuc, Caraorman (Crişan, 1993); Dobrogea (without other data) (Skolka et al., 2005).

Material: Canaraua Fetii, 19.V.1993; Dumbrăveni Forest, 18.V.1994.

Distribution: from the British Isles to Mongolia.

Cassida ferruginea Goeze, 1777

Recorded: Babadag (Montandon, 1887; Fleck, 1905 b); Constanţa (Fleck, 1905 b); Măcin Mountains (Montandon, 1906; Roşca, 1976; Skolka et al., 2006-2007); Romanian Dobrogea (Gruev, 2005).

Material: 5 specs, Măcin, leg. Arnold Lucien Montandon; 1 spec., Mangalia, leg. Arnold Lucien Montandon.

Distribution: from Pyrenees and N France to Mid Siberia.

Cassida murraea murraea Linnaeus, 1767

Recorded: Caraorman (Crişan, 1993); Lupilor sand bank (Sinoe Lake) (Crişan, 1995 a); Dobrogea (without other data) (Skolka et al., 2005).

Distribution: transpalaearctic species, distributed from the British Isles to Japan. Cassida nebulosa Linnaeus, 1758

Recorded: Mangalia (Fleck, 1905 b; Negru \& Roşca, 1967); Constanţa, Orliga, Turcoaia (Igliţa) Lake (Fleck, 1905 b); Greci (Montandon, 1908); Babadag (Crişan, 1993); Beştepe, Agighiol (Crişan, 1994); Sfântu Gheorghe, Popina Island (Razim Lake) (Crișan, 1995 a); Dobrogea (without other data) (Skolka et al., 2005); Romanian Dobrogea (Gruev, 2005); Cetăţuia (Skolka et al., 2006-2007).

Material: 1 spec., Babadag, leg. Eduard Fleck; 1 spec., Canaraua Fetii (Băneasa), 20.V.1994.

Distribution: transpalaearctic species, distributed from the British Isles to Japan; introduced also in North America.

Cassida nobilis Linnaeus, 1758

Recorded: Constanţa (Montandon, 1887; Fleck, 1905 b); Gorgova, Maliuc (Crişan, 1993); Dobrogea (without other data) (Skolka et al., 2005).

Material: 2 specs, Cernavodă, Măcin, leg. Arnold Lucien Montandon.

Distribution: transpalaearctic species, distributed from Ireland to Japan.

Cassida prasina Illiger, 1798

Recorded: Maliuc (Crişan, 1993); Beștepe (Crişan, 1994); Dobrogea (without other data) (Skolka et al., 2005); Romanian Dobrogea (Gruev, 2005).

Material: Dumbrăveni Forest, 18.V.1994.

Distribution: from Pyrenees, Great Britain and S Sweden to W China.

Cassida rubiginosa rubiginosa $\mathrm{O}$. F. Müller, 1776

Recorded: Mangalia (Fleck, 1905 b; Negru \& Roşca, 1967); Periprava (Ieniştea, 1968); Maliuc, Babadag, Doloşman Cape (Razim Lake) (Crişan, 1993); Beştepe, Letea Forest, at the intersection of Sulina arm with Magearu Channel (Crişan, 1994); Lupilor sand bank (Sinoe Lake), Dunavăţ, Popina Island (Crişan, 1995 a); 
Dobrogea (without other data) (Skolka et al., 2005); Romanian Dobrogea (Gruev, 2005); Cetăţuia, Valea Seacă (Skolka et al., 2006-2007).

Material: Canaraua Fetii (Băneasa), 19.V.1993.

Distribution: panpalaearctic species, introduced also to North America.

Cassida sanguinolenta O. F. Müller, 1776

Recorded: Cernavodă (Fleck, 1905 b); Comarova Forest (Mangalia) (Negru, 1957; Negru \& Roşca, 1967).

Material: Dumbrăveni Forest, 18.V.1994.

Distribution: transpalaearctic species, distributed from Pyrenees and Ireland to Kamchatka.

\section{Cassida stigmatica Suffrian, 1844}

Recorded: Pricopan (Fleck, 1905 b).

Distribution: from Spain, W France and Belgium to N China.

Cassida subreticulata Suffrian, 1844

Recorded: Cernavodă (Fleck, 1905 b); Mangalia (Montandon, 1887, 1906; Negru \&

Roşca, 1967); Dobrogea (without other data) (Skolka et al., 2005).

Distribution: from Sicily, W Italy and Czech Republic to basin of Amur.

\section{Cassida vibex Linnaeus, 1767}

Recorded: Maliuc, Caraorman (Crişan, 1993); Agighiol (Crişan, 1994); Dobrogea (without other data) (Skolka et al., 2005).

Distribution: transpalaearctic species distributed from N Spain and Ireland to Japan. Cassida viridis Linnaeus, 1758

(syn: equestris Fabricius, 1787)

Recorded: Măcin Mountains, Isaccea (Montandon, 1887; 1906); Caraorman, Doloşman Cape (Razim Lake) (Crişan, 1993); at the intersection of Sulina arm with Magearu Channel (Crişan, 1994); Sfântu Gheorghe, Sacalin, Lupilor sand bank (Sinoe Lake), Dunavăţ (Crişan, 1995 a); Dobrogea (without other data) (Skolka et al., 2005); Măcin, Greci, Pricopan (Skolka et al., 2006-2007).

Distribution: transpalaearctic species distributed from the British Isles to Japan.

Hypocassida subferruginea (Schrank, 1776)

Recorded: Babadag, Constanţa (Montandon, 1887); Mangalia (Fleck, 1905 b; Montandon, 1908); Greci, Constanţa, Cernavodă, Babadag (Fleck, 1905 b; Montandon, 1908); Histria (Crişan, 1993); Beştepe (Crişan, 1994); Gura Portiţei, Sfântu-Gheorghe (Crişan, 1995 a); Dobrogea (without other data) (Skolka et al., 2005); Romanian Dobrogea (Gruev, 2005).

Material: 1 spec., Babadag, leg. Eduard Fleck; 1 spec., Mangalia, leg. Arnold Lucien Montandon.

Distribution: transpalaearctic species distributed from Morocco and the Great Britain to Korea.

\section{Pilemostoma fastuosum (Schaller, 1783)}

Recorded: Greci (Roşca, 1976; Skolka et al., 2006-2007); Letea Forest (Ieniştea, 1968); Romanian Dobrogea (Gruev, 2005).

Distribution: from the British Isles and France to the Baikal Lake.

\section{Tribe Hispini Gyllenhal, 1813}

Hispa atra Linnaeus, 1767

Recorded: Constanţa (Montandon, 1887; Fleck, 1905 b); Mangalia (Jaquet, 1901); Dobrogea (without other data) (Skolka et al., 2005).

Distribution: Europe, Mediterranean area, Near East, the Caucasus, W Asia to Mongolia and N China. 
Subfamily Chrysomelinae Latreille, 1802

Tribe Chrysomelini Latreille, 1802

Subtribe Chrysomelina Latreille, 1802

Chrysomela populi Linnaeus, 1758

Recorded: Comarova Forest (Mangalia), Iezeru (Negru, 1957; Negru \& Roşca, 1967); Mangalia (Roşca, 1974); Dobrogea (without other data) (Skolka et al., 2005); Romanian Dobrogea (Gruev, 2005).

Distribution: transpalaearctic species distributed from Ireland to Japan.

Chrysomela saliceti saliceti Weise, 1884

Recorded: Periprava (Negru, 1968 a).

Distribution: widely distributed from France to Mongolia.

Plagiodera versicolora (Laicharting, 1781)

Recorded: Măcin Mountains (Montandon, 1908; Roşca, 1976; Skolka et al., 20062007); at the intersection of Sulina arm with Magearu Channel (Crişan, 1994); Dunavăt, Popina Island (Razim Lake) (Crişan, 1995 a); Dobrogea (without other data) (Skolka et al., 2005); Romanian Dobrogea (Gruev, 2005).

Material: 5 specs, Măcin, leg. Arnold Lucien Montandon; Dunavăț, 9.VI.1994.

Distribution: Holarctic area.

Subtribe Gastrophysina Kippenberg, 2010

Gastrophysa (Gastrophysa) polygoni polygoni (Linnaeus, 1758)

Recorded: Constanţa, Babadag, Techirghiol Lake, Cernavodă, Murfatlar (Fleck, 1905 b); Comarova Forest (Mangalia), Hagieni Forest (Negru, 1957; Negru \& Roşca, 1967); Periprava (Negru, 1968 a); Babadag, Doloşman Cape (Razim Lake), Histria, Slava Rusă, Enisala (Crişan, 1993); Beştepe (Crişan, 1994); Gura Portiţei (Crişan, 1995 a); Agigea Natural Reserve (Serafim \& Maican, 2004); Dobrogea (without other data) (Skolka et al., 2005); Romanian Dobrogea (Gruev, 2005).

Distribution: all Europe, Asia Minor, the Caucasus, Central Asia.

Subtribe Prasocurina Reitteer, 1913

Neophaedon pyritosus (Rossi, 1792)

Recorded: Murfatlar (Fleck, 1905 b); Măcin (Montandon, 1906; Skolka et al., 20062007); Doloşman Cape (Razim Lake) (Crişan, 1993); Dobrogea (without other data) (Skolka et al., 2005); Romanian Dobrogea (Gruev, 2005); Greci, Pricopan (Skolka et al., 2006-2007).

Distribution: Central and SE Europe, Asia Minor, the Caucasus, Central Asia.

Phaedon (Phaedon) cochleariae cochleariae (Fabricius, 1792)

Recorded: at the intersection of Sulina arm with Magearu Channel (Crişan, 1994); Dobrogea (without other data) (Skolka et al., 2005).

Distribution: all Europe, Asia Minor, the Caucasus, Central Asia.

Phaedon (Phaedon) laevigatus laevigatus (Duftschmid, 1825)

(syn: sabulicola Suffrian, 1851)

Recorded: Pricopan crest, Orliga crest near Măcin (Jaquet, 1900 b); Turcoaia (Igliţa) Lake (Fleck, 1905 b); Romanian Dobrogea (Gruev, 2005); Măcin, Greci, Pricopan (Skolka et al., 2006-2007).

Distribution: S part of Central Europe, N Italy, Croatia, Bulgaria, Ukraine, the Caucasus.

Prasocuris (Prasocuris) phellandrii (Linnaeus, 1758)

Recorded: Doloşman Cape (Razim Lake) (Crişan, 1993); Dobrogea (without other data) (Skolka et al., 2005).

Distribution: Europe, N part of Asia Minor. 
Tribe Doryphorini Motschulsky, 1860

Subtribe Chrysolinina S.-H. Chen, 1936

Chrysolina (Chalcoidea) marginata marginata (Linnaeus, 1767)

Recorded: Babadag, Turcoaia (Iglița) Lake (Fleck, 1905 b); Măcin Mountains (Montandon, 1906, 1908; Skolka et al., 2006-2007); Măcin Mountains, Turcoaia (Igliţa) Lake (Roşca, 1976); Popina Island (Razim Lake) (Crişan, 1993); Canaraua Fetii (Băneasa) (Balog, 1998); Agigea (Serafim \& Maican, 2004); Dobrogea (without other data) (Skolka et al., 2005); Romanian Dobrogea (Gruev, 2005).

Material: 1 spec., Măcin, leg. Arnold Lucien Montandon.

Distribution: Europe, Central Asia from Caspian Sea to Sinkiang.

Chrysolina (Chrysolina) staphylaea staphylaea (Linnaeus, 1758)

Recorded: Olimp resort (North Mangalia) (Serafim \& Maican, 2004).

Distribution: from Ireland and Iceland to Kamchatka.

Chrysolina (Chrysomorpha) cerealis cerealis (Linnaeus, 1767)

Recorded: Enisala (Fleck, 1905 b); Măcin Mountains (Montandon, 1906; Skolka et al., 2006-2007); Greci, Pricopan (Skolka et al., 2006-2007).

Material: 1 spec., Măcin, leg. Arnold Lucien Montandon.

Distribution: transpalaearctic species distributed from Great Britain and N Spain to basins of Amur and Ussuri.

Chrysolina (Colaphodes) haemoptera haemoptera (Linnaeus, 1758)

Recorded: Eforie-Sud resort (Serafim \& Maican, 2004); Romanian Dobrogea (Gruev, 2005).

Distribution: from Portugal and S Great Britain to S part of Sweden and Finland, Asia Minor, the Caucasus, Central Asia, Himalaya.

Chrysolina (Colaphosoma) sturmi (Westhoff, 1882)

(syn: diversipes Bedel, 1892)

Recorded: Valu lui Traian (Roşca, 1974); Agighiol (Crişan, 1994); Canaraua Fetii (Băneasa) (Balog, 1998); Dobrogea (without other data) (Skolka et al., 2005); Romanian Dobrogea (Gruev, 2005). Material: Hagieni Forest, 22.V.1993, leg. Mihai Stănescu.

Distribution: from France and S-E Great Britain, Scandinavia, Central Europe, N Italy, basin of the Danube to Russia, Ukraine, the Caucasus.

\section{Chrysolina (Erythrochrysa) polita polita (Linnaeus, 1758)}

Recorded: Măcin Mountains, Isaccea (Montandon, 1887); Isaccea (Montandon, 1906; Roşca, 1976); Periprava (Negru, 1968 a); Caraorman sand bank (Ieniştea, 1974); Tulcea (Roşca, 1976); Maliuc, Enisala, Caraorman, Gorgova (Crişan, 1993); Sacalin, Sfântu Gheorghe, Dunavăț, Lupilor sand bank (Sinoe Lake) (Crişan, 1995 a); Dobrogea (without other data) (Skolka et al., 2005); Romanian Dobrogea (Gruev, 2005).

Material: Hagieni Forest, 22.V.1993.

Distribution: from N Spain, Ireland and S Norway to Mongolia and China.

Chrysolina (Euchrysolina) graminis graminis (Linnaeus, 1758)

Recorded: Mắcin Mountains (Montandon, 1906; Roşca, 1974, 1976; Skolka et al., 2006-2007); Tulcea (Roşca, 1976).

Material: 2 specs, Măcin, leg. Arnold Lucien Montandon.

Distribution: from Pyrenees, Great Britain, N Sweden to basins of the Black and Caspian Sea, Central Asia, Mongolia, N China.

Chrysolina (Fastuolina) fastuosa fastuosa (Scopoli, 1763)

Recorded: Enisala (Fleck, 1905 b); Hagieni Forest (Negru, 1957; Negru \& Roşca, 1967); Cocoş Monastery (Roşca, 1976); Slava Rusă, Babadag (Crişan, 1993); 
Agighiol (Crişan, 1994); Canaraua Fetii (Băneasa) (Balog, 1998); Dobrogea (without other data) (Skolka et al., 2005); Romanian Dobrogea (Gruev, 2005); Dobromir Forest (Maican, 2006 b); Valea Seacă, Greci (Skolka et al., 2006-2007).

Material: Negureni Forest, 18.V.1994, leg. Corneliu Pârvu.

Distribution: Europe, the Caucasus, Asia Minor.

Chrysolina (Hypericia) geminata (Paykull, 1799)

Recorded: Romanian Dobrogea (Gruev, 2005).

Distribution: Europe, from Pyrenees and Norway to Lithuania, Ukraine and Bulgaria.

Chrysolina (Hypericia) hyperici hyperici (Forster, 1771)

Recorded: Romanian Dobrogea (Gruev, 2005).

Distribution: Europa.

Chrysolina (Ovostoma) olivieri olivieri (Bedel, 1892)

Recorded: Romanian Dobrogea (Gruev, 2005).

Distribution: S Carpathians, Hungary, Romania (Transylvania), Serbia, S Slovakia, E Balkan Peninsula, Alps, Dinaric Alps, Stara Planina.

Chrysolina (Sphaeromela) varians (Schaller, 1783)

Recorded: Romanian Dobrogea (Gruev, 2005).

Distribution: Europe, W Siberia, introduced in North America.

Chrysolina (Stichoptera) gypsophilae (Küster, 1845)

Recorded: Cernavodă, Techirghiol Lake (Fleck, 1905 b); Periprava, C.A. Rosetti (Negru, 1968 a); Caraorman, Periprava (Roşca, 1974); Caraorman sand bank (Ieniştea, 1974); Letea Forest (Crișan, 1994); Canaraua Fetii (Băneasa) (Balog, 1998); Dobrogea (without other data) (Skolka et al., 2005); Romanian Dobrogea (Gruev, 2005); Măcin, Greci, Pricopan (Skolka et al., 2006-2007).

Distribution: NW Africa, Europe, Asia Minor, Central Asia.

Chrysolina (Stichoptera) kuesteri kuesteri (Helliesen, 1912)

Recorded: Romanian Dobrogea (Gruev, 2005).

Distribution: Iberian Peninsula, France, N Italy, Central Europe, basin of the Danube; Kiev.

\section{Chrysolina (Stichoptera) rossia (Illiger, 1802)}

Recorded: Periprava (Roșca, 1974); Agighiol (Crișan, 1994); Dobrogea (without other data) (Skolka et al., 2005); Romanian Dobrogea (Gruev, 2005).

Distribution: Italy, N part of Adriatic area.

Chrysolina (Stichoptera) sanguinolenta (Linnaeus, 1758)

Recorded: Măcin Mountains (Fleck, 1905 b; Montandon, 1906; Skolka et al., 20062007); Comarova Forest (Mangalia) (Negru \& Roşca, 1967); Valu lui Traian (Roşca, 1974); Popina Island (Razim Lake) (Crişan, 1993, 1995 a); Maliuc, Enisala (Crişan, 1993); Sfântu-Gheorghe (Crişan, 1995 a); Agigea (Serafim \& Maican, 2004); Dobrogea (without other data) (Skolka et al., 2005); Greci, Pricopan (Skolka et al., 2006-2007).

Material: 1 spec., Valu lui Traian, 18.X.1953, leg. Xenia Scobiola Palade.

Distribution: from Morocco, Great Britain and S part of Scandinavia to Mongolia.

Chrysolina (Sulcicollis) chalcites (Germar, 1824)

Recorded: Eforie-Sud resort (Panin, 1944); Enisala, Babadag (Crişan, 1993); Beştepe, Agighiol (Crişan, 1994); Gura Portiţei (Crişan, 1995 a); Agigea (Serafim \& Maican, 2004); Dobrogea (without other data) (Skolka et al., 2005); Romanian Dobrogea (Gruev, 2005); Dumbrăveni Forest (Maican, 2006 b).

Material: Canaraua Fetii (Băneasa), 20.V.1994.

Distribution: SE Europe, the Caucasus, Asia Minor, Near East, Central Asia. 
Chrysolina (Sulcicollis) oricalcia (O. F. Müller, 1776)

Recorded: Cocoş Monastery (Roşca, 1976); Romanian Dobrogea (Gruev, 2005).

Material: Dumbrăveni Forest, 18.V.1994.

Distribution: SE Great Britain, E France, Italy, Balkans, Central Europe, Ukraine, Crimea, Siberia.

Chrysolina (Synerga) coerulans coerulans (L. G. Scriba, 1791)

Recorded: Enisala (Fleck, 1905 b); Cocoş Monastery (Roşca, 1976); Romanian Dobrogea (Gruev, 2005).

Distribution: from Central France, Italy, Balkan to Asia Minor.

Chrysolina (Synerga) herbacea herbacea (Duftschmid, 1825)

Recorded: Măcin Mountains, Pricopan (Fleck, 1905 b; Roşca, 1974, 1976); Caraorman, Babadag, Slava Rusă (Crişan, 1993); Tuzla (Serafim \& Maican, 2004); Dobrogea (without other data) (Skolka et al., 2005); Romanian Dobrogea (Gruev, 2005); Măcin, Pricopan (Skolka et al., 2006-2007).

Material: 2 specs, Măcin, leg. Arnold Lucien Montandon.

Distribution: Europe, the Caucasus, Asia Minor, N Iran, Central Asia.

Chrysolina (Taeniosticha) reitteri (Weise, 1884)

Recorded: Măcin, Mangalia (Montandon, 1908).

Distribution: France, Central Europe, Bosnia, Crimea, Italy, basin of Danube, E Bulgaria, E Caucasus, Asia Minor.

Chrysolina (Zeugotaenia) limbata (Fabricius, 1775)

Recorded: Babadag, Mangalia (Montandon, 1887; Fleck, 1905 b); Dobrogea (without other data) (Skolka et al., 2005); Romanian Dobrogea (Gruev, 2005).

Distribution: from Pyrenees, W France and S Sweden to Siberia.

Oreina (Chrysochloa) cacaliae dinarica Apfelbeck, 1912

Recorded: Romanian Dobrogea (Gruev, 2005).

Distribution: Balkan Peninsula.

Subtribe Doryphorina Motschulsky, 1860

Leptinotarsa decemlineata (Say, 1824)

Recorded: Sulina (Negru, 1968 a); Cocoş Monastery (Roşca, 1976); Enisala (Crişan, 1993); Lupilor sand bank (Sinoe Lake) (Crişan, 1995 a); Dobrogea (without other data) (Skolka et al., 2005); Romanian Dobrogea (Gruev, 2005); Cetăţuia, Luncaviţa (Skolka et al., 2006-2007).

Distribution: introduced in Europe from North America.

Colaphellus sophiae sophiae (Schaller, 1783)

Recorded: Orliga (Măcin Mountains), Isaccea, Constanţa (Fleck, 1905 b); Comarova Forest (Mangalia), Hagieni Forest (Negru, 1957; Negru \& Roşca, 1967); Maliuc (Crişan, 1993); Beştepe, Agighiol, Iancina Cape (Crişan, 1994); Canaraua Fetii (Băneasa) (Balog, 1998); Agigea, Neptun resort (North Mangalia) (Serafim \& Maican, 2004); Dobrogea (without other data) (Skolka et al., 2005); Romanian Dobrogea (Gruev, 2005); Dumbrăveni Forest (Maican, 2006 b); Măcin, Greci, Pricopan (Skolka et al., 2006-2007).

Material: Canaraua Fetii (Băneasa), 19.V.1993, 20.V.1994; Hagieni Forest, 22.V.1993. Distribution: Central and SE Europe.

Entomoscelis adonidis (Pallas, 1771)

Recorded: Constanţa, Mangalia (Montandon, 1887; Fleck, 1905 b); Comarova Forest (Mangalia), Hagieni Forest, Băneasa (Negru, 1957; Negru \& Roşca, 1967); Periprava (Negru, 1968 a); Popina, Island (Razim Lake), Enisala (Crişan, 1993); Dobrogea (without other data) (Skolka et al., 2005); Romanian Dobrogea (Gruev, 2005).

Material: 1 spec., Valu lui Traian, 21.V.1955. 
Distribution: from S France to Mongolia.

Entomoscelis sacra (Linnaeus, 1758)

Recorded: Mangalia, Murfatlar, Cernavodă (Fleck, 1905 b); Măcin (Fleck, 1905 b; Skolka et al., 2006-2007); Greci (Montandon, 1906; Skolka et al., 2006-2007); Comarova Forest (Mangalia) (Negru, 1957; Negru \& Roşca, 1967); Dobrogea (without other data) (Skolka et al., 2005); Pricopan (Skolka et al., 2006-2007).

Material: 3 specs, Canaraua Fetii, 19.VI.1954, leg. Nicolae Săvulescu.

Distribution: Near East (according to Warchałowski, op. cit.).

Entomoscelis suturalis Weise, 1882

Recorded: Constanţa, Murfatlar (Fleck, 1905 b); Dobrogea (without other data) (Skolka et al., 2005); Lupilor sand bank (Sinoe Lake), Gura Portiţei (Crişan, 1995 a). Material: 2 specs, Babadag, leg. Eduard Fleck; 1 spec., Murfatlar, leg. Eduard Fleck; 2 specs, Constanța, leg. Arnold Lucien Montandon; 2 specs, Mangalia, leg. Arnold Lucien Montandon; Gura-Portiţei, 7.VI.1994, on Glaucium flavum.

Distribution: Romania, Bulgaria, S part of Ukraine, Caspian area, Asia Minor, the Caucasus, Near East.

Gonioctena (Gonioctena) linnaeana linnaeana (Schrank, 1781)

Recorded: Hagieni Forest (Negru \& Roşca, 1967); Dobrogea (without other data) (Skolka et al., 2005); Romanian Dobrogea (Gruev, 2005).

Distribution: from N Spain and Norway to Mongolia and Sakhalin.

Gonioctena (Spartomena) fornicata Brüggemann, 1873

Recorded: Mangalia (Montandon, 1908); Comarova Forest (Mangalia) (Negru \& Roşca, 1967); Dobrogea (without other data) (Skolka et al., 2005); Romanian Dobrogea (Gruev, 2005).

Distribution: basin of the Danube, S Poland, Balkan Peninsula, Ukraine, South Russia, the Caucasus, Asia Minor.

Tribe Timarchini Motschulsky, 1860

Timarcha (Timarcha) goettingensis goettingensis (Linnaeus, 1758)

(syn: violaceonigra, De Geer, 1775)

Recorded: Meledic, Mangalia (Fleck, 1905 b; Negru \& Roşca, 1967); Dobrogea (without other data) (Skolka et al., 2005).

Material: 2 specs, Mangalia, leg. Arnold Lucien Montandon.

Distribution: SW and Central Europe N to SW Poland and Romania.

Timarcha (Timarcha) pratensis (Duftschmid, 1825)

Recorded: Constanţa (Fleck, 1905 b); Hagieni Forest (Negru, 1957; Negru \& Roşca, 1967); Dobrogea (without other data) (Skolka et al., 2005); Romanian Dobrogea (Gruev, 2005).

Material: 1 spec., Mangalia, leg. Eduard Fleck.

Distribution: Austria, Bosnia and Herzegovina, Bulgaria, Croatia, Hungary, Italy, Montenegro, Romania, Slovenia (according to Löbl \& Smetana, 2010).

Subfamily Galerucinae Latreille, 1802

Tribe Galerucini Latreille, 1802

Galeruca (Galeruca) interrupta (Illiger, 1802)

Recorded: Orliga crest near Măcin (Jaquet, 1900 b); Hagieni Forest (Negru \& Roşca, 1967); Maliuc (Crişan, 1993); Canaraua Fetii (Băneasa) (Balog, 1998); Agigea (Serafim \& Maican, 2004); Dobrogea (without other data) (Skolka et al., 2005); Romanian Dobrogea (Gruev, 2005).

Distribution: W and N part of Europe. 
Galeruca (Galeruca) pomonae pomonae (Scopoli, 1763)

Recorded: Techirghiol Lake (Fleck, 1905 b); Hagieni Forest (Negru \& Roşca, 1967); Periprava (Negru, 1968 a); Caraorman (Ieniștea, 1974); Periteaşca (Crişan, 1995 a); Canaraua Fetii (Băneasa) (Balog, 1998); Dobrogea (without other data) (Skolka et al., 2005); Romanian Dobrogea (Gruev, 2005).

Distribution: from Portugal and Ireland to Central Asia; introduced in North America.

\section{Galeruca (Galeruca) tanaceti tanaceti (Linnaeus, 1758)}

Recorded: Tăbăcăria Lake (Constanţa) (Jaquet, 1898); Enisala (Fleck, 1905 b); Comarova Forest (Mangalia) (Negru, 1957; Negru \& Roșca, 1967); Periteaşca (Crişan, 1995 a); Canaraua Fetii (Băneasa) (Negru \& Roşca, 1967; Balog, 1998); Dobrogea (without other data) (Skolka et al., 2005); Romanian Dobrogea (Gruev, 2005).

Material: 2 specs, Valu lui Traian, 18.X.1953, leg. Xenia Scobiola Palade.

Distribution: from Ireland and Portugal to Korea; introduced in North America. Galeruca (Emarhopa) rufa Germar, 1824

Recorded: Techirghiol Lake (Fleck, 1905 b); Comarova Forest (Mangalia), Hagieni Forest (Negru, 1957; Negru \& Roşca, 1967); Canaraua Fetii (Băneasa) (Balog, 1998); Dobrogea (without other data) (Skolka et al., 2005); Romanian Dobrogea (Gruev, 2005).

Distribution: S France, Italy, basin of the Danube, Balkan Peninsula, Ukraine.

Galerucella (Galerucella) grisescens (Joannis, 1865)

Recorded: Sacalin (Crişan, 1995 a); Dobrogea (without other data) (Skolka et al., 2005).

Distribution: transpalaearctic species, distributed from Great Britain to Japan.

Galerucella (Galerucella) nymphaeae (Linnaeus, 1758)

Recorded: Comarova Forest (Mangalia) (Negru, 1957; Negru \& Roşca, 1967); Periprava, Sulina, C.A. Rosetti (Negru, 1968 a); Popina Island (Razim Lake), Caraorman, Doloşman Cape (Razim Lake) (Crişan, 1993); at the intersection of Sulina arm with Magearu Channel (Crişan, 1994); Sacalin (Crişan, 1995 a); Dobrogea (without other data) (Skolka et al., 2005); Romanian Dobrogea (Gruev, 2005).

Distribution: Europe.

$$
\text { Galerucella (Neogalerucella) calmariensis (Linnaeus, 1767) }
$$

Recorded: Chilia (the Danube Delta) (Ieniştea, 1968); Maliuc (Crişan, 1993); Dobrogea (without other data) (Skolka et al., 2005).

Distribution: transpalaearctic species, distributed from Catalonia and the British Isles to Japan.

\section{Galerucella (Neogalerucella) lineola lineola (Fabricius, 1781)}

Recorded: Cocoș Monastery (Montandon, 1887); Tulcea (Montandon, 1906); Greci (Montandon, 1908); at the intersection of Sulina arm with Magearu Channel (Crişan, 1994); Dunavăţ, Sacalin (Crişan, 1995 a); Dobrogea (without other data) (Skolka et al., 2005); Romanian Dobrogea (Gruev, 2005).

Distribution: tranpalaearctic species, distributed from Ireland to Japan.

Galerucella (Neogalerucella) pusilla (Duftschmid, 1825)

Recorded: Greci (Montandon, 1908); Doloşman Cape (Razim Lake) (Crişan, 1993); Dunavăț (Crişan, 1995 a); Dobrogea (without other data) (Skolka et al., 2005); Romanian Dobrogea (Gruev, 2005).

Distribution: from Catalonia and the British Isles to Mongolia. Lochmaea crataegi (Forster, 1771)

Recorded: Cocoş Monastery (Montandon, 1906). 
Distribution: from N Spain and Great Britain to Asia Minor, the Caucasus, basin of Volga and Mongolia.

Xanthogaleruca luteola (O. F. Müller, 1766)

(syn: xanthomelaena Schrank, 1781)

Recorded: Cocoş Monastery (Montandon, 1906); Greci (Montandon, 1908); Comarova Forest (Mangalia) (Negru, 1957; Negru \& Roşca, 1967); Dobrogea (without other data) (Skolka et al., 2005); Romanian Dobrogea (Gruev, 2005); Măcin, Greci, Pricopan (Skolka et al., 2006-2007).

Distribution: from Portugal, W France and Denmark to the Caucasus and Central Asia.

Tribe Hylaspini Chapuis, 1875

Agelastica alni alni (Linnaeus, 1758)

Recorded: Romanian Dobrogea (Gruev, 2005).

Distribution: Europe, from Ireland to S Sweden and from Pyrenees to Asia Minor and Caucasian countries.

Tribe Luperini Gistel, 1848

Subtribe Luperina Gistel, 1848

Euluperus cyaneus (Joannis, 1865)

Material: 3 specs, Babadag, leg. Eduard Fleck.

Distribution: SW part of Balkan Peninsula.

Luperus xanthopoda Schrank, 1781

Recorded: Romanian Dobrogea (Gruev, 2005).

Distribution: from Iberian Peninsula to Central Asia.

Phyllobrotica adusta adusta (Creutzer, 1799)

Recorded: Babadag (Fleck, 1905 b); Hagieni Forest (Negru \& Roşca, 1967); Greci (Roşca, 1976); Olimp resort (North Mangalia) (Serafim \& Maican, 2004); Dobrogea (without other data) (Vasiliu-Oromulu et al., 2004; Skolka et al., 2005); Greci (Skolka et al., 2006-2007).

Distribution: Balkan Peninsula, basin of the Danube and Ukraine.

Phyllobrotica quadrimaculata (Linnaeus, 1758)

Recorded: Caraorman sand bank (Ieniştea, 1974); Dunavăţ (Crişan , 1995 a); Dobrogea (without other data) (Skolka et al., 2005).

Distribution: Europe, from Ireland and S Fennoscandia to basin of Ural and from Pyrenees and N Italy to Bulgaria.

Subfamily Alticinae Newman, 1835

Altica brevicollis brevicollis Foudras, 1861

Recorded: Romanian Dobrogea (Gruev, 2005).

Distribution: Europe, from N Spain and Great Britain to basin of Volga, in Asia Minor, the Caucasian countries, Iran and Kazakhstan.

Altica carduorum Guérin-Méneville, 1858

Recorded: Histria, Enisala, Gorgova, Maliuc, Slava Rusă (Crişan, 1993); Beştepe, Gorgova (Crişan, 1994); Sfântu Gheorghe, Dunavăţ (Crişan, 1995 a); Dobrogea (without other data) (Gruev, 2001; Skolka et al., 2005); Romanian Dobrogea (Gruev, 2005).

Material: Hagieni Forest, 22.V.1993.

Distribution: central and S part of Europe, Asia Minor, the Caucasus, Central Asia. 
Altica fruticola (Weise, 1888)

Recorded: Greci (Montandon, 1908); Dobrogea (without other data) (Gruev, 2001); Romanian Dobrogea (Gruev, 2005); Măcin, Greci, Pricopan (Skolka et al., 20062007).

Distribution: reported from different countries (Spain, Austria, Ukraine).

Altica helianthemi (Allard, 1859)

(syn: pusilla Duftschmid, 1825)

Recorded: Pricopan Crest near Măcin (Jaquet, 1900 a; Fleck, 1905 b); Dobrogea (without other data) (Gruev, 2001); Romanian Dobrogea (Gruev, 2005).

Distribution: Europe, Asia.

Altica impressicollis (Reiche, 1862)

Recorded: Dunavăț (Crişan, 1995 a); Dobrogea (without other data) (Skolka et al., 2005).

Distribution: S and central part of Europe, Asia Minor, Near East, the Caucasus, N Iran.

Altica lythri Aubé, 1843

Recorded: Dobrogea (without other data) (Gruev, 2001); Romanian Dobrogea (Gruev, 2005).

Distribution: Europe, Turkey.

Altica oleracea oleracea (Linnaeus, 1758)

Recorded: Pricopan Crest near Măcin (Jaquet, 1900 a); Cernavodă (Hurmuzachi, 1904; Fleck, 1905 b); Babadag, Tulcea, Orliga (Fleck, 1905 b; Ionescu-Konnerth, 1963); Hagieni Forest (Negru \& Roşca, 1967); Popina Island (Razim Lake), Maliuc, Babadag, Doloşman Cape (Razim Lake), Enisala, Caraorman, Histria (Crişan, 1993); Dunavăț (Crişan, 1995 a); Agigea Natural Reserve (Serafim \& Maican, 2004); Dobrogea (without other data) (Gruev, 2001; Vasiliu-Oromulu et al., 2004; Skolka et al., 2005); Romanian Dobrogea (Gruev, 2005); Măcin, Greci, Pricopan (Skolka et al., 2006-2007).

Distribution: transpalaearctic species, distributed from Spain and Ireland to Japan. Altica quercetorum quercetorum Foudras, 1861

Material: Negureni Forest, 17.V.1993, leg. Mihaela Barcan.

Distribution: Europe, from N Spain, Netherlands and S Norway to basin of Volga, Asia Minor, the Caucasus.

\section{Altica tamaricis tamaricis Schrank, 1785}

Recorded: Popina Island (Razim Lake), Doloşman Cape (Razim Lake), Caraorman, Babadag, Gorgova, Slava Rusă (Crişan, 1993); Sfântu-Gheorghe, Dunavăţ (Crişan, 1995 a); Dobrogea (without other data) (Skolka et al., 2005).

Distribution: Europe, Asia Minor, Near East, the Caucasus, Iran, Central Asia, Mongolia, Siberia.

\section{Aphtona abdominalis (Duftschmid, 1825)}

Recorded: Greci (Montandon, 1908); Beştepe, Agighiol (Crişan, 1994); Dobrogea (without other data) (Gruev, 2001; Skolka et al., 2005); Romanian Dobrogea (Gruev, 2005); Măcin, Greci, Pricopan (Skolka et al., 2006-2007).

Distribution: Europe, W Asia, from France to Kazakhstan.

\section{Aphtona atrocaerulea (Stephens, 1831)}

(syn: cyanella L. Redtenbacher, 1849)

Recorded: Sfântu Gheorghe (Crişan, 1995 a); Dobrogea (without other data) (Skolka et al., 2005).

Distribution: W and Central Europe, N Africa. 
Aphtona cyparissiae (Koch, 1803)

Recorded: Mangalia, Constanţa (Jaquet, 1900 a; Fleck, 1905 b; Ionescu-Konnerth, 1963; Negru \& Roşca, 1967); Constanţa, Techirghiol Lake (Fleck, 1905 b; IonescuKonnerth, 1963); Maliuc (Crişan, 1993); Beştepe, Agighiol, Iancina Cape (Crişan, 1994); Comarova Forest (Mangalia), Agigea (Nagy, 1972; Gomoiu \& Skolka, 1998); Dobrogea (without other data) (Gruev, 2001; Skolka et al., 2005); Romanian Dobrogea (Gruev, 2005).

\section{Aphtona euphorbiae (Schrank, 1781)}

Recorded: Popina Island (Razim Lake), Caraorman, Slava Rusă (Crişan, 1993); Beştepe (Crişan, 1994); Lupilor sand bank (Sinoe Lake), Periteaşca, Gura Portiței, Dunavăț (Crişan, 1995 a); Dobrogea (without other data) (Gruev, 2001; Skolka et al., 2005); Romanian Dobrogea (Gruev, 2005).

Distribution: W Palaearctic species, distributed from Morocco, Portugal and Ireland to W Siberia, Near East, Asia Minor and the Caucasus.

\section{Aphtona franzi Heikertinger, 1944}

Recorded: Dobrogea (without other data) (Gruev, 2001); Romanian Dobrogea (Gruev, 2005).

Distribution: the Danube basin, Romania, Bulgaria, Asia Minor, N Syria, S Ukraine, S Russia.

Aphtona gracilis Faldermann, 1837

Recorded: Romanian Dobrogea (Gruev, 2005).

Distribution: from Romania, Asia Minor and Israel to Central Asia and Transbaicalia. Aphtona lutescens (Gyllenhal, 1813)

Recorded: Sfântu Gheorghe, Dunavăţ (Crişan, 1995 a); Dobrogea (without other data) (Gruev, 2001; Skolka et al., 2005); Caraorman (Gruev, 2002); Romanian Dobrogea (Gruev, 2005).

Distribution: Europe, Asia Minor, the Caucasus, Central Asia, Mongolia. Aphtona nigriscutis Foudras, 1860

Recorded: Beştepe, Letea Forest, Iancina Cape, Agighiol (Crişan, 1994); Sfântu Gheorghe, Lupilor sand bank (Sinoe Lake), Periteaşca (Crişan, 1995 a); Dobrogea (without other data) (Gruev, 2001; Skolka et al., 2005); Romanian Dobrogea (Gruev, 2005).

Distribution: SE Europe from N Italy, E Austria, Slovakia and central Poland to Crete, Cyprus, Israel, the Caucasus.

Aphtona nonstriata (Goeze, 1777)

(syn: coerulea Geoffroy, 1785)

Recorded: Dunavăţ, Sfântu-Gheorghe (Crişan, 1995 a); Dobrogea (without other data) (Skolka et al., 2005).

Distribution: Europe from Ireland and Pyrenees to Turkey, the Caucasus, Iran, N Kazakhstan, Canary Islands. Aphtona placida (Kutschera, 1864)

Recorded: Doloşman Cape (Razim Lake) (Crişan, 1993); Dobrogea (without other data) (Skolka et al., 2005).

Distribution: Danube basin, Istria, S Poland. Aphtona pygmaea (Kutschera, 1861)

Recorded: Dobrogea (without other data) (Gruev, 2001); Romanian Dobrogea (Gruev, 2005).

Distribution: E France, S and central parts of Germany and Poland, Ukraine, the Caucasus, Italy, Austria, the Danube basin, Balkan Peninsula, Asia Minor, Near East, Egypt, Syria. 
Aphtona venustula (Kutschera, 1861)

Recorded: Gorgova (Crişan, 1994); Dobrogea (without other data) (Gruev, 2001; Skolka et al., 2005); Romanian Dobrogea (Gruev, 2005).

Distribution: from Pyrenees and Ireland to Ukraine, Asia Minor, the Caucasus. Aphtona violacea (Koch, 1803)

Recorded: Lupilor sand bank (Sinoe Lake) (Crişan, 1995 a); Dobrogea (without other data) (Gruev, 2001; Skolka et al., 2005); Şontea channel, Caraorman (Gruev, 2002); Romanian Dobrogea (Gruev, 2005).

Distribution: Europe, S Siberia, the Caucasus. Argopus ahrensii (Germar, 1817)

Recorded: Babadag (Crişan, 1993); Dobrogea (without other data) (Skolka et al., 2005).

Distribution: Europe from Italy and Austria to Greece, Romania, SE Poland, Ukraine, Central Russia.

Chaetocnema (Chaetocnema) aerosa (Letzner, 1847)

Recorded: Sfântu Gheorghe (Crişan, 1995 a); Dobrogea (without other data) (Skolka et al., 2005).

Distribution: Europe, the Caucasus, Asia Minor.

\section{Chaetocnema (Chaetocnema) aridula (Gyllenhal, 1827)}

Recorded: Constanţa (Montandon, 1887); Mangalia (Montandon, 1887; Fleck, 1905 b); Babadag, Constanța (Fleck, 1905 b; Ionescu-Konnerth, 1963; Negru \& Roşca, 1967); Dobrogea (without other data) (Skolka et al., 2005); Romanian Dobrogea (Gruev, 2005).

Distribution: Europe, Mediterranean area, Asia Minor, the Caucasus, Central Asia, Siberia.

Chaetocnema (Chaetocnema) compressa (Letzner, 1847)

Recorded: Dobrogea (without other data) (Gruev, 2001); Romanian Dobrogea (Gruev, 2005).

Distribution: Central and E part of Europe; reported from Switzerland, the Caucasus, Kazakhstan.

Chaetocnema (Chaetocnema) hortensis (Geoffroy, 1785)

Recorded: Măcin Mountains, Greci (Montandon, 1908); Dobrogea (without other data) (Gruev, 2001); Romanian Dobrogea (Gruev, 2005); Măcin, Greci, Pricopan (Skolka et al., 2006-2007).

Distribution: Palaearctic species, distributed from Azores and Great Britain to Far East.

Chaetocnema (Chaetocnema) obesa (Boieldieu, 1859)

Recorded: Dobrogea (without other data) (Gruev, 2001); Romanian Dobrogea (Gruev, 2005).

Distribution: from Algeria, N Spain and France to Mongolia and Tibet.

Chaetocnema (Tlanoma) breviuscula (Faldermann, 1837)

Recorded: Dobrogea (without other data) (Gruev, 2001, 2005).

Distribution: from E Europe (Balkans, Hungary) to Korea.

Chaetocnema (Tlanoma) chlorophana (Duftschmid, 1825)

Recorded: Constanţa (Montandon, 1887; Fleck, 1905 b); Dobrogea (without other data) (Gruev, 2001); Dobrogea (without other data) (Skolka et al., 2005); Romanian Dobrogea (Gruev, 2005).

Distribution: Mediterranean area, S Europe, northwards to Belgium, basin of Danube and Ukraine, Asia Minor, the Caucasus, Near East. 
Chaetocnema (Tlanoma) concinna (Marsham, 1802)

Recorded: Greci (Montandon, 1908); at the intersection of Sulina arm with Magearu Channel (Crişan, 1994); Lupilor sand bank (Sinoe Lake), Dunavăţ (Crişan, 1995 a); Dobrogea (without other data) (Gruev, 2001; Skolka et al., 2005); Romanian Dobrogea (Gruev, 2005); Măcin, Greci, Pricopan (Skolka et al., 2006-2007).

Distribution: Panpalaearctic species, introduced also to Canada.

Chaetocnema (Tlanoma) conducta (Motschulsky, 1838)

Recorded: Pricopan Crest, near Măcin, Orliga (Jaquet, 1900); Lupilor sand bank (Sinoe Lake) (Crişan, 1995 a); Dobrogea (without other data) (Gruev, 2001; Skolka et al., 2005); Romanian Dobrogea (Gruev, 2005).

Distribution: S part of Europe, Africa, Asia Minor, the Caucasus, Near East, Central Asia.

Chaetocnema (Tlanoma) major (Jacquelin du Val, 1852)

Recorded: Constanţa (Montandon, 1887; Fleck, 1905 b); Dobrogea (without other data) (Gruev, 2001; Skolka et al., 2005); Romanian Dobrogea (Gruev, 2005).

Distribution: S part of Europe, Asia Minor, the Caucasus, Near East, Iran, Kazakhstan, China, Japan.

\section{Chaetocnema (Tlanoma) tibialis (Illiger, 1807)}

Recorded: Constanţa (Montandon, 1887; Hurmuzachi, 1904); Măcin, Turcoaia (Igliţa) Lake (Montandon, 1908; Roşca, 1976); Constanţa, Măcin (Ionescu-Konnerth, 1963); Slava Rusă (Crişan, 1993); Enisala: Cetatea Heraclea (Vig, 1997); Dobrogea (without other data) (Gruev, 2001; Skolka et al., 2005); Romanian Dobrogea (Gruev, 2005); Măcin (Skolka et al., 2006-2007).

Material: 16 specs, Măcin, leg. Arnold Lucien Montandon.

Distribution: Europe, N Africa, Asia Minor, the Caucasus, Syria, Central Asia, W Siberia, Mongolia.

Crepidodera aurata (Marsham, 1802)

Recorded: Babadag (Fleck, 1905 b); Maliuc (Crişan, 1993); Gorgova, at the intersection of Sulina arm with Magearu Channel (Crişan, 1994); Dunavăţ (Crişan, 1995 a); Dobrogea (without other data) (Gruev, 2001; Skolka et al., 2005); Romanian Dobrogea (Gruev, 2005).

Material: Hagieni Forest, 22.V.1993.

Distribution: Europe and Asia from Spain and Ireland to Siberia, reported also from Morocco.

\section{Crepidodera fulvicornis (Fabricius, 1792)}

Recorded: Mangalia (Jaquet, 1900 a; Fleck, 1905 b; Negru \& Roşca, 1967; Nagy, 1972); Dobrogea (without other data) (Gruev, 2001; Skolka et al., 2005); Romanian Dobrogea (Gruev, 2005).

Distribution: from Spain and the British Isles to Mongolia and basin of Amur.

Crepidodera plutus (Latreille, 1804)

(syn: chloris Foudras, 1861)

Recorded: Greci (Montandon, 1908); Măcin, Tulcea, Niculițel (Roşca, 1976); Popina Island (Razim Lake), Gorgova, Maliuc (Crişan, 1993); Letea Forest, Gorgova, at the intersection of Sulina arm with Magearu Channel (Crişan, 1994); Dunavăt, Sfântu Gheorghe (Crişan, 1995 a); Dobrogea (without other data) (Gruev, 2001; Skolka et al., 2005); Romanian Dobrogea (Gruev, 2005); Măcin, Greci, Pricopan (Skolka et al., 2006-2007).

Material: Canaraua Fetii (Băneasa), 20.V.1994.

Distribution: transpalaearctic species, distributed from Spain and the British Isles to Japan. 
Dibolia (Dibolia) cryptocephala (Koch, 1803)

Recorded: Greci (Montandon, 1908); Babadag, Gorgova (Crişan, 1993); Dobrogea (without other data) (Gruev, 2001; Skolka et al., 2005); Romanian Dobrogea (Gruev, 2005); Măcin, Greci, Pricopan (Skolka et al., 2006-2007).

Distribution: Europe from Pyrenees to S Ural Mountains.

\section{Dibolia (Dibolia) foersteri Bach, 1859}

Recorded: Dobrogea (without other data) (Gruev, 2001); Romanian Dobrogea (Gruev, 2005).

Distribution: Europe from Spain and Belgium to Ukraine, reported from Armenia. Dibolia (Dibolia) occultans (Koch, 1803)

Recorded: Pricopan crest near Măcin (Jaquet, 1900 b).

Distribution: Europe except the British Isles; reported also from Caucasian countries, Canary Islands, Morocco, Algeria.

Dibolia (Eudibolia) schillingii (Letzner, 1847)

Recorded: Mangalia (Jaquet, 1900 a; Fleck, 1905 b; Montandon, 1908; IonescuKonnerth, 1963; Negru \& Roşca, 1967); Măcin Mountains, Greci (Montandon, 1908); Greci (Fleck, 1905 b; Ionescu-Konnerth, 1963); Dobrogea (without other data) (Skolka et al., 2005); Romanian Dobrogea (Gruev, 2005).

Distribution: from N Italy and Belgium to S Russia, Turkey, the Caucasus.

Epitrix pubescens (Koch, 1803)

Recorded: Greci (Montandon, 1908); Dobrogea (without other data) (Gruev, 2001); Tulcea (Gruev, 2002); Dobrogea (without other data) (Skolka et al., 2005); Romanian Dobrogea (Gruev, 2005).

Distribution: from Azores to W Siberia.

Longitarsus (Longitarsus) albineus (Foudras, 1860)

Recorded: Dobrogea (without other data) (Gruev, 2001); Romanian Dobrogea (Gruev, 2005).

Distribution: Mediterranean area, Asia Minor, Central Asia.

\section{Longitarsus (Longitarsus) apicalis (Beck, 1817)}

Recorded: Maliuc (Crişan, 1993); Dobrogea (without other data) (Skolka et al., 2005). Distribution: N and Central Europe.

\section{Longitarsus (Longitarsus) ferrugineus (Foudras, 1860)}

(syn: waterhousei Kutschera, 1864)

Recorded: at the intersection of Sulina arm with Magearu Channel (Crişan, 1994); Dobrogea (without other data) (Skolka et al., 2005).

Distribution: NW Africa, Europe from central Spain and Great Britain to Italy, Hungary, S Sweden, W Russia.

\section{Longitarsus (Longitarsus) foudrasi Weise, 1893}

Recorded: Lupilor sand bank (Sinoe Lake) (Crişan, 1995 a).

Distribution: SE part of Europe, Asia Minor; reported also from Spain, Madeira and Morocco.

\section{Longitarsus (Longitarsus) juncicola (Foudras, 1860)}

Recorded: Dobrogea (without other data) (Gruev, 2001); Romanian Dobrogea (Gruev, 2005).

Distribution: Mediterranean part of Europe.

Longitarsus (Longitarsus) linnaei (Duftschmid, 1825)

Recorded: Valea Fagilor Forest (Skolka et al., 2006-2007).

Distribution: Europe, the Caucasus, Asia Minor, Central Asia. 
Longitarsus (Longitarsus) luridus luridus (Scopoli, 1763)

Recorded: Dobrogea (without other data) (Gruev, 2001); Romanian Dobrogea (Gruev, 2005).

Distribution: from Morocco and Ireland to Kamchatka and Ussuri.

Longitarsus (Longitarsus) lycopi (Foudras, 1860)

Recorded: Lupilor sand bank (Sinoe Lake), Periteaşca (Crişan, 1995 a); Dobrogea (without other data) (Gruev, 2001; Skolka et al., 2005); Romanian Dobrogea (Gruev, 2005).

Distribution: W Palaearctic.

Longitarsus (Longitarsus) melanocephalus (De Geer, 1775)

Recorded: Dobrogea (without other data) (Gruev, 2001); Romanian Dobrogea (Gruev, 2005).

Distribution: from Ireland and W coasts of Europe to Mongolia.

Longitarsus (Longitarsus) nanus (Foudras, 1860)

Recorded: Enisala, Babadag (Crişan, 1993); Dobrogea (without other data) (Skolka et al., 2005).

Distribution: S Europe.

Longitarsus (Longitarsus) nigrofasciatus nigrofasciatus (Goeze, 1777)

Recorded: Măcin Mountains, Pricopan (Ionescu-Konnerth, 1963; Skolka et al., 2006-2007); Dunavăț (Crişan, 1995 a); Dobrogea (without other data) (Gruev, 2001; Skolka et al., 2005); Romanian Dobrogea (Gruev, 2005).

Distribution: W Palaearctic.

$$
\text { Longitarsus (Longitarsus) obliteratus (Rosenhauer, 1847) }
$$

Recorded: Greci (Montandon, 1908); Dobrogea (without other data) (Gruev, 2001); Romanian Dobrogea (Gruev, 2005); Măcin, Greci, Pricopan (Skolka et al., 20062007).

Distribution: Europe, NW Africa.

$$
\text { Longitarsus (Longitarsus) parvulus (Paykull, 1799) }
$$

Recorded: Dobrogea (without other data) (Gruev, 2001); Romanian Dobrogea (Gruev, 2005).

Distribution: from Cape Verde Islands and Madeira to E Siberia.

$$
\text { Longitarsus (Longitarsus) pellucidus (Foudras, 1860) }
$$

Recorded: Constanţa (Fleck, 1905 b); Dobrogea (without other data) (Gruev, 2001; Skolka et al., 2005); Romanian Dobrogea (Gruev, 2005).

Distribution: W Palaearctic species, distributed from Madeira, Morocco, Iberian Peninsula, the British Isles to Mongolia.

\section{Longitarsus (Longitarsus) picicollis Weise, 1900}

Recorded: Dobrogea (without other data) (Gruev, 2001); Romanian Dobrogea (Gruev, 2005).

Distribution: E part of Balkan Peninsula, Crimea, Asia Minor, the Caucasus, Iran, Central Asia.

\section{Longitarsus (Longitarsus) pratensis (Panzer, 1794)}

Recorded: Dobrogea (without other data) (Gruev, 2001); Romanian Dobrogea (Gruev, 2005).

Distribution: Europe, Asia Minor, Central Asia.

Longitarsus (Longitarsus) reichei (Allard, 1860)

Recorded: Dobrogea (without other data) (Gruev, 2001); Romanian Dobrogea (Gruev, 2005).

Distribution: W and Central Europe. 
Longitarsus (Longitarsus) substriatus Kutshera, 1864

Recorded: Iancina Cape, at the intersection of Sulina arm with Magearu Channel (Crişan, 1994); Dobrogea (without other data) (Gruev, 2001; Skolka et al., 2005); Romanian Dobrogea (Gruev, 2005).

Distribution: Italy, Austria, Slovakia, Balkan Peninsula, reported also from France.

Longitarsus (Longitarsus) succineus (Foudras, 1860)

Recorded: Dobrogea (without other data) (Gruev, 2001); Romanian Dobrogea (Gruev, 2005).

Distribution: Europe, Asia, from Iberian Peninsula and the British Isles to Japan. Longitarsus (Longitarsus) suturellus (Duftschmid, 1825)

Recorded: Caraorman (Gruev, 2002); Romanian Dobrogea (Gruev, 2005).

Distribution: transpalaearctic species, distributed from Ireland to Japan. Longitarsus (Longitarsus) tabidus tabidus (Fabricius, 1775)

(syn: verbasci Panzer, 1794)

Recorded: Pricopan (Jaquet, 1900 a); Babadag (Fleck, 1905 b; Babadag, Comarova Forest (Mangalia) (Negru, 1957; Ionescu-Konnerth, 1963; Negru \& Roşca, 1967); Greci (Montandon, 1908); Dunavăț (Crișan, 1995 a); Dobrogea (without other data) (Gruev, 2001; Skolka et al., 2005); Romanian Dobrogea (Gruev, 2005); Măcin, Greci, Pricopan (Skolka et al., 2006-2007).

Distribution: W part of Palaearctic region, from Morocco to Mongolia.

Longitarsus (Testergus) anchusae (Paykull, 1799)

Recorded: Gura Portiţei, Periteaşca (Crişan, 1995 a); Dobrogea (without other data) (Skolka et al., 2005); Romanian Dobrogea (Gruev, 2005).

Distribution: from Pyrenees and Ireland to Afghanistan, in SE Europe, Asia Minor, Israel, the Caucasus.

\section{Lythraria salicariae (Paykull, 1800)}

Recorded: Caraorman (Gruev, 2002); Romanian Dobrogea (Gruev, 2005).

Distribution: from Pyrenees, Great Britain and Norway to Korea.

Neocrepidodera ferruginea (Scopoli, 1763)

Recorded: Mangalia (Montandon, 1908; Ionescu-Konnerth, 1963; Negru \& Roşca, 1967), Greci (Montandon, 1908; Ionescu-Konnerth, 1963; Gruev, 2001); Letea Forest (Crişan, 1994); Dobrogea (without other data) (Skolka et al., 2005); Romanian Dobrogea (Gruev, 2005); Măcin, Greci, Pricopan (Skolka et al., 2006-2007).

Distribution: Europe, Asia Minor, the Caucasus, Iran, Azores.

Neocrepidodera transversa (Marsham, 1802)

Recorded: Histria, Gorgova, Babadag (Crişan, 1993); at the intersection of Sulina arm with Magearu Channel (Crişan, 1994); Sfântu Gheorghe, Periteaşca, Gura Portiţei, Lupilor sand bank (Sinoe Lake) (Crişan, 1995 a); Mamaia (Serafim \& Maican, 2004); Dobrogea (without other data) (Gruev, 2001; Skolka et al., 2005). Material: Gura-Portiței, 3.VI.1994.

Distribution: Europe, from Portugal and Ireland to Caspian Sea, Asia Minor, Near East Caucasus, Iran, Afghanistan.

\section{Phyllotreta atra (Fabricius, 1775)}

Recorded: Constanţa (Montandon, 1887); Mangalia, Constanţa, Valu lui Traian (Fleck, 1905 b; Ionescu-Konnerth, 1963; Negru \& Roşca, 1967); Dobrogea (Gruev, 2001; Skolka et al., 2005); Romanian Dobrogea (Gruev, 2005).

Distribution: Europe, W part of Asia to Mongolia; reported from Morocco.

Phyllotreta balcanica Heikertinger, 1909

Recorded: Dobrogea (without other data) (Gruev, 2001); Romanian Dobrogea (Gruev, 2005). 
Distribution: S and SE part of Europe from France to Hungary, Greece, S Russia, Asia Minor, the Caucasus.

\section{Phyllotreta cruciferae (Goeze, 1777)}

Recorded: Măcin (Fleck, 1905 b); Greci (Montandon, 1908); Mangalia, Constanţa (Fleck, 1905 b; Ionescu-Konnerth, 1963; Negru \& Roşca, 1967); Comarova Forest (Mangalia) (Nagy, 1972; Gomoiu \& Skolka, 1998); Dobrogea (without other data) (Gruev, 2001; Skolka et al., 2005); Romanian Dobrogea (Gruev, 2005).

Distribution: W part of Palaearctic region from Morocco to Central Asia, India, Sudan; introduced also in North America.

\section{Phyllotreta diademata Foudras, 1860}

Recorded: Greci (Montandon, 1887); Mangalia (Fleck, 1905 b; Ionescu-Konnerth, 1963; Negru \& Roșca, 1967); Măcin Mountains (Fleck, 1905 b; Ionescu-Konnerth, 1963); Dobrogea (without other data) (Gruev, 2001; Skolka et al., 2005); Romanian Dobrogea (Gruev, 2005); Măcin, Greci, Pricopan (Skolka et al., 2006-2007).

Distribution: Europe, Asia Minor, Central Asia; reported from India.

Phyllotreta dilatata C. G. Thomson, 1866

Recorded: Gura Portiţei, Lupilor sand bank (Sinoe Lake) (Crişan, 1995 a); Dobrogea (without other data) (Skolka et al., 2005).

Distribution: W and central part of Europe, Ukraine and Asiatic Russia.

Phyllotreta nemorum (Linnaeus, 1758)

Recorded: Comarova Forest (Mangalia), Hagieni Forest (Ionescu-Konnerth, 1963; Negru, 1957; Negru \& Roşca, 1967); Valu lui Traian, Constanţa, Comarova Forest (Mangalia), Hagieni Forest (Ionescu-Konnerth, 1963); Dobrogea (without other data) (Gruev, 2001; Skolka et al., 2005); Romanian Dobrogea (Gruev, 2005); Măcin, Greci, Pricopan (Skolka et al., 2006-2007).

Distribution: transpalaearctic species, distributed from Ireland and Spain to Korea; introduced to Australia.

\section{Phyllotreta nigripes nigripes (Fabricius, 1775)}

Recorded: Greci (Montandon, 1908); Valu lui Traian (Ionescu-Konnerth, 1963, with older data); Dobrogea (without other data) (Gruev, 2001; Skolka et al., 2005); Romanian Dobrogea (Gruev, 2005); Măcin, Greci, Pricopan (Skolka et al., 20062007).

Distribution: Mediterranean area, Europe, Asia Minor, the Caucasus, Central Asia. Phyllotreta ochripes (Curtis, 1837)

Recorded: Constanța (Fleck, 1905 b; Montandon, 1887); Dobrogea (Gruev, 2001; Skolka et al., 2005); Romanian Dobrogea (Gruev, 2005); Valea Fagilor Forest (Skolka et al., 2006-2007).

Distribution: Europe, Asia Minor, the Caucasus, Iran and Siberia to Russian Far East.

\section{Phyllotreta procera (L. Redtenbacher, 1849)}

Recorded: Greci (Montandon, 1908; Ionescu-Konnerth, 1963); Dobrogea (without other data) (Gruev, 2001; Skolka et al., 2005); Romanian Dobrogea (Gruev, 2005); Măcin, Greci, Pricopan (Skolka et al., 2006-2007).

Distribution: Mediterranean area, S part of Central Europe, Balkan Peninsula, Asia Minor, Near East.

Phyllotreta punctulata (Marsham, 1802)

Recorded: Dobrogea (without other data) (Gruev, 2001; 2005).

Distribution: S and central part of Europe, Ukraine, Morocco, Asia Minor, Israel, the Caucasus; introduced to USA. 
Phyllotreta striolata (Fabricius, 1803)

Recorded: Dobrogea (without other data) (Gruev, 2001); Romanian Dobrogea (Gruev, 2005).

Distribution: Palaearctic region; introduced in S Africa and North America.

Phyllotreta undulata (Kutschera, 1860)

Recorded: Măcin Mountains (Montandon, 1908); Slava Rusă (Crişan, 1993); Mamaia (Serafim \& Maican, 2004); Dobrogea (without other data) (Gruev, 2001; Skolka et al., 2005); Romanian Dobrogea (Gruev, 2005); Măcin, Greci, Pricopan (Skolka et al., 2006-2007).

Distribution: Palaearctic area; introduced in America and Australian region.

Phyllotreta variipennis variipennis (Boieldieu, 1859)

Recorded: Babadag (Montandon, 1887; Fleck, 1905 b); Romanian Dobrogea (Gruev, 2005).

Distribution: Canary Islands, Algeria, Iberian Peninsula, France, Italy, Switzerland, Balkan Peninsula, Asia Minor, Israel, Iran.

Phyllotreta vittula (L. Redtenbacher, 1849)

Recorded: Greci (Montandon, 1908); Gorgova, Maliuc, Babadag, Enisala (Crişan, 1993); Dobrogea (without other data) (Gruev, 2001; Skolka et al., 2005); Romanian Dobrogea (Gruev, 2005); Măcin, Greci, Pricopan (Skolka et al., 2006-2007)

Distribution: transpalaearctic species, distributed from Spain and Ireland to Korea; introduced to USA.

\section{Podagrica fuscicornis (Linnaeus, 1767)}

Recorded: Babadag (Fleck, 1905 b); Periprava (Negru, 1968 a); Caraorman sand bank (Ieniştea, 1974; Crişan, 1993); Maliuc, Gorgova, Enisala (Crişan, 1993); Beştepe, Letea Forest, Gorgova (Crişan, 1994); Sfântu Gheorghe, Dunavăț, Periteaşca, Lupilor sand bank (Sinoe Lake), Sacalin (Crişan, 1995 a); Comarova Forest (Mangalia) (Serafim \& Maican, 2004); Dobrogea (without other data) (Gruev, 2001; Skolka et al., 2005); Romanian Dobrogea (Gruev, 2005).

Material: 1 spec., Babadag, leg. Eduard Fleck.

Distribution: Central Europe, S Europe, Canary Islands, North Africa, Asia Minor, Near East.

Podagrica malvae malvae (Illiger, 1807)

Recorded: Comarova Forest (Mangalia) (Fleck, 1905 b; Negru, 1957; Negru \& Roşca, 1967); Greci (Montandon, 1908; Ionescu-Konnerth, 1963); Măcin, Tulcea, Niculițel (Roşca, 1976); Maliuc, Slava Rusă, Babadag (Crişan, 1993); at the intersection of Sulina arm with Magearu Channel (Crişan, 1994); Dunavăţ, Sacalin, Sfântu Gheorghe (Crișan, 1995 a); Dobrogea (without other data) (Gruev, 2001; Skolka et al., 2005); Romanian Dobrogea (Gruev, 2005); Măcin, Greci, Pricopan (Skolka et al., 2006-2007).

Material: Canaraua Fetii (Băneasa), 20.V.1993.

Distribution: Europe, North Africa, Asia Minor, Near East, the Caucasus, Iraq, Iran. Podagrica menetriesii (Faldermann, 1837)

Recorded: Tulcea (Gruev, 1983; Gruev et al., 1993); Dobrogea (without other data) (Gruev, 2001); Romanian Dobrogea (Gruev, 2005).

Material: Hagieni Forest, 22.V.1993.

Distribution: S and central part of Europe, Asia Minor, the Caucasus, Central Asia, W China.

Psylliodes (Psylliodes) affinis (Paykull, 1799)

Recorded: Greci (Montandon, 1908); Dobrogea (without other data) (Gruev, 2001); Romanian Dobrogea (Gruev, 2005). 
Distribution: Europe, Asia Minor, the Caucasus, Near East, Central Asia, Morocco. Psylliodes (Psylliodes) attenuata (Koch, 1803)

Recorded: Greci (Montandon, 1908); at the intersection of Sulina arm with Magearu Channel, Gorgova (Crişan, 1994); Dobrogea (without other data) (Gruev, 2001; Skolka et al., 2005); Romanian Dobrogea (Gruev, 2005); Măcin, Greci, Pricopan (Skolka et al., 2006-2007).

Material: Canaraua Fetii (Băneasa), 19.V.1993, Dumbrăveni Forest, 18.V.1994.

Distribution: transpalaearctic species, distributed from Ireland and Pyrenees to Japan.

\section{Psylliodes (Psylliodes) chalcomera (Illiger, 1807)}

Recorded: Dobrogea (without other data) (Gruev, 2001); Romanian Dobrogea (Gruev, 2005).

Material: Canaraua Fetii (Băneasa), 19.V.1993.

Distribution: transpalaearctic species distributed from Morocco and Great Britain to Russian Far East.

Psylliodes (Psylliodes) chrysocephala chrysocephala (Linnaeus, 1758)

(syn: cyanoptera Illiger, 1807)

Recorded: Pricopan (Jaquet, 1900 a; Fleck, 1905 b); Tulcea (Fleck, 1905 b); Babadag (Crişan, 1993); Beştepe (Crişan, 1994); Popina Island (Razim Lake), (Crişan, 1995 a); Dobrogea (without other data) (Gruev, 2001; Skolka et al., 2005); Romanian Dobrogea (Gruev, 2005); Măcin, Greci, Pricopan (Skolka et al., 2006-2007).

Distribution: Mediterraneana area, almost whole Europe, Asia Minor, the Caucasus. Psylliodes (Psylliodes) circumdata (W. Redtenbacher, 1842)

Recorded: Gorgova (Crişan, 1994); Dobrogea (without other data) (Skolka et al., 2005).

Distribution: Mediterranean area, North Africa from Morocco to Libya.

Psylliodes (Psylliodes) cuprea (Koch, 1803)

Recorded: Mangalia (Gruev, 2001); Romanian Dobrogea (Gruev, 2005).

Distribution: Europe, North Africa, Asia Minor, Near East, Central Asia, Mongolia. Psylliodes (Psylliodes) dulcamarae (Koch, 1803)

Recorded: Sfântu Gheorghe (Crişan, 1995 a); Dobrogea (without other data) (Skolka et al., 2005).

Distribution: Europe, Asia.

Psylliodes (Psylliodes) hyosciami (Linnaeus, 1758)

Recorded: Mangalia (Jaquet, 1900 a; Fleck, 1905 b; Montandon, 1908; IonescuKonnerth, 1963; Negru \& Roşca, 1967); Doloşman Cape (Razim Lake) (Crişan, 1993); Dobrogea (without other data) (Gruev, 2001; Skolka et al., 2005); Romanian Dobrogea (Gruev, 2005).

Distribution: transpalaearctic species, distributed from the British Isles to Russian Far East and in European part of Mediterranean area.

Psylliodes (Psylliodes) laticollis Kutschera, 1864

(syn: weberi Lohse, 1955)

Recorded: Beştepe (Crişan, 1994); Sfântu-Gheorghe (Crişan, 1995 a); Dobrogea (without other data) (Skolka et al., 2005).

Distribution: W Europe and North Africa, E Germany, N Italy, Greece and Crete; reported also from Slovakia and Bulgaria.

Psylliodes (Psylliodes) napi (Fabricius, 1792)

Recorded: Lupilor sand bank (Sinoe Lake) (Crişan, 1995 a); Dobrogea (without other data) (Skolka et al., 2005).

Distribution: Europe, Asia Minor, the Caucasus, Kazakhstan. 
Psylliodes (Psylliodes) picina (Marsham, 1802)

Recorded: Dobrogea (without other data) (Gruev, 2001); Romanian Dobrogea (Gruev, 2005).

Distribution: from Pyrenees and Ireland to S Finland and Black Sea; reported also from Spain.

\section{Psylliodes tricolor Weise, 1888}

(syn: sophiae Heikertinger, 1914)

Recorded: Beştepe, Gorgova (Crişan, 1994); Dunavăţ (Crişan, 1995 a); Dobrogea (without other data) (Gruev, 2001; Skolka et al., 2005); Romanian Dobrogea (Gruev, 2005).

Distribution: Europe, Asia Minor, the Caucasus, Near East, Central Asia; known also from Morocco.

Subfamily Lamprosomatinae Lacordaire, 1848

Oomorphus (Oomorphus) concolor (Sturm, 1807)

Recorded: Enisala (Crişan, 1993); at the intersection of Sulina arm with Magearu Channel (Crişan, 1994); Dobrogea (without other data) (Skolka et al., 2005).

Distribution: Europe from Pyrenees and Great Britain to Romania, reported also from Ukraine and the Caucasus.

Subfamily Cryptocephalinae Gyllenhal, 1813

Tribe Clytrini Kirby, 1837

Subtribe Clytrina Kirby, 1837

Clytra (Clytra) laeviuscula (Ratzeburg, 1837)

Recorded: Hagieni Forest (Negru, 1957); Canaraua Fetii (Băneasa) (Negru \& Roşca, 1967); Periprava, C.A. Rosetti (Negru, 1968 a); Măcin (Montandon, 1906; Roşca, 1976); Cocoş Monastery (Roşca, 1976); Beştepe, Letea Forest (Crişan, 1994); Olimp resort (North Mangalia) (Serafim \& Maican, 2004); Dobrogea (without other data) (Skolka et al., 2005); Romanian Dobrogea (Gruev, 2005); Băneasa Forest, Dumbrăveni Forest, Hagieni Forest, Canaraua Fetii (Băneasa), Babadag Forest, Slava Rusă, Cocoş Monastery, Letea Forest, Haşmacul lui Omer, Periprava, Caraorman (the Danube Delta) (Maican, 2006 a); Hagieni Forest (Maican, 2006 b); Măcin (Skolka et al., 2006-2007).

Material: 2 specs, Cernavodă, leg. Arnold Lucien Montandon; 2 specs, Măcin, leg. Arnold Lucien Montandon; 1 spec., Hagieni Forest, 12.VII.1992, leg. Rodica Serafim; 1 spec., Letea Forest, 18.VI.1993, leg. Ioana Matache.

Distribution: from France and Great Britain to Altai Range.

Clytra (Clytra) quadripunctata (Linnaeus, 1758)

Recorded: Esechioi Forest (Maican, 2006 a).

Distribution: W Palaearctic species, distributed from N Spain and Ireland to Mongolia.

Clytra (Clytraria) atraphaxidis atraphaxidis (Pallas, 1773)

Recorded: Mangalia (Montandon, 1887; Fleck, 1905 b); Pricopan Crest near Măcin (Jaquet, 1900 a; Fleck, 1905 b); Măcin (Montandon, 1906; Roşca, 1976; Maican, 2006 a; Skolka et al., 2006-2007); Greci (Montandon, 1908); Canaraua Fetii (Negru \& Roşca, 1967); Pricopan (Roşca, 1976; Skolka et al., 2006-2007); Dobrogea (without other data) (Skolka et al., 2005); Romanian Dobrogea (Gruev, 2005); Hagieni Forest; Somova (Maican, 2006 a).

Material: 10 specs, Canaraua Fetii, 11-14.VI.1960, leg. Nicolae Săvulescu. 
Distribution: from Spain, Corsica. Sicily, S Italy and Greece to Central Asia, Mongolia and Korea.

$$
\text { Clytra (Clytraria) novempunctata Olivier, } 1808
$$

Recorded: Iortmac, Băneasa Forest, Canaraua Fetii (Băneasa), Dobromir Forest (Maican, 2006 a).

Distribution: SE Europe, the Caucasus, Asia Minor, Central Asia; reported also from Sicily.

\section{Clytra (Clytraria) valeriana valeriana (Ménétries, 1832)}

Recorded: Pricopan Crest near Măcin (Jaquet, 1900 a; Fleck, 1905 b); Romanian Dobrogea (Gruev, 2005); Măcin, Greci, Pricopan (Skolka et al., 2006-2007).

Distribution: Greece, Serbia, Bulgaria, the Caucasus, Middle East, Central Asia; known also from Sicily.

Coptocephala gebleri Gebler, 1841

Recorded: Cernavodă (Hurmuzachi, 1904); Mangalia, Constanţa (Fleck, 1905 b); Letea Forest (Ieniştea, 1968); Caraorman (Ieniştea, 1974); Hagieni Forest, Istria; Romanian Dobrogea (Gruev, 2005); Periprava, Caraorman (Maican, 2006 a).

Distribution: N Greece, Bulgaria, Crimea, S Russia.

Coptocephala unifasciata unifasciata (Scopoli, 1763)

Recorded: Mangalia (Fleck, 1905 b); Hagieni Forest (Negru \& Roşca, 1967); Periprava (Negru, 1968 a); Letea Forest (Ieniştea, 1968); Caraorman (Ieniştea, 1974); Babadag, Enisala, Caraorman, Histria (Crişan, 1993); Sfântu Gheorghe (Crişan, 1995 a); Agigea (Serafim \& Maican, 2004); Dobrogea (without other data) (Skolka et al., 2005); Romanian Dobrogea (Gruev, 2005).

Distribution: very widely distributed from N Spain and Belgium to Middle East, Central Asia and Mongolia.

Labidostomis (Labidostomis) cyanicornis Germar, 1822

Recorded: Comarova Forest (Mangalia) (Negru, 1957; Negru \& Roşca, 1967); Dobrogea (without other data) (Skolka et al., 2005); Romanian Dobrogea (Gruev, 2005).

Distribution: S part of Central Europe, Romania, Ukraine, basin of Volga.

Labidostomis (Labidostomis) humeralis (D. H. Schneider, 1792)

Recorded: Băneasa Forest, Canaraua Fetii (Băneasa) (Maican, 2006 a).

Distribution: from central France and S Sweden to Asia Minor and basin of Volga.

Labidostomis (Labidostomis) longimana (Linnaeus, 1760)

Recorded: Tuzla (Serafim \& Maican, 2004); Dobrogea (without other data) (VasiliuOromulu et al., 2004); Luncaviţa, Cetăţuie, Valu lui Traian, Babadag Forest, Băneasa Forest, Esechioi Forest; Crişan (Maican, 2006 a).

Distribution: from S Italy, N Spain and S Sweden to central Russia and Mongolia. Labidostomis (Labidostomis) lucida (Germar, 1824)

Recorded: Mangalia, Techirghiol Lake (Jaquet, 1899; Fleck, 1905 b); Comarova Forest (Mangalia) (Negru \& Roşca, 1967); Măcin (Montandon, 1906); Dobrogea (without other data) (Skolka et al., 2005); Romanian Dobrogea (Gruev, 2005); Dobrogea, Hagieni Forest, Agigea (Maican, 2006 a); Măcin (Skolka et al., 20062007).

Material: 1 spec., Valu lui Traian, 5.VI.1954, leg. Xenia Scobiola Palade.

Distribution: Europe.

Labidostomis (Labidostomis) pallidipennis (Gebler, 1830)

Recorded: Olimp resort (North Mangalia) (Serafim \& Maican, 2004; Maican, 2006 a); Hagieni Forest, Agigea (Maican, 2006 a).

Distribution: from N Spain, Italy and basin of Danube to China. 
Labidostomis (Labidostomis) propinqua propinqua Faldermann, 1837

Recorded: Hagieni Forest, Băneasa Forest, Canaraua Fetii (Băneasa) (Maican, 2006 a).

Distribution: Bulgaria, S Romania, Greece, Asia Minor, the Caucasus.

Lachnaia (Lachnaia) sexpunctata (Scopoli, 1763)

Recorded: Măcin (Montandon, 1906); Hagieni Forest (Negru \& Roșca, 1967); Canaraua Fetii (Băneasa) (Balog, 1998); Dobrogea (without other data) (Skolka et al., 2005); Romanian Dobrogea (Gruev, 2005); Babadag Forest, Hagieni Forest, Băneasa Forest, Dumbrăveni Forest, Canaraua Fetii, Valu lui Traian, Niculiţel; Caraorman (Maican, 2006 a).

Material: Dumbrăveni Forest, 18.V.1994.

Distribution: NE France, S Germany, basin of Danube, Romania, Ukraine, Balkan Peninsula, Asia Minor.

Smaragdina aurita aurita (Linnaeus, 1767)

Recorded: Babadag (Crişan, 1993); Dobrogea (without other data) (Skolka et al., 2005); Babadag Forest; Caraorman (Maican, 2006 a).

Distribution: Pyrenees, W France, Italy, Central Europe, reported also from the Caucasus.

Smaragdina flavicollis Charpentier, 1825

Recorded: Canaraua Fetii (Băneasa) (Balog, 1998).

Distribution: Europe from France and N Italy to Ukraine, N Turkey; the Caucasus, Lithuania and S Finland.

\section{Smaragdina limbata (Stéven, 1806)}

Recorded: Ciucurova, Babadag (Fleck, 1905 b); Măcin (Montandon, 1906); Comarova Forest (Mangalia) (Negru, 1957; Negru \& Roșca, 1967); Canaraua Fetii (Băneasa) (Balog, 1998); Neptun resort (North Mangalia) (Serafim \& Maican, 2004); Dobrogea (without other data) (Skolka et al., 2005); Romanian Dobrogea (Gruev, 2005); Hagieni Forest, Mangalia, Comarova Forest (Mangalia), Istria, Băneasa, Canaraua Fetii (Băneasa), Limanu, Dumbrăveni Forest (Maican, 2006 a).

Material: Canaraua Fetii (Băneasa), 20.V.1993, leg. Cristina Hoinic; Hagieni Forest, 22.V.1993, leg. Angela Petrescu.

Distribution: Mediterranean species, distributed in Balkan Peninsula, Asia Minor, the Caucasus, Middle East, N Iran.

Smaragdina salicina (Scopoli, 1763)

(syn: cyanea Fabricius, 1775)

Recorded: Pricopan Crest near Măcin (Jaquet, 1900 a; Fleck, 1905 b); Măcin Mountains (Montandon, 1908); Comarova Forest (Mangalia) (Negru \& Roşca, 1967; Serafim \& Maican, 2004; Maican, 2006 a); Dobrogea (without other data) (Skolka et al., 2005); Romanian Dobrogea (Gruev, 2005); Băneasa Forest, Canaraua Fetii (Băneasa), Mangalia, Caraorman (Maican, 2006 a); Dumbrăveni Forest (Maican, 2006 b).

Distribution: Central and S Europe from N Spain and Denmark to basin of Volga and the Caucasus.

Smaragdina xanthaspis (Germar, 1824)

Recorded: Măcin (Fleck, 1905 b); Comarova Forest (Mangalia), Hagieni Forest (Negru \& Roşca, 1967); Comarova Forest (Mangalia), Agigea, Neptun resort (Mangalia Nord) (Serafim \& Maican, 2004); Dobrogea (without other data) (Skolka et al., 2005); Romanian Dobrogea (Gruev, 2005); Mangalia, Comarova Forest (Mangalia), Hagieni Forest, Dumbrăveni Forest (Maican, 2006 a); Material: Dumbrăveni Forest, 18.V.1994. 
Distribution: N Italy, N part of Balkan Peninsula, basin of Danube, S Ukraine, Asia Minor.

Tituboea macropus (Illiger, 1800)

Recorded: Sfântu Gheorghe (Fleck, 1905 b; Ieniştea, 1974); Periprava, C.A. Rosetti (Negru, 1968 a); Caraorman, (Ieniştea, 1974); Măcin Mountains, Greci (Roşca, 1976); Romanian Dobrogea (Gruev, 2005); Sfântu Gheorghe, Sulina, Caraorman, Periprava, Letea Forest, C.A. Rosetti, Limanu, Hagieni Forest, Măcin, Greci (Maican, 2006 a); Măcin, Greci (Skolka et al., 2006-2007).

Material: 2 specs, Sfântu Gheorghe, leg. Arnold Lucien Montandon.

Distribution: SE Europe and Asia Minor, from Austria and Albania to basin of Volga and the Caucasus.

Tribe Cryptocephalini Gyllenhal, 1813

Subtribe Cryptocephalina Gyllenhal, 1813

Cryptocephalus (Asionus) apicalis Gebler, 1830

Recorded: Mangalia (Jaquet, 1899; Fleck, 1905 b; Negru \& Roşca, 1967); Pricopan, Babadag, Constanţa (Fleck, 1905 b; Negru \& Roşca, 1967); Măcin Mountains (Montandon, 1906; Skolka et al., 2006-2007); Agigea, Caraorman (Roşca, 1973); Enisala, Babadag (Crişan, 1993); Beştepe, Agighiol, Iancina Cape (Crişan, 1994); Canaraua Fetii (Băneasa) (Roșca, 1973; Balog, 1998); Agigea, Comarova Forest (Mangalia) (Serafim \& Maican, 2004); Fântâniţa Nature Reserve (Vasiliu-Oromulu et al., 2004); Dobrogea (without other data) (Skolka et al., 2005); Romanian Dobrogea (Gruev, 2005); Greci, Pricopan (Skolka et al., 2006-2007); Visterna, Babadag (Serafim \& Maican, 2011).

Material: 2 specs, Măcin, leg. Arnold Lucien Montandon; 1 spec., Mangalia, leg. Eduard Fleck; 1 spec., 19.V.1993, Canaraua Fetii, leg. Gabriela Andrei; Iancina Cape (Razim Lake), 11.VI.1993, leg. Ioana Matache; Beştepe, 13.VI.1993, leg. Ioana Matache.

Distribution: from Austria to E Siberia; in Europe known mostly from Slovakia, Balkans, Ukraine.

\section{Cryptocephalus (Asionus) bohemius Drapiez, 1819}

Recorded: Letea Forest, Periprava (Roşca, 1973); Romanian Dobrogea (Gruev, 2005).

Material: Caraorman, 25.VI.1991, leg. Nicolae Găldean; Letea Forest, 18.VI.1993, leg. Ioana Matache.

Distribution: Austria, Hungary, Ukraine, S Russia, Siberia, Mongolia.

Cryptocephalus (Asionus) flexuosus Krynicki, 1834

Recorded: Romanian Dobrogea (Gruev, 2005).

Distribution: Bulgaria, Asia Minor, E Ukraine, Caucasian countries, Central Asia.

Cryptocephalus (Asionus) gamma Herrich-Schäffer, 1835

Recorded: Periteaşca, Gura Portiţei, Sfântu Gheorghe (Crişan, 1995 a, b); Dobrogea (without other data) (Skolka et al., 2005).

Distribution: Slovakia, Hungary, Bulgaria, Romania, Ukraine, basin of Volga.

Cryptocephalus (Asionus) quatuordecimmaculatus D. H. Schneider, 1792

Recorded: Cernavodă, Murfatlar (Fleck, 1905 b); Fântâniţa Nature Reserve (VasiliuOromulu et al., 2004).

Distribution: basin of Danube, Bulgaria, Ukraine.

Cryptocephalus (Burlinius) bilineatus (Linnaeus, 1767)

Caraorman (Crişan, 1993); Dobrogea (without other data) (Skolka et al., 2005).

Distribution: transpalaearctic species, very widely distributed. 
Cryptocephalus (Burlinius) chrysopus (Gmelin, 1790)

Recorded: Romanian Dobrogea (Gruev, 2005); Măcin, Greci, Pricopan (Skolka et al., 2006-2007).

Distribution: Central Europe, S France, N Italy, Bulgaria, Crimea.

Cryptocephalus (Burlinius) connexus Olivier, 1808

Recorded: Greci (Montandon, 1908); C.A. Rosetti (Ieniştea, 1968); Letea Forest, Valu lui Traian, Periprava (Roşca, 1973); Histria, Caraorman, Enisala, Babadag (Crişan, 1993); Sfântu Gheorghe, Lupilor sand bank (Sinoe Lake) (Crişan, 1995 a); Tuzla, Comarova Forest (Mangalia), Agigea Natural Reserve (Serafim \& Maican, 2004); Dobrogea (without other data) (Skolka et al., 2005); Romanian Dobrogea (Gruev, 2005); Măcin, Greci, Pricopan (Skolka et al., 2006-2007).

Distribution: S Europe, Asia Minor, Central Asia.

Cryptocephalus (Burlinius) frontalis Marsham, 1802

Recorded: Beştepe (Crişan, 1994); Dobrogea (without other data) (Skolka et al., 2005).

Distribution: Central Europe and S part of Fennoscandia.

Cryptocephalus (Burlinius) fulvus fulvus Goeze, 1777

Recorded: Letea Forest (Crişan, 1994); Dobrogea (without other data) (Skolka et al., 2005).

Distribution: Palaearctic species, very videly distributed.

Cryptocephalus (Burlinius) labiatus (Linnaeus, 1761)

Recorded: Greci (Montandon, 1908); Romanian Dobrogea (Gruev, 2005); Măcin, Greci, Pricopan (Skolka et al., 2006-2007).

Distribution: widely distributed European species.

Cryptocephalus (Burlinius) ocellatus ocellatus Drapiez, 1819

Recorded: Greci (Montandon, 1908); Beştepe, Letea Forest (Crişan, 1994); Dunavăţ (Crişan, 1995 a); Dobrogea (without other data) (Skolka et al., 2005); Romanian Dobrogea (Gruev, 2005).

Distribution: Palaearctic species.

Cryptocephalus (Burlinius) populi Suffrian, 1848

Recorded: Dunavăţ (Crişan, 1995 a); Dobrogea (without other data) (Skolka et al., 2005).

Distribution: distributed in a great part of Europe.

Cryptocephalus (Burlinius) pusillus Fabricius, 1777

Recorded: Valea Fagilor Forest (Skolka et al., 2006-2007).

Distribution: distributed in almost whole Europe.

Cryptocephalus (Burlinius) querceti Suffrian, 1848

Recorded: Mangalia (Fleck, 1905 b; Negru \& Roșca, 1967; Roșca, 1973); Letea Forest (Crişan, 1994); Dobrogea (without other data) (Skolka et al., 2005); Romanian Dobrogea (Gruev, 2005).

Distribution: Central and N Europe.

Cryptocephalus (Cryptocephalus) androgyne androgyne Marseul, 1875

(syn.: coerulescens R. C. Sahlberg, 1839)

Recorded: Dunavăţ, Sfântu Gheorghe (Crişan, 1995 a); Dobrogea (without other data) (Skolka et al., 2005).

Distribution: central and N part of Europe.

Cryptocephalus anticus Suffrian, 1848

(syn: octacosmus Bedel, 1891)

Recorded: Greci (Montandon, 1908); Periprava (Ieniştea, 1968); Luncaviţa Cetăţuie (Măcin Mountains), Periprava, Letea Forest (Roşca, 1973); Caraorman (Ieniştea, 
1974); Tulcea, Măcin Mountains, Greci (Roșca, 1976); Caraorman, Maliuc (Crișan, 1993); Gorgova, at the intersection of Sulina arm with Magearu Channel (Crişan, 1994); Sacalin, Lupilor sand bank (Sinoe Lake), Dunavăţ (Crişan, 1995 a); Dobrogea (without other data) (Skolka et al., 2005); Romanian Dobrogea (Gruev, 2005); Măcin, Greci (Skolka et al., 2006-2007); C.A. Rosetti, Danube Delta (Serafim \& Maican, 2011).

Material: Dunavăț, 9.VI.1994.

Distribution: Central and S Europe, from N Spain and Belgium to Greece, Asia Minor and basin of Volga.

Cryptocephalus (Cryptocephalus) aureolus aureolus Suffrian, 1847

Recorded: Horoslar (Hurmuzachi, 1904); Babadag (Fleck, 1905 b); C.A. Rosetti (Negru, 1968 a); Romanian Dobrogea (Gruev, 2005).

Distribution: from Spain and Great Britain to Russia, the Caucasus, Central Asia.

Cryptocephalus (Cryptocephalus) bipunctatus bipunctatus (Linnaeus, 1758)

Recorded: Pricopan (Fleck, 1905 b); Măcin Mountains, Turcoaia (Iglița) Lake (Montandon, 1908); Comarova Forest (Mangalia), Hagieni Forest, Babadag, Caraorman (Negru \& Roşca, 1967; Roşca, 1973); Greci, Măcin Mountains, Pricopan (Roșca, 1976; Serafim \& Maican, 2004); Dobrogea (without other data) (VasiliuOromulu et al., 2004; Skolka et al., 2005); Romanian Dobrogea (Gruev, 2005); Hagieni Forest, Dumbrăveni Forest (Maican, 2006 b); Visterna, Babadag (Serafim \& Maican, 2011).

Material: 3 specs, Măcin, leg. Arnold Lucien Montandon; 1 spec., Mangalia, leg. Eduard Fleck; Canaraua Fetii (Băneasa), 20.V.1994.

Distribution: transpalaearctic species, very videly distributed, from Portugal and Ireland to Korea.

Cryptocephalus (Cryptocephalus) cordiger (Linnaeus, 1758)

Recorded: Visterna, Babadag (Serafim \& Maican, 2011).

Distribution: from E France and S Sweden to E Siberia.

Cryptocephalus (Cryptocephalus) duplicatus Suffrian, 1847

Recorded: Romanian Dobrogea (Gruev, 2005).

Distribution: E part of Balkan Peninsula, Asia Minor.

Cryptocephalus (Cryptocephalus) flavipes Fabricius, 1781

Recorded: Mangalia, Constanţa (Fleck, 1905 b; Negru \& Roşca, 1967); Comarova Forest (Mangalia), Agigea (Serafim \& Maican, 2004); Dobrogea (without other data) (Skolka et al., 2005); Romanian Dobrogea (Gruev, 2005); Eforie Nord (Nagy, 1972); Dumbrăveni Forest (Maican, 2006 b).

Material: Canaraua Fetii (Băneasa), 20.V.1994.

Distribution: Europe (except Iberian Peninsula, British Island and N Scandinavia), Asia Minor, Russia, the Caucasus, Central Asia to Altai.

Cryptocephalus (Cryptocephalus) hypochoeridis (Linnaeus, 1758)

Recorded: Babadag (Fleck, 1905 b); Greci, Niculiţel (Roşca, 1976); Romanian Dobrogea (Gruev, 2005).

Distribution: most part of Europe, the Caucasus, Central Asia.

Cryptocephalus (Cryptocephalus) imperialis Laicharting, 1781

Recorded: Hagieni Forest (Negru \& Roşca, 1967); Comarova Forest (Mangalia) (Roşca, 1973; Serafim \& Maican, 2004); Greci (Roşca, 1976); Beştepe (Crişan, 1994); Olimp Resort (North Mangalia), Agigea Natural Reserve (Serafim \& Maican, 2004); Romanian Dobrogea (Gruev, 2005).

Distribution: N Spain, France, South Germany, basin of Danube, Balkan Peninsula, Romania, Ukraine, Asia Minor. 
Cryptocephalus (Cryptocephalus) janthinus Germar, 1824

Recorded: Caraorman (Ieniștea, 1974); at the intersection of Sulina arm with Magearu Channel (Crişan, 1994); Dobrogea (without other data) (Skolka et al., 2005); C.A. Rosetti, Danube Delta (Serafim \& Maican, 2011).

Distribution: France, Italy, Central Europe, Romania, Bulgaria, Ukraine, S Russia, the Caucasus.

Cryptocephalus (Cryptocephalus) laetus Fabricius, 1792

Recorded: Cernavodă (Hurmuzachi, 1904; Fleck, 1905 b); Letea Forest (Ieniștea, 1968); Caraorman (Crişan, 1993); Agigea (Serafim \& Maican, 2004); Dobrogea (without other data) (Skolka et al., 2005); Romanian Dobrogea (Gruev, 2005).

Distribution: basin of Danube, Poland, Romania, Ukraine, S Russia, the Caucasus.

Cryptocephalus (Cryptocephalus) moraei (Linnaeus, 1758)

Recorded: Mangalia, Agigea, Babadag, Valu lui Traian (Roşca, 1973); Enisala (Crişan, 1993); Beştepe, Gorgova, Letea Forest (Crişan, 1994); Dobrogea (without other data) (Skolka et al., 2005); Romanian Dobrogea (Gruev, 2005); Dumbrăveni Forest (Maican, 2006 b); Visterna, Babadag (Serafim \& Maican, 2011).

Material: 1 spec., Babadag, leg. Eduard Fleck; Letea Forest, Beştepe, 13-18.VI.1993, leg. Ioana Matache.

Distribution: Europe (except N part of Scandinavia), reported from Asia Minor.

Cryptocephalus (Cryptocephalus) quadriguttatus C. F. W. Richter, 1820

Recorded: Beştepe (Crişan, 1994).

Distribution: basin of Danube, Romania, SE Poland, Ukraine, S Russia, the Caucasus.

Cryptocephalus (Cryptocephalus) quinquepunctatus (Scopoli, 1763)

Recorded: Hagieni (Negru, 1957; Negru \& Roşca, 1967); Romanian Dobrogea (Gruev, 2005).

Distribution: Central part of Europe, from NE Italy and Belgium to Ukraine.

Cryptocephalus (Cryptocephalus) sericeus (Linnaeus, 1758)

Recorded: Horoslar (Hurmuzachi, 1904); Mangalia, Babadag (Fleck, 1905 b; Negru \& Roşca, 1967); Hagieni Forest (Negru, 1957); Comarova Forest (Mangalia) (Negru \& Roşca, 1967); Periprava, C.A. Rosetti (Negru, 1968 a); Valu lui Traian, Caraorman, Agigea, Periprava, Techirghiol (Roşca, 1973); Histria, Caraorman (Crișan, 1993); Beștepe, Agighiol (Crișan, 1994); Sfântu Gheorghe (Crişan, 1995 a); Agigea, Comarova Forest (Mangalia) (Serafim \& Maican, 2004); Dobrogea (without other data) (Skolka et al., 2005); Romanian Dobrogea (Gruev, 2005).

Material: 1 spec., Babadag, 20.VI.1958, leg. Nicolae Săvulescu; 1 spec., South of Dobrogea, 04.VI.1958, leg. Nicolae Săvulescu; Canaraua Fetii (Băneasa), 20.V.1994. Distribution: from N Spain, Belgium and Denmark to NW China.

Cryptocephalus (Cryptocephalus) sexpunctatus sexpunctatus (Linnaeus, 1758) Recorded: Greci (Roşca, 1976); Romanian Dobrogea (Gruev, 2005; Skolka et al., 2006-2007).

Distribution: central and N part of Europe, from Great Britain, E France, N Italy and basin of the Danube to the Arctic Circle.

Cryptocephalus (Cryptocephalus) trimaculatus Rossi, 1790

Recorded: Comarova Forest (Mangalia), Hagieni (Negru \& Roșca, 1967); Dobrogea (without other data) (Skolka et al., 2005); Romanian Dobrogea (Gruev, 2005).

Distribution: S part of Europe from S France and Italy to Hungary, Balkan Peninsula, Asia Minor, Near East.

Cryptocephalus (Cryptocephalus) turcicus Suffrian, 1847

Recorded: Babadag, Constanţa (Fleck, 1905 b); Romanian Dobrogea (Gruev, 2005). Distribution: S Europe from France to Bulgaria and in Asia Minor. 
Cryptocephalus (Cryptocephalus) violaceus violaceus Laicharting, 1781

Recorded: Mangalia (Jaquet, 1900 a; Fleck, 1905 b); Cocoş Monastery, Orliga (Măcin Mountains) (Jaquet, 1900 a; Fleck, 1905 b); Măcin Mountains (Montandon, 1908); Babadag (Crişan, 1993); Canaraua Fetii (Băneasa) (Balog, 1998); Romanian Dobrogea (Gruev, 2005); Măcin, Greci, Pricopan (Skolka et al., 2006-2007).

Material: 1 spec., Babadag, leg. Eduard Fleck; Canaraua Fetii (Băneasa), 20.V.1994. Distribution: W, central and SE Europe from Spain to Ukraine and Asia Minor.

Cryptocephalus (Cryptocephalus) virens Suffrian, 1847

Recorded: Orliga (Măcin Mountains) (Jaquet, 1900 a; Fleck, 1905 b).

Distribution: SE Europe fom Italian Alps and Bavaria to Balkan Peninsula, S Russia, Asia Minor.

Cryptocephalus (Cryptocephalus) vittatus Fabricius, 1775

Recorded: Romanian Dobrogea (Gruev, 2005).

Distribution: Iberian Peninsula, France, Central Europe, basins of Danube and Dnieper.

Cryptocephalus (Heterichnus) coryli (Linnaeus, 1758)

Recorded: Dobrogea (without other data) (Roşca, 1973); Romanian Dobrogea (Gruev, 2005).

Distribution: transpalaearctic species, distributed from the British Isles to Korea.

Cryptocephalus (Protophysus) schaefferi schaefferi Schrank, 1789

Recorded: Babadag (Fleck, 1905 b; Roșca, 1973); Romanian Dobrogea (Gruev, 2005).

Distribution: S and SE Europe, from N Spain to basin of Volga, in Asia Minor and the Caucasus.

Subtribe Pachybrachina Chapuis, 1874

Pachybrachis (Pachybrachis) fimbriolatus (Suffrian, 1848)

Recorded: Mangalia (Jaquet, 1900 a; Fleck, 1905 b; Negru \& Roşca, 1967); at the intersection of Sulina arm with Magearu Channel (Crişan, 1994); Agigea Natural Reserve (Serafim \& Maican, 2004); Dobrogea (without other data) (Skolka et al., 2005); Romanian Dobrogea (Gruev, 2005).

Distribution: S and central part of Europe from N Spain to Asia Minor and basin of Volga.

\section{Pachybrachis (Pachybrachis) hieroglyphicus (Laicharting, 1781)}

Recorded: Mangalia (Negru \& Roşca, 1967); Periprava (Ieniştea, 1968); Agigea Natural Reserve (Serafim \& Maican, 2004); Dobrogea (without other data) (Skolka et al., 2005); Romanian Dobrogea (Gruev, 2005).

Distribution: very widely distributed from Iberian Peninsula to E Siberia.

Pachybrachis (Pachybrachis) hippophaes (Suffrian, 1848)

Recorded: Sfântu Gheorghe (Crişan, 1995 a); Dobrogea (without other data) (Skolka et al., 2005).

Distribution: from N Spain to Romania.

Pachybrachis (Pachybrachis) picus (Weise, 1882)

Recorded: Gura Portiţei (Crişan, 1995 a); Dobrogea (without other data) (Skolka et al., 2005).

Distribution: from N Spain, central France and Belgium to Hungary and central Italy.

Pachybrachis (Pachybrachis) sinuatus (Mulsant \& Rey, 1859)

Recorded: Gura Portiţei (Crişan, 1995 a); Dobrogea (without other data) (Skolka et al., 2005).

Distribution: S France, Central Europe, Balkans, Asia Minor. 
Pachybrachis (Pachybrachis) tesselatus tesselatus (Olivier, 1791)

Recorded: Beştepe, Letea Forest (Crişan, 1994); Dobrogea (without other data) (Skolka et al., 2005).

Distribution: S and central part of Europe from N Spain to the Caspian Sea.

Subtribe Stylosomina Chapuis, 1874

Stylosomus (Stylosomus) flavus flavus Marseul, 1875

Recorded: Dunavăţ, Periteaşca (Danube Delta) (Hoinic, 1995).

Distribution: Balkan Peninsula, Ukraine, S Russia.

Stylosomus (Stylosomus) tamaricis (Herrich-Schäffer, 1836)

Recorded: Sulina (Ieniştea, 1974); Dunavăț, Lupilor sand bank (Sinoe Lake), Sfântu Gheorghe (Crişan, 1995 a, b); Dobrogea (without other data) (Skolka et al., 2005).

Distribution: W part of the Mediterranean area from Morocco to Dalmatia and Central Asia.

Subfamily Eumolpinae Hope, 1840

Tribe Bromiini Chapuis, 1874

Macrocoma rubripes rubripes (Schaufuss, 1862)

Recorded: Mangalia, Techirghiol Lake (Jaquet, 1900 a; Fleck, 1905 b; Montandon, 1908; Negru \& Roşca, 1967); Dobrogea (without other data) (Skolka et al., 2005).

Distribution: Balkan Peninsula, Cyprus, Asia Minor, Syria, the Caucasus.

Macrocoma rubripes balcanica Apfelbeck, 1912

Recorded: Romanian Dobrogea (Gruev, 2005).

Distribution: Balkan Peninsula, Cyprus, Asia Minor, Syria, Caucasian countries.

Pachnephorus (Pachnephorus) pilosus (Rossi, 1790)

(syn: arenarius Panzer, 1797)

Recorded: Isaccea (Fleck, 1905 b); Romanian Dobrogea (Gruev, 2005); Măcin, Greci, Pricopan (Skolka et al., 2006-2007).

Distribution: Europe, from W France and Belgium to S Finland, N Italy, Balkan Peninsula, Asia Minor.

Pachnephorus (Pachnephorus) tessellatus (Duftschmid, 1825)

Recorded: Cernavodă (Fleck, 1905 b).

Distribution: Europe, Central Asia.

Pachnephorus (Pachnephorus) villosus (Duftschmid, 1825)

(syn: aspericollis Fairmaire, 1862)

Recorded: Măcin Mountains, Isaccea (Montandon, 1887); Constanța, Isaccea (Fleck, 1905 b); Greci (Montandon, 1908); Maliuc (Crişan, 1993); Beştepe, at the intersection of Sulina arm with Magearu Channel (Crişan, 1994); Dunavăţ (Crişan, 1995 a); Mangalia (Nitzu, 2001); Dobrogea (without other data) (Skolka et al., 2005); Romanian Dobrogea (Gruev, 2005); Măcin, Greci, Pricopan (Skolka et al., 20062007).

Distribution: Italy, the Danube basin, Balkan Peninsula, Asia Minor, Ukraine, the Caucasus.

Chrysochus asclepiadeus asclepiadeus Pallas, 1773

Recorded: Sulina (Fleck, 1905 b); Sulina, Periprava, Letea Forest, C.A. Rosetti (Negru, 1968 a); Sfântu Gheorghe (Crişan, 1995 a); Dobrogea (without other data) (Skolka et al., 2005).

Material: 2 specs, Sulina, leg. Arnold Lucien Montandon; 1 spec., Canaraua Fetii, 29.VI.1956, leg. Nicolae Săvulescu.

Distribution: France, N Italy, S Germany, Poland, basins of Danube, Dnieper and Volga, the Caucasus, Central Asia. 
Eupales ulema (Germar, 1813)

Recorded: Constanța (Serafim \& Maican, 2004).

Distribution: Balkan Peninsula, Hungary.

\section{DISCUSSIONS}

Based on data obtained so far, the Cerambycidae, Chrysomelidae and Orsodacnidae fauna from Dobrogea indicates a high diversity, being recorded 407 species belonging to 132 genera and 15 subfamilies.

Among them, 139 species belong to Cerambycidae family, two species belong to Orsodacnidae family and 266 species belong to Chrysomelidae family.

The species Lilioceris lilii, Oulema erichsonii, Euluperus cyaneus and Altica quercetorum quercetorum are mentioned for the first time in fauna of Dobrogea.

The Dobrogea coleopterofauna is characterized by the presence of the following endemic species: Vadonia hirsuta (endemic in the Romanian fauna), Dorcadion equestre transsilvanicum (endemic in the Republic of Moldova and Romania), D. axillare (endemic in Romania and Bulgaria), D. gashtarovi (endemic in Dobrogea, including the Bulgarian side) and Brachyta balcanica (endemic in the Balkan Peninsula).

Among the faunal elements that bring high value to the biodiversity of this region we mention the rare species (Cryptocephalus bohemius, Clytra novempuncatata, C. valerianae, Macroplea mutica, Clytus tropicus, Cyrtoclytus capra, Deroplia genei, Phytoecia praetextata praetextata, Cerambyx miles, C. welensii, Vadonia moesiaca, Cortodera differens, Agapanthia kirbyi, Lampropterus femoratus, Rusticoclytus pantherinus, Plagionotus bobelayei, Pedostrangalia verticalis, Vadonia bipunctata globicollis, Coptosia albovittigera), Pontic and Mediterranean species (Coptocephala gebleri, Tituboea macropus etc.).

Macroplea mutica is an extremely rare halophilous species, threatened with extinction.

Trichoferus campestris is an Asian invasive species.

From conservation point of view, Rosalia alpina alpina (priority species), Morimus asper funereus, and Cerambyx cerdo cerdo are protected species, included in the annexes of the Habitats Directive 92/43/EEC. Also, Brachyta balcanica, Pedostrangalia verticalis and Neodorcadion exornatum are species of national interest requiring strict protection, listed in the Annex 4B from Government Emergency Ordinance no. 57/2007 on the regime of natural protected areas, the conservation of natural habitats, flora and fauna.

\section{ACKNOWLEDGEMENTS}

Special thanks to Dr. Maria Magdalena Dascălu (Faculty of Biology, "Al. I. Cuza” University, Iaşi, Romania) and Dr. Alexandru Crişan ("Babeş-Bolyai" University, Cluj-Napoca, Romania) for their constructive comments and publishing advice. The study of Chrysomelidae family was funded by project no. RO1567-IBB03/2012 from the Institute of Biology Bucharest of Romanian Academy.

PRIVIRE ASUPRA SUPRAFAMILIEI CHRYSOMELOIDEA (COLEOPTERA: CERAMBYCIDAE, ORSODACNIDAE, CHRYSOMELIDAE) INN DOBROGEA (ROMÂNIA)

\section{REZUMAT}

Lucrarea reprezintă o sinteză referitoare la diversitatea specifică a coleopterelor fitofage din suprafamilia Chrysomeloidea (familiile Cerambycidae, Orsodacnidae şi Chrysomelidae) în Dobrogea, realizată pe baza informaţiilor bibliografice şi a studiului materialului din colecţiile 
entomologice ale Muzeului Naţional de Istorie Naturală "Grigore Antipa" şi Institutului de Biologie Bucureşti al Academiei Române.

Sunt prezentate date de distribuție referitoare la 407 specii din suprafamilia Chrysomeloidea (dintre care, 139 specii Cerambycidae, două specii Orsodacnidae şi 266 specii Chrysomelidae), încadrate în 132 genuri, din 15 subfamilii.

Speciile Lilioceris lilii (Scopoli), Oulema erichsonii (Suffrian), Euluperus cyaneus (Joannis) şi Altica quercetorum quercetorum Foudras sunt menţionate pentru prima dată în fauna Dobrogei.

Se remarcă prezența speciilor endemice: Vadonia hirsuta (K. Daniel \& J. Daniel) (endemică în România), Dorcadion equestre transsilvanicum Ganglbauer (endemică în Republica Moldova şi România), Dorcadion axillare (endemică în România şi Bulgaria), Dorcadion gashtarovi Sama, Dascălu \& Pesarini (endemică în Dobrogea, inclusiv partea bulgărească) şi Brachyta balcanica Hampe (endemit balcanic).

Dintre speciile rare sunt menţionate: Coptosia albovittigera Heyden, Deroplia genei genei (Aragona), Phytoecia praetextata praetextata (Steven), Cerambyx miles Bonelli, Cerambyx welensii Kuster, Vadonia moesiaca Daniel \& Daniel, Cortodera differens Pic, Agapanthia kirbyi (Gyllenhal), Macroplea mutica (Fabricius), Clytra valeriana (Ménétries) and Cryptocephalus bohemius Drapiez.

Din punct de vedere conservativ, importante sunt speciile Rosalia alpina alpina (Linnaeus), Morimus asper funereus Mulsant, Cerambyx cerdo cerdo Linnaeus, Pilemia tigrina Mulsant, Pedostrangalia verticalis Germar, Brachyta balcanica Hampe şi Neodorcadion exornatum (Frivaldsky von Frivald), listate în anexele Directivei Habitate a Consiliului Europei 92/43 EEC referitoare la conservarea habitatelor naturale şi a speciilor de faună şi floră sălbatică şi în Ordonanța de Urgență 57/2007 privind regimul ariilor naturale protejate, conservarea habitatelor naturale, a florei şi faunei sălbatice.

\section{LITERATURE CITED}

BALACI, A., 2000 - Familia Cerambycidae (Coleoptera) din colecţia Secţiei de Ştiinţele Naturii a Muzeului Banatului Timişoara. Museum Arad. Armonii Naturale III: 418-426. (in Romanian)

BALOG, A., 1998 - Contribuţii la cunoașterea unor familii de coleoptere din zona Canaraua Fetii (Băneasa) (Dobrogea de Sud, Romania); Coleoptera: Scarabaeidae, Cerambycidae, Chrysomelidae, Buprestidae. Studii şi Cercetări (Ştiinţele Naturii), 4: 261-267. (in Romanian)

BEENEN, R., 2010 - Subfamily Galerucinae Latreille, 1802. Pp. 443-491. In: I. Löbl, A. Smetana (eds), Catalogue of Palaearctic Coleoptera, Chrysomeloidea, Volume 6, Apollo Books, Stenstrup, $924 \mathrm{pp}$.

BOROWIEC, L., L. SEKERKA, 2010 - Subfamily Cassidinae Gyllenhal, 1813. Pp. 368-390. In: I. Löbl, A. Smetana (eds), Catalogue of Palaearctic Coleoptera, Chrysomeloidea, Volume 6, Apollo Books, Stenstrup, 924 pp.

CRIŞAN, AL., 1993 - Date asupra Familiei Chrysomelidae (Coleoptera) în partea sudică a Deltei Dunării. Analele Știinţifice ale Institutului Delta Dunării, Tulcea, 2: 67-74. (in Romanian)

CRIŞAN, AL., 1994 - Noi date asupra familiei Chrysomelidae (Coleoptera) în Rezervaţia Biosferei "Delta Dunării“". Analele Ştiinţifice ale Institutului Delta Dunării, Tulcea, 3 (1): 159-166. (in Romanian)

CRIȘAN, AL., 1995 a - Cercetări faunistice asupra familiei Chrysomelidae (Coleoptera) în zona maritimă a Rezervaţiei Biosferei Delta Dunării. Analele Ştiinţifice ale Institutului Delta Dunării, Tulcea, 4 (1): 161-168. (in Romanian)

CRIŞAN, AL., 1995 b - Cercetări asupra familiei Chrysomelidae (Coleoptera) în zona Rezervaţiei Biosferei “Delta Dunării”, cu referire specială la Stylosomus tamaricis H.-S. și Cryptocephalus gamma H.-S. Buletinul de Informare al Societăţii Lepidopterologice Române, 6 (1-2): 145149. (in Romanian)

DASCĂLU, M. M., 2010 - New species of Cerambycidae (Coleoptera) for the Romanian fauna. Analele Ştiinţ̧ifice ale Universităţii “Al. I. Cuza” Iaşi, seria Biologie Animală, 56: 63-67.

DASCĂLU, M. M., L. FUSU, 2012 - Dorcadion axillare Küster, 1847 - (Coleoptera, Cerambycidae): distribution, morphometrics, karyotype and description of a new subspecies from Romania. Zootaxa, 3322: 35-48.

DÖBERL, M., 2010 - Subfamily Alticinae Newman, 1835. Pp. 491-563. In: I. Löbl, A. Smetana (eds), Catalogue of Palaearctic Coleoptera, Chrysomeloidea, Volume 6, Apollo Books, Stenstrup, $924 \mathrm{pp}$.

FLECK, ED., 1905 a - Die Coleopteren Rumänien. Buletinul Societăţii de Ştiințe Bucureşti, 14 (5): 491-570.

FLECK, ED., 1905 b - Die Coleopteren Rumänien. Buletinul Societăţii de Ştiinţe Bucureşti, 14 (6): 680-735. 
GOMOIU, M. T., M. SKOLKA, 1998 - Evaluation of marin and coastal biological diversity at the Romanian littoral - a workbook for the Black Sea ecological diversity. Analele Universităţii "Ovidius" Constanţa, Seria Biologie - Ecologie, supl.

GRUEV, B. A., 1983 - New localities of some leaf beetles (Chrysomelidae, Coleoptera) on the Balkan Peninsula. Travaux Scientifiques d'Université de Plovdiv, Biologie, 21 (4): 21-30.

GRUEV, B. A., 2001 - Actualized checklist of Alticinae (Coleoptera: Chrysomelidae) in the Balkan Peninsula. Travaux Scientifiques d'Université de Plovdiv, Animalia, 37 (6): 9-28.

GRUEV, B .A., 2002 - New distributional data about some leaf beetles (Coleoptera, Chrysomelidae: Eumolpinae, Chrysomelinae, Alticinae, Hispinae, Cassidinae) in the Balkan Peninsula. Travaux Scientifiques d'Université de Plovdiv, Animalia, 38 (6): 17-34.

GRUEV, B. A., 2005 - A comparative list of the leaf beetles of the Balkan countries (Coleoptera: Chrysomelidae). Animalia, 41: 23-46.

GRUEV, B., O. MERKL, K. VIG, 1993 - Geographical distribution of Alticinae (Coleoptera, Chrysomelidae) in Romania. Annales Historico-Naturales Musei Nationalis Hungarici, 85: $75-132$.

HOINIC, C., 1994 - A review of the species of Macroplea Samouelle (Coleoptera: Chrysomelidae) in Romania. Travaux du Muséum d'Histoire Naturelle "Grigore Antipa", 34: 17-30.

HOINIC, C., 1995 - Stylosomus flavus (Coleoptera: Chrysomelidae) a new species in Romanian fauna. Travaux du Muséum d'Histoire Naturelle "Grigore Antipa", 35: 147-152.

HOSKOVEC, M., M. REJZEK, 2009 - Longhorn Beetles (Cerambycidae) of the West Palaearctic Region. Available online at: http:/www.cerambyx.uochb.cz.

HURMUZACHI, C., 1904 - Troisième catalogue des coléoptères recoltés par les membres de la Societé des Naturalistes de Roumanie. Buletinul Societăţii de Sciinţe din Bucuresci, 3 (1-2): $52-65$.

IENIŞTEA, M. AL., 1968 - Ord. Coleoptera (pars). In: Entomofaune de l'ile de Letea (Delta du Danube). Travaux du Muséum d'Histoire Naturelle "Grigore Antipa", 9: 97-114.

IENIŞTEA, M. AL., 1974 - Contributions à la connaissance des Coléoptères du Delta du Danube (Le "grind" Caraorman). Travaux du Muséum d'Histoire Naturelle "Grigore Antipa", 14: 239-249.

IENIŞTEA, M. AL., ŞT. NEGRU, 1956 - Specii de coleoptere noi pentru fauna României. Comunicările Academiei Române, 6 (8): 995-997. (in Romanian)

IONESCU-KONNERTH, A., 1963 - Halticinae recorded from Romania till 1961. Travaux du Muséum d'Histoire Naturelle "Grigore Antipa", 4: 251-267.

JAQUET, M., 1898 a - Faune de la Roumanie. Insectes récoltés par Mr. le Dr. M. Jaquet en 1897 et détérminés par Mr. le Dr. E. Poncy, entomologiste à Genève. Buletinul Societăţii de Ştiinţe Bucuresci, 7 (1): 52-56.

JAQUET, M., 1898 b - Faune de la Roumanie. Coléoptères récoltés par M. Jaquet en 1897 et détérminés par Mr. E. Poncy, entomologiste à Genève. Buletinul Societăţii de Ştiinţe Bucuresci, 7 (5): 493-485.

JAQUET, M., 1899 - Faune de la Roumanie. Coléoptères récoltés par Mr. le Dr. M. Jaquet en 1898 et détérminés par Mr. le Dr. E. Poncy, entomologiste à Genève. Buletinul Societăţii de Ştiinţe Bucuresci, 8 (1-2): 121-214.

JAQUET, M., 1900 a - Faune de la Roumanie. Insectes récoltés par Mr. le Dr. M. Jaquet et détérminés par Mr. le Dr. E. Poncy, entomologiste à Genève. Buletinul Societăţii de Ştiinţe Bucuresci, 9 (4): 392-400.

JAQUET, M., 1900 b - Faune de la Roumanie. Coléoptères récoltés en 1899 par M. Le Dr. Jaquet et déterminés par M. E. Poncy à Genève. Buletinul Societăţii de Ştiinţe Bucuresci, 9 (6): 754762.

JAQUET, M., 1901 - Faune de la Roumanie. Insectes récolté par Mr. le Dr. M. Jaquet en 1899 et détérminés par Mr. le Dr. E. Poncy, entomologiste à Genève. Buletinul Societăţii de Ştiinţe Bucuresci, 10 (5): 483-497.

JAQUET, M., 1903 - Faune de la Roumanie. Coléoptères récoltés par Mr. le Dr. M. Jaquet en 1899 et détérminés par Mr. le Dr. E. Poncy, entomologiste à Genève. Buletinul Societăţii de Ştiinţe Bucuresci, 12 (1-2): 88-96.

KIPPENBERG, H., 2010 - Subfamily Chrysomelinae Latreille, 1802. Pp. 390-443. In: I. Löbl, A. Smetana (eds), Catalogue of Palaearctic Coleoptera, Chrysomeloidea, Volume 6, Apollo Books, Stenstrup, $924 \mathrm{pp}$.

KNECHTEL, W., S. PANIN, 1944 - Oekologisch - Zoogeographisches studium an Coleopteren des Rumänischen Faunengebietes. Académie Roumaine. Etudes et Recherches, 15: 1-219. 
LOPATIN, I., A. SMETANA, M. SCHÖLLER, I. LÖBL, 2010 - Tribe Cryptocehalini, Gyllenhal, 1813. Pp. 580-617. In: I. Löbl, A. Smetana (eds), Catalogue of Palaearctic Coleoptera, Chrysomeloidea, Volume 6, Apollo Books, Stenstrup, 924 pp.

LÖBL, I., A. SMETANA (eds.), 2010 - Catalogue of Palaearctic Coleoptera. Chrysomeloidea, Volume 6, Apollo Books, Stenstrup, 924 pp.

MAICAN, S., 2006 a - Clytrins (Coleoptera: Chrysomelidae: Clytrinae) from the collections of the "Grigore Antipa" National Museum of Natural History (Bucharest). Travaux du Muséum National d'Histoire Naturelle "Grigore Antipa", 49: 239-257.

MAICAN, S., 2006 b - Chrysomelids (Coleoptera: Chrysomelidae) from the sylvosteppe forests of Romania. Romanian Journal of Biology-Zoology, Bucharest. Edit. Academiei Române, 51 (1-2): 13-25.

MONTANDON, A. L., 1887 - Excursions en Dobroudja. Bulletin de la Société d'Études Scientifiques d'Angers (Nouvelle série), 16: 31-64.

MONTANDON, A. L., 1906 - Notes sur la faune entomologique de la Roumanie (Coleoptera). Buletinul Societății de Ştiinţe Bucuresci, 15 (1-2): 30-80.

MONTANDON, A. L., 1908 - Notes sur la faune entomologique de la Roumanie. Additions au Catalogue des Coléoptères. Buletinul Societăţii de Ştiinţe Bucuresci, 17 (1-2): 67-122.

MOSEYKO, A. G., E. SPRECHER-UEBERSAX, 2010 - Subfamily Eumolpinae Hope, 1840. Pp. 619643. In: I. Löbl, A. Smetana (eds), Catalogue of Palaearctic Coleoptera, Chrysomeloidea, Volume 6, Apollo Books, Stenstrup, 924 pp.

NAGY, C., 1972 - Contributions à l'étude des insectes du supralittoral du secteur roumain de la Mer Noire. Cercetări marine I.R.C.M., 3: 171-189.

NEGRU, ŞT., 1957 - Contribuţiune la cunoaşterea faunei coleopterologice a Mangaliei şi împrejurimilor ei (I). Analele Universităţii C. I. Parhon-Bucureşti, Seria Ştiinţele Naturii, 16: 117-138. (in Romanian)

NEGRU, ST., 1965 - Sur un nouvel ennemi du fustet (Cotinus coggygria Scop.). (Contribution à l'étude de l'entomofaune de la Dobrogea). Travaux du Muséum d'Histoire Naturelle "Grigore Antipa", 5: 501-504.

NEGRU, ŞT., 1968 a - Ord. Coleoptera (pars). In: Entomofaune de l'ile de Letea (Delta du Danube). Travaux du Muséum d'Histoire Naturelle "Grigore Antipa", 9: 81-93.

NEGRU, ŞT., 1968 b - Phymatodes fasciatum (Coleoptera: Cerambycidae), agent nuisible de la vigne sauvage dans le Delta du Danube. Travaux du Muséum d'Histoire Naturelle "Grigore Antipa", 9: 447-452.

NEGRU, ŞT., A. ROŞCA, 1967 - Ord. Coleoptera. In: L'entomofaune des forêts du sud de la Dobrogea. Travaux du Muséum d'Histoire Naturelle "Grigore Antipa", Bucarest, 7: 119-148.

NITZU, E., 2001 - Edaphic and subterranean Coleoptera from the Dobrogea carstic areas (Romania). A zoogeographic approach. Mitteilungen aus dem Hamburgischen Zoologischen Museum und Institut, Hamburg, 98: 131-169.

PANIN, S., 1944 - Les Chrysomela de la Roumanie. Bulletin de la Section Scientifique de l'Académie Roumaine, 26 (9): 601-625.

PANIN, S., N. SĂVULESCU, 1961 - Coleoptera: Cerambycidae. In: Fauna României, 10 (5): 1-523. Edit. Academiei Române. (in Romanian)

PETRI, K., 1912 - Siebenbürgens Käferfauna auf Grund ihrer Erforschung bis zum Jahre 1911. Verhandlungen und Mitteilungen des Siebenbürgischen Vereins fur Naturwissenschaften zu Hermannstadt: 1-376.

REGAliN, R., L. N. MEDVEDEV, 2010 - Tribe Cytrini Kirby, 1837. Pp. 564-580. In: I. Löbl, A. Smetana (eds), Catalogue of Palaearctic Coleoptera, Chrysomeloidea, Volume 6, Apollo Books, Stenstrup, 924 pp.

ROŞCA, A., 1973 - Contributions à la connaissance du genre Cryptocephalus Fourcr. (Coleoptera, Chrysomelidae) en Roumanie (I). Travaux du Muséum d'Histoire Naturelle "Grigore Antipa", 13: 143-154.

ROŞCA, A., 1974 - Contributions à la connaissance du genre Chrysomela L. (Chrysomelidae, Coleoptera) en Roumanie. Travaux du Muséum d'Histoire Naturelle "Grigore Antipa", 14: 251-259.

ROŞCA, A., 1976 - Ord. Coleoptera (pars). In: L'entomofaune du Nord de la Dobrogea, la Zone MăcinTulcea-Niculiţel. Travaux du Muséum d'Histoire Naturelle "Grigore Antipa", 17: 145-152.

SAMA, G., 2005 - Fauna Europaea: Cerambycidae. In: P. Audisio, 2005 - Fauna Europaea: Coleoptera 2. Fauna Europaea version 1.2. Available online at: http://www.faunaeur.org.

SAMA, G., LÖBL, I., 2010 - Cerambycidae - Western Palaearctic taxa, eastward to Afghanistan, excluding Oman and Yemen and the countries of the former Soviet Union. P. 186. In: I. Löbl, 
A. Smetana (eds), Catalogue of Palaearctic Coleoptera, Chrysomeloidea, Volume 6, Apollo Books, Stenstrup, 924 pp.

SAMA, G., M. M. DASCALU, C. PESARINI, 2010 - Description of Dorcadion gashtarovi n. sp. (Coleoptera, Cerambycidae) from Romania and Bulgaria with review of the closely related species. North-Western Journal of Zoology, 6 (2): 286-293.

SĂVULESCU, 1969 - Câteva observaţii ecologice asupra speciilor din România ale genului Cerambyx L. (Col. Cerambycidae). Comunicări de Zoologie. Prima Consfătuire Naţională de Entomologie, 2: 281-290. (in Romanian)

SĂVULESCU, N., 1985 - Prezențe şi absențe enigmatice în entomofauna Deltei Dunării. Studii şi Comunicări de Entomologie, Delta Dunării II, Tulcea: 147-157. (in Romanian)

SĂVULESCU, N., A. POPESCU-GORJ, 1964 - Pădurile din sud-vestul Dobrogei, monumente puţin cunoscute ale naturii. Ocrotirea Naturii, 8 (2): 257 - 276. (in Romanian)

SCHMITT, M., 2010 - Subfamily Criocerinae Latreille, 1804. Pp. 359-368. In: I. Löbl, A. Smetana (eds), Catalogue of Palaearctic Coleoptera, Chrysomeloidea, Volume 6, Apollo Books, Stenstrup, $924 \mathrm{pp}$.

SERAFIM, R., 1993,- Contribution à la connaissance des Coléoptères Cerambycides (Coleoptera, Cerambycidae) du Delta du Danube et du complex lagunaire Razelm (Razim) (Roumanie). Travaux du Muséum d'Histoire Naturelle "Grigore Antipa", 33: 235-246.

SERAFIM, R., 2005 - Catalogue of the Palaearctic species of Prioninae and Lepturinae (Coleoptera: Cerambycidae) from patrimony of "Grigore Antipa" National Museum of Natural History, Bucharest (Part I). Travaux du Muséum National d'Histoire Naturelle "Grigore Antipa”, 48: 103-117.

SERAFIM, R., 2006 - The Catalogue of the Palaearctic species of Lepturinae (Coleoptera: Cerambycidae) from the patrimony of "Grigore Antipa" National Museum of Natural History, Bucharest (Part II). Travaux du Muséum National d'Histoire Naturelle "Grigore Antipa", 49: 203-238.

SERAFIM, R., 2007 - The Catalogue of the Palaearctic species of Spondylidinae (Coleoptera: Cerambycidae) from the patrimony of "Grigore Antipa" National Museum of Natural History (Bucharest) (Part III). Travaux du Muséum National d'Histoire Naturelle "Grigore Antipa", 50: 221-230.

SERAFIM, R., 2009 - The Catalogue of the Palaearctic species of Necydalinae and Cerambycinae (Coleoptera: Cerambycidae) from the patrimony of "Grigore Antipa" National Museum of Natural History, Bucharest (Bucureşti) (Part IV). Travaux du Muséum National d'Histoire Naturelle "Grigore Antipa", 52: 263-292.

SERAFIM, R., 2010 - The Catalogue of the Palaearctic species of Lamiinae (Coleoptera: Cerambycidae) from the patrimony of "Grigore Antipa" National Museum of Natural History (Bucharest) (Part V). Travaux du Muséum National d'Histoire Naturelle "Grigore Antipa", 53: 235-272.

SERAFIM R., M. APETREI, 1996 - Coleoptere Coccinellidae şi Cerambycidae din colecţia Muzeului de Ştiinţele Naturii din Piatra Neamţ. Complexul Muzeal Judeţean Neamţ, Studii şi Cercetări, 8: 431- 453. (in Romanian)

SERAFIM, R., S. MAICAN, 2004 - Contributions to the knowledge of the Coleopterans from the littoral of the Black Sea (Romania). Travaux du Muséum National d'Histoire Naturelle "Grigore Antipa", 47: 169-210.

SERAFIM, R., S. MAICAN, 2011 - Catalogue of Cerambycidae, Megalopodidae and Chrysomelidae (Coleoptera: Chrysomeloidea) recently entered in the patrimony of "Grigore Antipa" National Museum of Natural History (Bucharest). Igor Ceianu collection. Travaux du Muséum National d'Histoire Naturelle "Grigore Antipa", 54 (2): 425-460.

SERAFIM, R., C. CHIMIŞLIU, N. G. LILA, 2004 - Catalogul cerambycidelor (Coleoptera, Cerambycoidea, Cerambycidae) din patrimoniul Muzeului Olteniei, Craiova. Oltenia, Studii şi Comunicări, 20: 189-198. (in Romanian)

SILFVERBERG, H., 2010 - Family Orsodacnidae C. H. Thomson, 1859. P. 337. In: I. Löbl, A. Smetana (eds), Catalogue of Palaearctic Coleoptera, Chrysomeloidea, Volume 6, Apollo Books, Stenstrup, 924 pp.

SKOLKA, M., M. FĂGĂRAŞ, G. M. PARASCHIV, 2005 - Biodiversitatea Dobrogei. Ovidius University Press, Constanţa, 396 pp. (in Romanian)

SKOLKA, M., I. CARP, S. GRIGORE, C. STANCIU, 2006-2007 - Evaluarea biodiversităţii populaţ̧iilor de insecte din Parcul Naţional Munţii Măcinului. Available at: http://www. parcmacin.ro/app/webroot/files/insecte.pdf (in Romanian)

TATOLE, V., A. IFTIME, M. STAN, E. I. IORGU, I. IORGU, V. OŢEL, 2009 - Speciile de animale Natura 2000 din România: 1-174. (in Romanian) 
TĂUŞAN, I., C. BUCŞA, 2010 - Genus Cerambyx L., 1758 (Coleoptera: Cerambycidae) in the Natural History Museum Collections of Sibiu (Romania). Brukenthal Acta Musei, 3: 607-612.

TOGĂNEL, F., 2004 - Cerambycide (Coleoptera: Cerambycoidea) din colecţia Muzeului de Ştiinţele Naturii din Târgu Mureş, Muzeul Olteniei Craiova. Oltenia, Studii şi Comunicări, Ştiiinţele Naturii, 20: 203-213. (in Romanian)

VASILIU-OROMULU, L., S. MAICAN, I. TELCEAN, 2004 - The conservation of the faunistical biodiversity, the preservation of endagered species from endagered habitats. Proceedengs of the Institute of Biology, 6: 161-166.

VIG, K., 1997 - Leaf beetle collection of the Mátra Museum, Gyöngyös, Hungary (Coleoptera, Chrysomelidae sensu lato). Folia Historico Naturalia Musei Matraensis, 22: 175-201.

WARCHAŁOWSKI, A., 2003 - Chrysomelidae. The leaf beetles of Europe and the Mediterranean area. Natura Optima dux. Foundation, Warszawa, 600 pp.

*** European Commission, 1992 - Council Directive 92/43/EEC of 21 May 1992 on the conservation of natural habitats and of wild fauna and flora (online). Official Journal L 206, 22/07/1992 P. 0007-0050. Consolidated version 01.01.2007. Available at: http://ec.europa.eu/ environment/ nature/legislation/habitatsdirective/index_en.htm.

*** Ordonanţa de urgenţă nr. 57 din 20/06/2007 privind regimul ariilor naturale protejate, conservarea habitatelor naturale, a florei şi faunei sălbatice, Monitorul Oficial, Partea I, nr. 442 din 29/06/2007 (in Romanian)

Received: May 15, 2012

Accepted: July 24, 2012

\author{
Rodica Serafim \\ "Grigore Antipa" National Museum of Natural History \\ Şos. Kiseleff 1, 011341 Bucharest 2, Romania \\ e-mail: serafim@antipa.ro \\ Sanda Maican \\ Institute of Biology Bucharest of Romanian Academy, \\ 296 Splaiul Independenţei, 060031 Bucharest, \\ P.O. Box 56-53, Romania \\ e-mail: sanda.maican@ibiol.ro
}

